\title{
Detecting a stochastic background of gravitational radiation: Signal processing strategies and sensitivities
}

\author{
Bruce Allen* and Joseph D. Romano ${ }^{\dagger}$ \\ Department of Physics, University of Wisconsin-Milwaukee, Milwaukee, Wisconsin 53201
}

(Received 28 October 1997; published 31 March 1999)

\begin{abstract}
We analyze the signal processing required for the optimal detection of a stochastic background of gravitational radiation using laser interferometric detectors. Starting with basic assumptions about the statistical properties of a stochastic gravity-wave background, we derive expressions for the optimal filter function and signal-to-noise ratio for the cross-correlation of the outputs of two gravity-wave detectors. Sensitivity levels required for detection are then calculated. Issues related to (i) calculating the signal-to-noise ratio for arbitrarily large stochastic backgrounds, (ii) performing the data analysis in the presence of nonstationary detector noise, (iii) combining data from multiple detector pairs to increase the sensitivity of a stochastic background search, (iv) correlating the outputs of 4 or more detectors, and (v) allowing for the possibility of correlated noise in the outputs of two detectors are discussed. We briefly describe a computer simulation that was used to "experimentally" verify the theoretical calculations derived in the paper, and which mimics the generation and detection of a simulated stochastic gravity-wave signal in the presence of simulated detector noise. Numerous graphs and tables of numerical data for the five major interferometers (LIGO-WA, LIGO-LA, VIRGO, GEO600, and TAMA-300) are also given. This information consists of graphs of the noise power spectra, overlap reduction functions, and optimal filter functions; also included are tables of the signal-to-noise ratios and sensitivity levels for cross-correlation measurements between different detector pairs. The treatment given in this paper should be accessible to both theorists involved in data analysis and experimentalists involved in detector design and data acquisition. [S0556-2821(99)02708-3]

PACS number(s): 04.80.Nn, 04.30.Db, 07.05.Kf, 95.55.Ym
\end{abstract}

\section{INTRODUCTION}

The design and construction of a number of new and more sensitive detectors of gravitational radiation is currently underway. These include the two Laser Interferometric Gravitational Wave Observatory (LIGO) detectors being built in Hanford, WA and Livingston, LA by a joint Caltech-MIT collaboration [1], the VIRGO detector being built near Pisa, Italy by an Italian-French collaboration [2], the GEO-600 detector being built in Hanover, Germany by an AngloGerman collaboration [3], and the TAMA-300 detector being built near Tokyo, Japan [4]. There are also several resonant bar detectors currently in operation, and several more refined bar and interferometric detectors presently in the planning and proposal stages.

The operation of these detectors will have a major impact on the field of gravitational physics. For the first time, there will be a significant amount of experimental data to be analyzed, and the "ivory tower" relativists will be forced to interact with a broad range of experimenters and data analysts to extract the interesting physics from the data stream. "Known', sources such as coalescing neutron star (or black hole) binaries, pulsars, supernovae, and other periodic and transient (or burst) sources should all be observable with

\footnotetext{
*Email address: ballen@dirac.phys.uwm.edu

${ }^{\dagger}$ Permanent address: Department of Physical Sciences, University of Texas at Brownsville, Brownsville, Texas 78521 (Email address: jromano@utb1.utb.edu)
}

gravity-wave detectors. One might also be able to detect a faint stochastic background of gravitational radiation, produced very shortly after the big bang. These detections may happen soon after the detectors go "on-line" or they may require a decade of further work to increase the sensitivity of the instruments. But it is fairly safe to say that eventually, when their sensitivity passes some threshold value, the gravity-wave detectors will find sources. Even more exciting is the prospect that the detectors will discover new sources of gravitational radiation-sources which are different from those mentioned above, and which we had not expected to find. It promises to be an exciting time.

The subject of this paper is a stochastic (i.e., random) background of gravitational radiation, first studied in detail by Michelson [5], Christensen [6], and Flanagan [7]. Roughly speaking, it is the type of gravitational radiation produced by an extremely large number of weak, independent, and unresolved gravity-wave sources. The radiation is stochastic in the sense that it can be characterized only statistically. As mentioned above, a stochastic background of gravitational radiation might be the result of processes that took place very shortly after the big bang. But since we know very little about the state of the universe at that time, it is impossible to say with any certainty. A stochastic background of gravitational radiation might also arise from more recent processes (e.g., radiation from many unresolved binary star systems), and this more recent contribution might overwhelm the parts of the background that contain information about the state of the early universe. In any case, the properties of the radiation will be very dependent upon the source. For example, one would expect a stochastic back- 
ground of cosmological origin to be highly isotropic, whereas that produced by white dwarf binaries in our own galaxy would be highly anisotropic. We will just have to wait and see what the detectors reveal before we can decide between these two possibilities.

This paper will focus on issues related to the detection of a stochastic background of gravitational radiation. (We will not talk much about possible sources.) We give a complete and comprehensive treatment of the problem of detecting a stochastic background, which should be accessible to both theorists involved in the data analysis and experimentalists involved in detector design and data acquisition.

The outline of the paper is as follows:

In Sec. II, we begin by describing the properties of a stochastic background of gravitational radiation-its spectrum, statistical assumptions, and current observational constraints.

In Sec. III, we describe how one can correlate the outputs of two gravity-wave detectors to detect (or put an upper limit on) a stochastic gravity-wave signal. Section III B includes a detailed derivation of the overlap reduction function that covers the case where the two arms of a detector are not perpendicular (e.g., GEO-600) and corrects a typographical error that appears in the literature. Section III C includes a rigorous derivation of the optimal signal processing strategy. Most of the material in Secs. II and III has already appeared in the literature. Interested readers should see Ref. [8] for more details, if desired.

In Sec. IV, we ask the following questions: (i) How do we decide, from the experimental data, if we've detected a stochastic gravity-wave signal? (ii) Assuming that a stochastic gravity-wave signal is present, how do we estimate its strength? (iii) Assuming that a stochastic gravity-wave signal is present, what is the minimum value of $\Omega_{0}$ required to detect it $95 \%$ of the time? This leads to a discussion of signal detection, parameter estimation, and sensitivity levels for stochastic background searches, adopting a frequentist point of view. The calculation of $\Omega_{0}^{95 \%, 5 \%}$ in Sec. IV D corrects an error that has appeared in the literature.

In Sec. V, we return to the problem of detection by addressing a series of subtle issues initially ignored in Sec. III. These include (i) calculating the signal-to-noise ratio for arbitrarily large stochastic backgrounds, (ii) performing the data analysis in the presence of nonstationary detector noise, (iii) combining data from multiple detector pairs to increase the sensitivity of a stochastic background search, (iv) correlating the outputs of 4 or more detectors, and (v) allowing for the possibility of correlated noise in the outputs of two detectors. The material presented in these sections extends the initial treatment of these issues given, for example, in Refs. $[6,7]$.

Section VI consists of a series of graphs and tables of numerical data for the five major interferometers (LIGOWA, LIGO-LA, VIRGO, GEO-600, TAMA-300). The noise power spectra, overlap reduction functions, optimal filter functions, signal-to-noise ratios, and sensitivity levels for cross-correlation measurements between different detector pairs (not just LIGO) are given. This information allows us to determine the optimal way of combining data from mul- tiple detector pairs when searching for a stochastic background of gravitational radiation. Section VI A also includes a graph of the "enhanced" LIGO detector noise curves, which track the projected performance of the LIGO detector design over the next decade.

In Sec. VII, we describe a computer simulation that mimics the generation and detection of a simulated stochastic gravity-wave signal in the presence of simulated detector noise. The simulation was used to verify some of the theoretical calculations derived in the previous sections.

Section VIII concludes the paper with a brief summary and lists some topics for future work.

Note that throughout the paper, we use $c$ to denote the speed of light and $G$ to denote Newton's gravitational constant $\left(c=2.998 \times 10^{10} \mathrm{~cm} / \mathrm{sec}\right.$ and $G=6.673 \times 10^{-8}$ $\left.\mathrm{cm}^{3} / \mathrm{g} \mathrm{sec}^{2}\right)$.

\section{STOCHASTIC BACKGROUND: PROPERTIES}

\section{A. Spectrum}

A stochastic background of gravitational radiation is a random gravity-wave signal produced by a large number of weak, independent, and unresolved gravity-wave sources. In many ways it is analogous to the cosmic microwave background radiation (CMBR) [9], which is a stochastic background of electromagnetic radiation. As with the CMBR, it is useful to characterize the spectral properties of the gravitational background by specifying how the energy is distributed in frequency. Explicitly, one introduces the dimensionless quantity

$$
\Omega_{\mathrm{gw}}(f):=\frac{1}{\rho_{\text {critical }}} \frac{d \rho_{\mathrm{gw}}}{d \ln f},
$$

where $d \rho_{\mathrm{gw}}$ is the energy density of the gravitational radiation contained in the frequency range $f$ to $f+d f$, and $\rho_{\text {critical }}$ is the critical energy density required (today) to close the universe:

$$
\rho_{\text {critical }}=\frac{3 c^{2} H_{0}^{2}}{8 \pi G} \approx 1.6 \times 10^{-8} h_{100}^{2} \frac{\mathrm{ergs}}{\mathrm{cm}^{3}} .
$$

$H_{0}$ is the Hubble expansion rate (today),

$$
\begin{aligned}
H_{0} & =h_{100} \times 100 \frac{\mathrm{km}}{\mathrm{sec} \mathrm{Mpc}}=3.2 \times 10^{-18} h_{100} \frac{1}{\mathrm{sec}} \\
& =1.1 \times 10^{-28} \operatorname{ch}_{100} \frac{1}{\mathrm{~cm}},
\end{aligned}
$$

and $h_{100}$ is a dimensionless factor, included to account for the different values of $H_{0}$ that are quoted in the literature. ${ }^{1}$ It is this dimensionless function of frequency, $\Omega_{\mathrm{gw}}(f)$, that we will use to describe the spectrum of a stochastic background of gravitational radiation. It follows directly from the above definitions that $\Omega_{\mathrm{gw}}(f) h_{100}^{2}$ is independent of the actual

\footnotetext{
${ }^{1} h_{100}$ almost certainly lies within the range $1 / 2<h_{100}<1$.
} 
Hubble expansion rate. For this reason, we will often focus attention on this quantity, rather than on $\Omega_{\mathrm{gw}}(f)$ alone.

Two remarks are in order:

(i) There appears to be some confusion about $\Omega_{\mathrm{gw}}(f)$ in the literature. Some authors assume that $\Omega_{\mathrm{gw}}(f)$ is constant-i.e., independent of frequency. Although this is true for some cosmological models, it is not true for all of them. The important point is that any spectrum of gravitational radiation can be described by an appropriate $\Omega_{\mathrm{gw}}(f)$. With the correct dependence on frequency, $\Omega_{\mathrm{gw}}(f)$ can describe a flat spectrum, a blackbody spectrum, or any other distribution of energy with frequency.

(ii) $\Omega_{\mathrm{gw}}(f)$ is the ratio of the stochastic gravity-wave energy density contained in a bandwidth $\Delta f=f$ to the total energy density required to close the universe. For the CMBR, one can define an analogous quantity:

$$
\Omega_{\mathrm{em}}(f):=\frac{1}{\rho_{\text {critical }}} \frac{d \rho_{\mathrm{em}}}{d \ln f} .
$$

Since the $2.73 \mathrm{~K}$ blackbody spectrum has a peak value of $\Omega_{\mathrm{em}}(f) h_{100}^{2} \approx 10^{-5}$ at $f=10^{12} \mathrm{~Hz}$, the CMBR contains (in the vicinity of $10^{12} \mathrm{~Hz}$ ) approximately $10^{-5}$ of the total energy density required to close the universe. A similar interpretation applies to $\Omega_{\mathrm{gw}}(f)$.

\section{B. Statistical assumptions}

The spectrum $\Omega_{\mathrm{gw}}(f)$ completely specifies the stochastic background of gravitational radiation provided we make enough additional assumptions. We will assume that the stochastic background is (i) isotropic, (ii) unpolarized, (iii) stationary, and (iv) Gaussian. Since these properties might not hold in general, it is worthwhile to consider each one of them in turn.

(i) Since it is now well established that the CMBR is highly isotropic [9], it is not unreasonable to assume that a stochastic background of gravitational radiation is also isotropic. But this assumption might not be true. For example, as mentioned in Sec. I, if the dominant source of the stochastic gravity-wave background is a large number of unresolved white dwarf binary star systems within our own galaxy, then the stochastic background will have a distinctly anisotropic distribution, which forms a "band in the sky" distributed roughly in the same way as the Milky Way galaxy. It is also possible for a stochastic gravity-wave background of cosmological origin to be anisotropic, although one would then have to explain why the CMBR is isotropic but the gravitywave background is not. In either case, such anisotropies can be searched for in the data stream. (See Ref. [10] for details.)

(ii) The second assumption is that the stochastic gravitywave background is unpolarized. This means that the gravitational radiation incident on a detector has statistically equivalent "plus" and "cross" polarization components. We see no strong reason why this should not be the case.

(iii) The assumption that the stochastic background is stationary (i.e., that all statistical quantities depend only upon the difference between times, and not on the choice of time origin) is almost certainly justified. This is because the age of the universe is roughly 20 orders of magnitude larger than the characteristic period of the waves that LIGO, VIRGO, etc. can detect, and 9 orders of magnitude larger than the longest realistic observation times. It seems very unlikely that a stochastic background of gravitational radiation would have statistical properties that vary over either of these time scales. But unlike the stochastic gravity-wave background, the noise intrinsic to the detectors will change over the course of the observation times. This poses a problem for the data analysis, which we initially ignore in Sec. III. We return to this problem in Sec. V B where we discuss nonstationary detector noise.

(iv) The final assumption is that the stochastic gravitywave background is a Gaussian random process. This means that the joint probability density function of the gravitational strains $h_{i}\left(t_{i}\right), h_{j}\left(t_{j}\right), \ldots$ in detectors $i, j, \ldots$ is a multivariate Gaussian (i.e., normal) distribution. In this case, the mean values $\left\langle h_{i}(t)\right\rangle$ and the second-order moments $\left\langle h_{i}\left(t_{i}\right) h_{j}\left(t_{j}\right)\right\rangle$ completely specify the statistical properties of the signal. For many early-universe processes, or even for more recent sources of a gravity-wave background, this is a reasonable assumption. It can be justified by the central limit theorem, which says that the sum of a large number of statistically independent random variables is a Gaussian random variable, independent of the probability distributions of the original variables. This will be the case for the stochastic background if it is the sum of gravity-wave signals produced by a large number of independent gravity-wave sources. This assumption will not be true, however, if the stochastic background is the sum of the radiation produced, e.g., by only a few unresolved binary star systems radiating in a given frequency interval at any instant of time. (See, e.g., Ref. [11].)

The above four properties form the basis for the statistical analysis that we will give in the following sections. We will assume that they hold throughout, unless we explicitly state otherwise.

\section{Expectation value}

Using the definition of the spectrum $\Omega_{\mathrm{gw}}(f)$ and the statistical assumptions described in the previous subsection, we can derive a useful result for the expectation value of the Fourier amplitudes of a stochastic background of gravitational radiation. This result will be needed in Sec. III when we discuss signal detection and optimal filtering.

The starting point of the derivation is a plane wave expansion for the gravitational metric perturbations in a transverse, traceless gauge:

$$
h_{a b}(t, \vec{x})=\sum_{A} \int_{-\infty}^{\infty} d f \int_{S^{2}} d \hat{\Omega} h_{A}(f, \hat{\Omega}) e^{i 2 \pi f(t-\hat{\Omega} \cdot \vec{x} / c)} e_{a b}^{A}(\hat{\Omega}) .
$$

Here $\hat{\Omega}$ is a unit vector specifying a direction on the twosphere, with wave vector $\vec{k}:=2 \pi f \hat{\Omega} / c$. Also, $e_{a b}^{A}(\hat{\Omega})$ are the spin-2 polarization tensors for the "plus" and "cross" polarization states $A=+, \times$. Explicitly,

$$
e_{a b}^{+}(\hat{\Omega})=\hat{m}_{a} \hat{m}_{b}-\hat{n}_{a} \hat{n}_{b},
$$




$$
e_{a b}^{\times}(\hat{\Omega})=\hat{m}_{a} \hat{n}_{b}+\hat{n}_{a} \hat{m}_{b}
$$

where

$$
\begin{aligned}
& \hat{\Omega}=\cos \phi \sin \theta \hat{x}+\sin \phi \sin \theta \hat{y}+\cos \theta \hat{z}, \\
& \hat{m}=\sin \phi \hat{x}-\cos \phi \hat{y}, \\
& \hat{n}=\cos \phi \cos \theta \hat{x}+\sin \phi \cos \theta \hat{y}-\sin \theta \hat{z},
\end{aligned}
$$

and $(\theta, \phi)$ are the standard polar and azimuthal angles on the two-sphere. The Fourier amplitudes $h_{A}(f, \hat{\Omega})$ are arbitrary complex functions that satisfy $h_{A}(-f, \hat{\Omega})=h_{A}^{*}(f, \hat{\Omega})$, where * denotes complex conjugation. This last relation follows as a consequence of the reality of $h_{a b}(t, \vec{x})$.

The assumptions that the stochastic background is isotropic, unpolarized, and stationary imply that the expectation value (i.e., ensemble average) of the Fourier amplitudes $h_{A}(f, \hat{\Omega})$ satisfies

$$
\left\langle h_{A}^{*}(f, \hat{\Omega}) h_{A^{\prime}}\left(f^{\prime}, \hat{\Omega}^{\prime}\right)\right\rangle=\delta^{2}\left(\hat{\Omega}, \hat{\Omega}^{\prime}\right) \delta_{A A^{\prime}} \delta\left(f-f^{\prime}\right) H(f),
$$

where $\delta^{2}\left(\hat{\Omega}, \hat{\Omega}^{\prime}\right):=\delta\left(\phi-\phi^{\prime}\right) \delta\left(\cos \theta-\cos \theta^{\prime}\right)$ is the covariant Dirac delta function on the two-sphere, and $H(f)$ is a real, non-negative function, satisfying $H(f)=H(-f) .^{2}$ If we further assume that the stochastic background has zero mean, then

$$
\left\langle h_{A}(f, \hat{\Omega})\right\rangle=0 .
$$

Finally, since we are assuming that the stochastic background is Gaussian, the expectation values (2.11) and (2.12) completely specify its statistical properties.

$H(f)$ is related to the spectrum $\Omega_{\mathrm{gw}}(f)$ of the stochastic gravity-wave background. This follows from the expression

$$
\rho_{\mathrm{gw}}=\frac{c^{2}}{32 \pi G}\left\langle\dot{h}_{a b}(t, \vec{x}) \dot{h}^{a b}(t, \vec{x})\right\rangle
$$

for the energy density in gravitational waves (see, e.g., p. 955 of Ref. [12]). By differentiating the plane wave expansion (2.5) with respect to $t$, forming the contraction in Eq. (2.13), and calculating the expectation value using Eq. (2.12), we find

$$
\rho_{\mathrm{gw}}=\frac{4 \pi^{2} c^{2}}{G} \int_{0}^{\infty} d f f^{2} H(f) \quad\left(=: \int_{0}^{\infty} d f \frac{d \rho_{\mathrm{gw}}}{d f}\right) .
$$

Using Eqs. (2.1) and (2.2) for $\Omega_{\mathrm{gw}}(f)$ then yields

\footnotetext{
${ }^{2}$ If the stochastic background is anisotropic, we should replace $H(f)$ by a function that depends on $\hat{\Omega}$ in addition to $f$. If the stochastic background is polarized, we should replace $H(f)$ by a function that depends on the polarization $A=+, \times$ as well.
}

$$
H(f)=\frac{3 H_{0}^{2}}{32 \pi^{3}}|f|^{-3} \Omega_{\mathrm{gw}}(|f|)
$$

Thus,

$$
\begin{aligned}
& \left\langle h_{A}^{*}(f, \hat{\Omega}) h_{A^{\prime}}\left(f^{\prime}, \hat{\Omega}^{\prime}\right)\right\rangle \\
& \quad=\frac{3 H_{0}^{2}}{32 \pi^{3}} \delta^{2}\left(\hat{\Omega}, \hat{\Omega}^{\prime}\right) \delta_{A A^{\prime}} \delta\left(f-f^{\prime}\right)|f|^{-3} \Omega_{\mathrm{gw}}(|f|),
\end{aligned}
$$

which is the desired result.

\section{Observational constraints}

At present, there are three observational constraints on the stochastic gravity-wave spectrum $\Omega_{\mathrm{gw}}(f)$. These constraints are quite weak in the frequency range of interest for groundbased interferometers ( $1 \mathrm{~Hz}<f<10^{3} \mathrm{~Hz}$ ) and for proposed space-based detectors $\left(10^{-4} \mathrm{~Hz}<f<10^{-1} \mathrm{~Hz}\right)$. There are tighter constraints on the spectrum in two frequency ranges, and one "wideband"' but very weak constraint. In this paper, we simply state the constraints. For a more complete discussion, see Ref. [8] and the references mentioned therein.

(i) The strongest observational constraint on $\Omega_{\mathrm{gw}}(f)$ comes from the high degree of isotropy observed in the CMBR. In particular, the 1-yr [13,14], 2-yr [15], and 4-yr [16] data sets from the Cosmic Background Explorer (COBE) satellite place very strong restrictions on $\Omega_{\mathrm{gw}}(f)$ at very low frequencies:

$$
\Omega_{\mathrm{gw}}(f) h_{100}^{2}<7 \times 10^{-11}\left(\frac{H_{0}}{f}\right)^{2} \quad \text { for } \quad H_{0}<f<30 H_{0} .
$$

Note that the above constraint does not apply to any gravitational wave, but only to those of cosmological origin that were already present at the time of last scattering of the CMBR. Also, since $H_{0}=3.2 \times 10^{-18} h_{100} \mathrm{~Hz}$, this limit applies only over a narrow band of frequencies $\left(10^{-18} \mathrm{~Hz}\right.$ $<f<10^{-16} \mathrm{~Hz}$ ), which is far below any frequency band accessible to investigation by either Earth-based or spacebased interferometers. Thus, although this constraint is severe, it is not directly relevant for any of the present-day gravity-wave experiments.

(ii) The second observational constraint comes from almost a decade of monitoring the radio pulses arriving from a number of stable millisecond pulsars [17]. These pulsars are remarkably stable clocks, and the regularity of their pulses places tight constraints on $\Omega_{\mathrm{gw}}(f)$ at frequencies on the order of the inverse of the observation time of the pulsars $\left(\sim 10^{-8} \mathrm{~Hz}\right)$ :

$$
\Omega_{\mathrm{gw}}\left(f=10^{-8} \mathrm{~Hz}\right) h_{100}^{2}<10^{-8} .
$$

Like the constraint on the stochastic gravity-wave background from the isotropy of the CMBR, the millisecond pulsar timing constraint is irrelevant for current gravity-wave experiments. The frequency $f=10^{-8} \mathrm{~Hz}$ is 10 orders of magnitude smaller than the band of frequencies accessible to 
LIGO, VIRGO, etc., and 4 orders of magnitude smaller than that for proposed space-based detectors.

(iii) The third and final observational constraint on $\Omega_{\mathrm{gw}}(f)$ comes from the standard model of big-bang nucleosynthesis [9]. This model provides remarkably accurate fits to the observed abundances of the light elements in the universe, tightly constraining a number of key cosmological parameters. One of the parameters constrained in this way is the expansion rate of the universe at the time of nucleosynthesis. This places a constraint on the energy density of the universe at that time, which in turn constrains the energy density in a cosmological background of gravitational radiation:

$$
\int_{f>10^{-8} \mathrm{~Hz}} d \ln f \Omega_{\mathrm{gw}}(f) h_{100}^{2}<10^{-5} .
$$

Although this bound constrains the spectrum of gravitational radiation $\Omega_{\mathrm{gw}}(f)$ over a broad range of frequencies, it is not very restrictive.

\section{STOCHASTIC BACKGROUND: DETECTION}

In this section, we begin our detailed discussion of the detection of a stochastic background of gravitational radiation. We explain how one can correlate the outputs of two gravity-wave detectors to detect (or put an upper limit on) a stochastic background signal. In Sec. III B, we give a detailed derivation of the overlap reduction function that covers the case where the two arms of a detector are not perpendicular (e.g., GEO-600). In Sec. III C, we give a rigorous derivation of the optimal signal processing strategy. The statistical assumptions that we will use for the stochastic gravity-wave background are those described in Sec. II B. In addition, we will assume that the noises intrinsic to the detectors are (i) stationary, (ii) Gaussian, (iii) statistically independent of one another and of the stochastic gravity-wave background, and (iv) much larger in magnitude than the stochastic gravity-wave background. The modifications that are necessary when one relaxes most of these assumptions will be discussed in Sec. V.

\section{A. Coincident and coaligned detectors}

To begin, let us consider the simplest possible case. Let us suppose that we have two coincident and coaligned gravitywave detectors with outputs

$$
\begin{aligned}
& s_{1}(t):=h_{1}(t)+n_{1}(t), \\
& s_{2}(t):=h_{2}(t)+n_{2}(t) .
\end{aligned}
$$

Here $h_{1}(t)$ and $h_{2}(t)$ denote the gravitational strains in the two detectors due to the stochastic background, and $n_{1}(t)$ and $n_{2}(t)$ denote the noises intrinsic to the first and second detectors, respectively. ${ }^{3}$ Since we are assuming that the two

\footnotetext{
${ }^{3} \mathrm{We}$ will assume throughout that the detector outputs are not whitened.
}

detectors are coincident and coaligned (i.e., have identical locations and arm orientations), the gravitational strains are identical:

$$
h(t):=h_{1}(t)=h_{2}(t) .
$$

But the noises $n_{1}(t)$ and $n_{2}(t)$ are not equal to one another. As mentioned above, we will assume that they are stationary, Gaussian, statistically independent of one another and of the gravitational strains, and much larger in magnitude than the gravitational strains. ${ }^{4}$

Given the detector outputs $s_{1}(t)$ and $s_{2}(t)$, we can form a product "signal" $S$ by multiplying them together and integrating over time:

$$
S:=\int_{-T / 2}^{T / 2} d t s_{1}(t) s_{2}(t)
$$

This quantity is proportional to the (zero-lag) crosscorrelation of $s_{1}(t)$ and $s_{2}(t)$ for an observation time $T$. Since $s_{1}(t)$ and $s_{2}(t)$ are random variables, so too is $S$. It has a mean value

$$
\mu:=\langle S\rangle
$$

and variance

$$
\sigma^{2}:=\left\langle S^{2}\right\rangle-\langle S\rangle^{2},
$$

which are related to the variances of $n_{1}(t), n_{2}(t)$, and $h(t) .{ }^{5}$ The goal is to calculate $\mu$ and $\sigma$, and then to construct the signal-to-noise ratio

$$
\mathrm{SNR}:=\frac{\mu}{\sigma}
$$

As we shall see in Sec. IV, the value of the signal-to-noise ratio enters the decision rule for the detection of a stochastic gravity-wave signal.

\footnotetext{
${ }^{4}$ The assumption that the noises intrinsic to the detectors are statistically independent of one another is unrealistic for the case of coincident and coaligned detectors. But it is a reasonable assumption for widely separated detector sites. (See Sec. V E for more details.)

${ }^{5}$ The mean values of $n_{1}(t), n_{2}(t)$, and $h(t)$ are equal to zero, either by assumption or by definition.
} 
Let us start with the mean value $\mu$. By definition,

$$
\begin{aligned}
\mu:=\langle S\rangle= & \int_{-T / 2}^{T / 2} d t\left\langle s_{1}(t) s_{2}(t)\right\rangle \\
= & \int_{-T / 2}^{T / 2} d t\left\langle h^{2}(t)+h(t) n_{2}(t)\right. \\
& \left.+n_{1}(t) h(t)+n_{1}(t) n_{2}(t)\right\rangle \\
= & \int_{-T / 2}^{T / 2} d t\left\langle h^{2}(t)\right\rangle \\
= & T\left\langle h^{2}(t)\right\rangle=: T \sigma_{h}^{2}
\end{aligned}
$$

where $\sigma_{h}^{2}$ denotes the (time-independent) variance of $h(t) .{ }^{6}$ Note that we used the statistical independence of $n_{1}(t), n_{2}(t)$, and $h(t)$ to obtain the third line, and the stationarity of $h(t)$ to obtain the last.

To express the variance $\sigma_{h}^{2}:=\left\langle h^{2}(t)\right\rangle$ in terms of the frequency spectrum $\Omega_{\mathrm{gw}}(f)$, we will make use of the plane wave expansion (2.5) and the expectation value (2.16). Since

$$
h(t):=h_{a b}\left(t, \vec{x}_{0}\right) \frac{1}{2}\left(\hat{X}^{a} \hat{X}^{b}-\hat{Y}^{a} \hat{Y}^{b}\right)
$$

(where $\vec{x}_{0}$ is the common position vector of the central station of the two coincident and coaligned detectors, and $\hat{X}^{a}$ and $\hat{Y}^{a}$ are unit vectors pointing in the directions of the detector arms), ${ }^{7}$ it follows that

$$
\begin{aligned}
\sigma_{h}^{2}= & \sum_{A} \sum_{A^{\prime}} \int_{S^{2}} d \hat{\Omega} \int_{S^{2}} d \hat{\Omega}^{\prime} \int_{-\infty}^{\infty} d f \int_{-\infty}^{\infty} d f^{\prime} \\
& \times\left\langle h_{A}^{*}(f, \hat{\Omega}) h_{A^{\prime}}\left(f^{\prime}, \hat{\Omega}^{\prime}\right)\right\rangle e^{-i 2 \pi f\left(t-\hat{\Omega} \cdot \vec{x}_{0} / c\right)} \\
& \times e^{i 2 \pi f^{\prime}\left(t-\hat{\Omega}^{\prime} \cdot \vec{x}_{0} / c\right)} F^{A}(\hat{\Omega}) F^{A^{\prime}}\left(\hat{\Omega}^{\prime}\right),
\end{aligned}
$$

where

$$
F^{A}(\hat{\Omega}):=e_{a b}^{A}(\hat{\Omega}) \frac{1}{2}\left(\hat{X}^{a} \hat{X}^{b}-\hat{Y}^{a} \hat{Y}^{b}\right)
$$

\footnotetext{
${ }^{6}$ The dimensions of $\sigma_{h}^{2}$ and $\sigma^{2}$ are different: $\sigma_{h}^{2}$ has dimensions of strain ${ }^{2}$, while $\sigma^{2}$ has dimensions of $\operatorname{strain}^{4} \mathrm{sec}^{2}$. [See, e.g., Eq. (3.20).]

${ }^{7} \vec{x}_{0}$ and $\hat{X}^{a}, \hat{Y}^{a}$ are actually functions of time due to the Earth's motion with respect to the cosmological rest frame. They can be treated as constants, however, since (i) the velocity of the Earth with respect to the cosmological rest frame is small compared to the speed of light and (ii) the distance that the central stations and arms move during the correlation time between the two detectors is small compared to the arm length. (The correlation time equals zero for coincident and coaligned detectors; it equals the light travel time between the two detectors when the detectors are spatially separated.) See Ref. [10] for more details.
}

is the response of either detector to a zero frequency, unit amplitude, $A=+, \times$ polarized gravitational wave. Using Eq. (2.16) for the expectation value $\left\langle h_{A}^{*}(f, \hat{\Omega}) h_{A^{\prime}}\left(f^{\prime}, \hat{\Omega}^{\prime}\right)\right\rangle$, the above expression for $\sigma_{h}^{2}$ simplifies to

$$
\begin{aligned}
\sigma_{h}^{2} & =\frac{3 H_{0}^{2}}{32 \pi^{3}} \int_{-\infty}^{\infty} d f|f|^{-3} \Omega_{\mathrm{gw}}(|f|) \sum_{A} \int_{S^{2}} d \hat{\Omega} F^{A}(\hat{\Omega}) F^{A}(\hat{\Omega}) \\
& =\frac{3 H_{0}^{2}}{20 \pi^{2}} \int_{-\infty}^{\infty} d f|f|^{-3} \Omega_{\mathrm{gw}}(|f|),
\end{aligned}
$$

where we used

$$
\sum_{A} \int_{S^{2}} d \hat{\Omega} F^{A}(\hat{\Omega}) F^{A}(\hat{\Omega})=\frac{8 \pi}{5}
$$

to obtain the last line. Thus, for coincident and coaligned detectors, the mean value of the cross-correlation signal $S$ is

$$
\mu=\frac{3 H_{0}^{2}}{20 \pi^{2}} T \int_{-\infty}^{\infty} d f|f|^{-3} \Omega_{\mathrm{gw}}(|f|) .
$$

This is the first of our desired results.

To evaluate the variance $\sigma^{2}$, we will make use of the assumption that the noises intrinsic to the detectors are much larger in magnitude than the gravitational strains. Then

$$
\begin{aligned}
\sigma^{2} & :=\left\langle S^{2}\right\rangle-\langle S\rangle^{2} \approx\left\langle S^{2}\right\rangle \\
& =\int_{-T / 2}^{T / 2} d t \int_{-T / 2}^{T / 2} d t^{\prime}\left\langle s_{1}(t) s_{2}(t) s_{1}\left(t^{\prime}\right) s_{2}\left(t^{\prime}\right)\right\rangle \\
& \approx \int_{-T / 2}^{T / 2} d t \int_{-T / 2}^{T / 2} d t^{\prime}\left\langle n_{1}(t) n_{2}(t) n_{1}\left(t^{\prime}\right) n_{2}\left(t^{\prime}\right)\right\rangle \\
& =\int_{-T / 2}^{T / 2} d t \int_{-T / 2}^{T / 2} d t^{\prime}\left\langle n_{1}(t) n_{1}\left(t^{\prime}\right)\right\rangle\left\langle n_{2}(t) n_{2}\left(t^{\prime}\right)\right\rangle,
\end{aligned}
$$

where we used the statistical independence of $n_{1}(t)$ and $n_{2}(t)$ to obtain the last line. By definition, ${ }^{8}$

$$
\left\langle n_{i}(t) n_{i}\left(t^{\prime}\right)\right\rangle=: \frac{1}{2} \int_{-\infty}^{\infty} d f e^{i 2 \pi f\left(t-t^{\prime}\right)} P_{i}(|f|),
$$

where $P_{i}(|f|)$ is the (one-sided) noise power spectrum of the $i$ th detector $(i=1,2) . \quad P_{i}(|f|)$ is a real, non-negative func-

${ }^{8}$ Equation (3.23) can also be written in the frequency domain:

$$
\left\langle\tilde{n}_{i}^{*}(f) \tilde{n}_{i}\left(f^{\prime}\right)\right\rangle=\frac{1}{2} \delta\left(f-f^{\prime}\right) P_{i}(|f|) .
$$

See the discussion surrounding Eq. (3.64) for more details. 


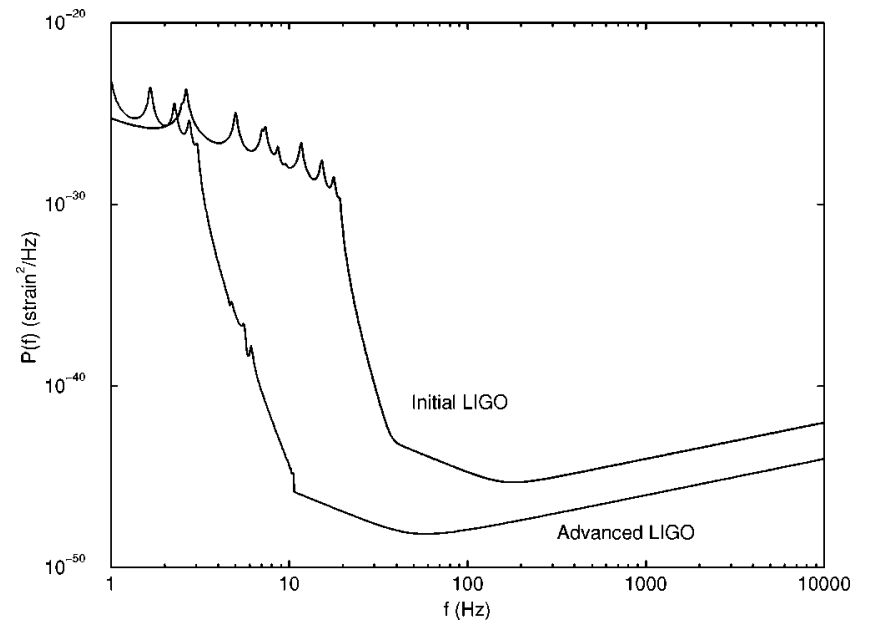

FIG. 1. A log-log plot of the predicted noise power spectra for the initial and advanced LIGO detectors. The data for these noise power spectra were taken from the published design goals [1].

tion, defined with a factor of $1 / 2$ to agree with the standard (one-sided) definition used by instrument builders. It satisfies $^{9}$

$$
\sigma_{n_{i}}^{2}:=\left\langle n_{i}^{2}(t)\right\rangle=\int_{0}^{\infty} d f P_{i}(f),
$$

and so the total noise power is the integral of $P_{i}(f)$ over all positive frequencies $f$ from 0 to $\infty$, not from $-\infty$ to $\infty$. (Hence the reason for the name one-sided.) Graphs of the predicted noise power spectra for the initial and advanced LIGO detectors are shown in Fig. 1. Graphs of the predicted noise power spectra for the other major interferometers (i.e., VIRGO, GEO-600, and TAMA-300) and for the "enhanced" LIGO detectors are shown in Figs. 11-15 in Sec. VI A.

Inserting Eq. (3.23) into Eq. (3.22) yields

$$
\begin{aligned}
\sigma^{2} \approx & \frac{1}{4} \int_{-T / 2}^{T / 2} d t \int_{-T / 2}^{T / 2} d t^{\prime} \int_{-\infty}^{\infty} d f \int_{-\infty}^{\infty} d f^{\prime} e^{i 2 \pi f\left(t-t^{\prime}\right)} \\
& \times e^{-i 2 \pi f^{\prime}\left(t-t^{\prime}\right)} P_{1}(|f|) P_{2}\left(\left|f^{\prime}\right|\right)
\end{aligned}
$$

where we used the reality of $n_{2}(t)$ and $P_{2}(|f|)$ to produce the minus sign in the power of the second exponential. If we integrate this expression over $t$ and $t^{\prime}$, we find

$$
\sigma^{2} \approx \frac{1}{4} \int_{-\infty}^{\infty} d f \int_{-\infty}^{\infty} d f^{\prime} \delta_{T}^{2}\left(f-f^{\prime}\right) P_{1}(|f|) P_{2}\left(\left|f^{\prime}\right|\right),
$$

where

$$
\delta_{T}(f):=\int_{-T / 2}^{T / 2} d t e^{-i 2 \pi f t}=\frac{\sin (\pi f T)}{\pi f}
$$

${ }^{9}$ Unlike $\sigma_{h}^{2}$ and $\sigma^{2}, \sigma_{h}^{2}$ and $\sigma_{n_{i}}^{2}$ have the same dimensions
$\left(\operatorname{strain}^{2}\right)$. is a finite-time approximation to the Dirac delta function $\delta(f)$. In the limit $T \rightarrow \infty, \delta_{T}(f)$ reduces to $\delta(f)$, but for a finite observation time $T$, one has $\delta_{T}(0)=T$. Since in practice the observation time $T$ will be large enough so that $\delta_{T}\left(f-f^{\prime}\right)$ is sharply peaked over a region in $f-f^{\prime}$ whose size $\approx 1 / T$ is very small compared to the scale on which the functions $P_{1}(|f|)$ and $P_{2}(|f|)$ are varying, ${ }^{10}$ we can replace one of the finite-time delta functions $\delta_{T}\left(f-f^{\prime}\right)$ by an ordinary Dirac delta function, and evaluate the other at $f=f^{\prime}$. Doing this yields

$$
\sigma^{2} \approx \frac{T}{4} \int_{-\infty}^{\infty} d f P_{1}(|f|) P_{2}(|f|),
$$

which is the second of our desired results.

Using Eq. (3.18) and (3.28), we can now form the signalto-noise ratio ${ }^{11}$

$$
\mathrm{SNR}:=\frac{\mu}{\sigma} \approx \frac{3 H_{0}^{2}}{10 \pi^{2}} \sqrt{T} \frac{\int_{-\infty}^{\infty} d f|f|^{-3} \Omega_{\mathrm{gw}}(|f|)}{\left[\int_{-\infty}^{\infty} d f P_{1}(|f|) P_{2}(|f|)\right]^{1 / 2}} .
$$

The multiplicative factor of $\sqrt{T}$ means that we can always exceed any prescribed value of the signal-to-noise ratio by correlating the outputs of two gravity-wave detectors for a long enough period of time. ${ }^{12} \mathrm{We}$ will have more to say about signal detection, parameter estimation, and sensitivity levels for stochastic background searches in Sec. IV.

\section{B. Overlap reduction function}

To provide a rigorous treatment of the signal analysis for a stochastic background of gravitational radiation, we must take into account the fact that the two gravity-wave detectors will not necessarily be either coincident or coaligned. There will be a reduction in sensitivity due to (i) the separation time delay between the two detectors and (ii) the non-parallel alignment of the detector arms. These two effects imply that $h_{1}(t)$ and $h_{2}(t)$ are no longer equal; the overlap between the gravitational strains in the two detectors is only partial. Statistically, these effects are most apparent in the frequency domain.

\footnotetext{
${ }^{10}$ Typically, an observation time $T$ will be on the order of months (i.e., $10^{7} \mathrm{sec}$ ), while the noise power spectra $P_{i}(|f|)$ vary on a scale of greater than a few $\mathrm{Hz}$.

${ }^{11} \mathrm{Be}$ warned that the signal processing strategy described above, leading to Eq. (3.29), is not optimal even for the case of coincident and coaligned detectors. The optimal signal processing strategy, which is described in Sec. III C, leads to the signal-to-noise ratio given by Eq. (3.75). Setting $\gamma(f)=1$ in Eq. (3.75) yields an expression for the optimally filtered signal-to-noise ratio for the case of coincident and coaligned detectors.

${ }^{12}$ This assumes that there is no systematic source of correlated detector noise. In Sec. V E, we discuss the limits that correlated detector noise imposes.
} 

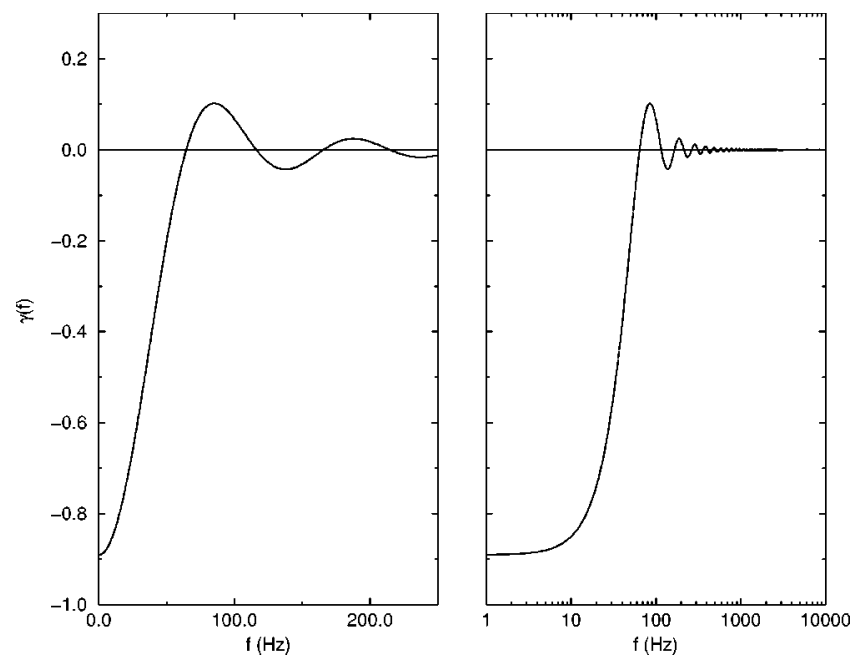

FIG. 2. The overlap reduction function $\gamma(f)$ for the Hanford, WA and Livingston, LA LIGO detector pair. (The horizontal axis of the left-hand graph is linear, while that of the right-hand graph is $\log _{10}$.) The overlap reduction function has its first zero at $64 \mathrm{~Hz}$, as explained in the text. It falls off rapidly at higher frequencies.

The overlap reduction function $\gamma(f)$, first calculated in closed form by Flanagan [7], quantifies these two effects. This is a dimensionless function of frequency $f$, which is determined by the relative positions and orientations of a pair of detectors. Explicitly,

$$
\gamma(f):=\frac{5}{8 \pi} \sum_{A} \int_{S^{2}} d \hat{\Omega} e^{i 2 \pi f \hat{\Omega} \cdot \Delta \vec{x} / c} F_{1}^{A}(\hat{\Omega}) F_{2}^{A}(\hat{\Omega}),
$$

where $\hat{\Omega}$ is a unit vector specifying a direction on the twosphere, $\Delta \vec{x}:=\vec{x}_{1}-\vec{x}_{2}$ is the separation vector between the central stations of the two detector sites, and

$$
F_{i}^{A}(\hat{\Omega}):=e_{a b}^{A}(\hat{\Omega}) d_{i}^{a b}:=e_{a b}^{A}(\hat{\Omega}) \frac{1}{2}\left(\hat{X}_{i}^{a} \hat{X}_{i}^{b}-\hat{Y}_{i}^{a} \hat{Y}_{i}^{b}\right)
$$

is the response of the $i$ th detector $(i=1,2)$ to the $A=+, \times$ polarization. [See also Eq. (3.14).] The symmetric, trace-free tensor $d_{i}^{a b}$ specifies the orientation of the two arms of the $i$ th detector. The overlap reduction function $\gamma(f)$ equals unity for coincident and coaligned detectors. It decreases below unity when the detectors are shifted apart (so there is a phase shift between the signals in the two detectors) or rotated out of coalignment (so the detectors are sensitive to different polarizations). In Sec. III C, we will see that $\gamma(f)$ arises naturally when evaluating the expectation value of the product of the gravitational strains at two different detectors when they are driven by an isotropic and unpolarized stochastic background of gravitational radiation.

To get a better feeling for the meaning of $\gamma(f)$, let us look at each term in Eq. (3.30) separately: (i) The overall normalization factor $5 / 8 \pi$ is chosen so that for a pair of coincident and coaligned detectors $\gamma(f)=1$ for all frequencies $f$. (ii) The sum over polarizations $A$ is appropriate for an unpolarized stochastic background. (iii) The integral over the

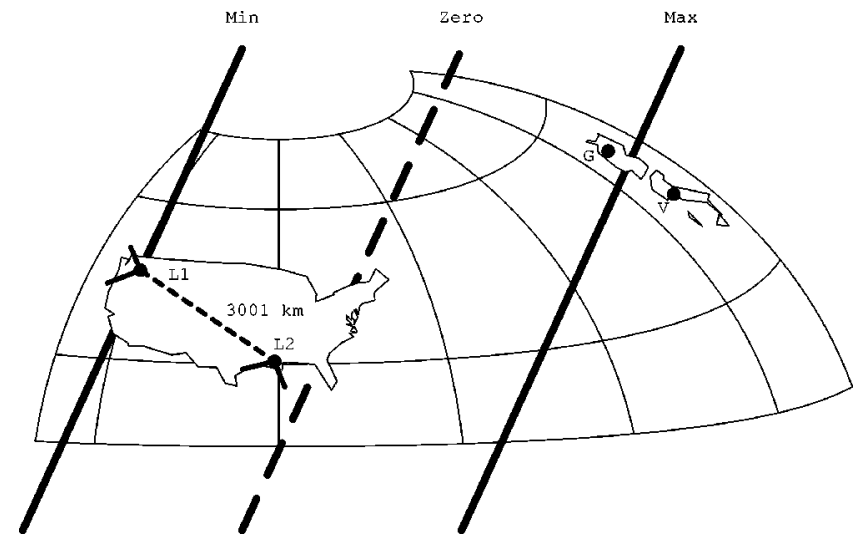

FIG. 3. The surface of the Earth $\left(15^{\circ}<\right.$ latitude $<75^{\circ},-130^{\circ}$ $<$ longitude $<20^{\circ}$ ) including the LIGO detectors in Hanford, WA (L1) and Livingston, LA (L2), the VIRGO detector (V) in Pisa, Italy, and the GEO-600 (G) detector in Hanover, Germany. The perpendicular arms of the LIGO detectors are also illustrated (though not to scale). A plane gravitational wave passing by the Earth is indicated by successive minimum, zero, and maximum of the wave. As this wave passes by the pair of LIGO detectors, it excites the two in coincidence at the moment shown, since both detectors are driven negative by the wave. During the time when the zero is between L1 and L2, the two detectors respond in anticoincidence. Provided that the wavelength of the incident gravitational wave is larger than twice the separation $(d=3001 \mathrm{~km})$ between the detectors, the two detectors are driven in coincidence more of the time than in anti-coincidence.

two-sphere is an isotropic average over all directions $\hat{\Omega}$ of the incoming radiation. (iv) The exponential phase factor is the phase shift arising from the time delay between the two detectors for radiation arriving along the direction $\hat{\Omega}$. In the limit $f \rightarrow 0$, this phase shift also goes to zero, and the two detectors become effectively coincident. (v) The quantity $\Sigma_{A} F_{1}^{A}(\hat{\Omega}) F_{2}^{A}(\hat{\Omega})$ is the sum of products of the responses of the two detectors to the + and $\times$ polarization waves. For coaligned detectors, $F_{1}^{A}(\hat{\Omega})=F_{2}^{A}(\hat{\Omega})$ and the integral of this quantity over the two-sphere equals the inverse of the overall normalization factor. [See Eq. (3.17).]

Figure 2 shows a graph of the overlap reduction function $\gamma(f)$ for the Hanford, WA and Livingston, LA LIGO detector pair. ${ }^{13}$ Note that the overlap reduction function for the LIGO detector pair is negative as $f \rightarrow 0$. This is because the arm orientations of the two LIGO detectors are not parallel to one another, but are rotated by $90^{\circ}$. If, for example, the Livingston, LA detector arms were rotated by $90^{\circ}$ in the clockwise direction, only the overall sign of $\gamma(f)$ would change. Note also that the magnitude of $\gamma(0)$ is not unity, because the planes of the Hanford, WA and Livingston, LA detectors are not identical. ${ }^{14}$ Thus, the arms of the two detectors are not exactly parallel, and $|\gamma(0)|=0.89$, which is less than 1.

\footnotetext{
${ }^{13}$ Figures $16-20$ in Sec. VI B show graphs of the overlap reduction functions for different detector pairs.

${ }^{14}$ The two LIGO detectors are separated by an angle of $27.2^{\circ}$ as seen from the center of the Earth.
} 
From Fig. 2, one also sees that the overlap reduction function for the two LIGO detectors has its first zero at $64 \mathrm{~Hz}$. This can be explained by the fact that a gravitational plane wave passing by the Earth excites a pair of detectors in coincidence when the positive (or negative) amplitude part of the wave is passing by both detectors at the same time; it excites the two detectors in anti-coincidence when the positive (or negative) amplitude part of the wave is passing by one detector, and the negative (or positive) amplitude part of the wave is passing by the other detector. (See Fig. 3.) Provided that the wavelength of the incident gravitational wave is larger than twice the distance between the two detectors, the detectors will be driven in coincidence (on average). For the case of the LIGO detector pair, this means that the Hanford, WA and Livingston, LA detectors will be driven in coincidence (on the average) by an isotropic and unpolarized stochastic background of gravitational radiation having a frequency of less than $f=c /(2 d)=50 \mathrm{~Hz}$. The actual frequency of the zero $(f=64 \mathrm{~Hz})$ is slightly larger than this, since $\gamma(f)$ is a sum of three spherical Bessel functions, which does not vanish at exactly $50 \mathrm{~Hz}$.

In Appendix B of Ref. [7], Flanagan outlines a derivation of a closed-form expression for the overlap reduction function $\gamma(f)$. The resulting expression applies to any pair of gravity-wave detectors, including interferometers with nonperpendicular arms and/or arbitrary orientations. This is a useful result, because, e.g., the arms of the GEO-600 detector are separated by $94.33^{\circ}$. Below we give a more detailed version of the derivation that appears in Ref. [7], and correct a typographical error that appears in Eq. (B6) of that paper.

We take, as our starting point for the derivation, the integral expression (3.30) for $\gamma(f)$. To simplify the notation in what follows, we also define

$$
\Delta \vec{x}:=d \hat{s} \text { and } \alpha:=\frac{2 \pi f d}{c},
$$

where $\hat{s}$ is a unit vector that points in the direction connecting the two detectors, and $d$ is the distance between the two detectors. In terms of these quantities, we can write

$$
\gamma(f)=d_{1}^{a b} d_{2}^{c d} \Gamma_{a b c d}(\alpha, \hat{s}),
$$

where

$$
\Gamma_{a b c d}(\alpha, \hat{s}):=\frac{5}{8 \pi} \sum_{A} \int_{S^{2}} d \hat{\Omega} e^{i \alpha \hat{\Omega} \cdot \hat{s}} e_{a b}^{A}(\hat{\Omega}) e_{c d}^{A}(\hat{\Omega}) .
$$

$\Gamma_{a b c d}(\alpha, \hat{s})$ is a tensor which is symmetric under the interchanges $a \leftrightarrow b, c \leftrightarrow d, a b \leftrightarrow c d$. It is also trace-free with respect to the $a b$ and $c d$ index pairs.

To evaluate $\Gamma_{a b c d}(\alpha, \hat{s})$, we begin by writing down the most general tensor constructed from $\delta_{a b}$ and $s_{a}$ that has the above-mentioned symmetry properties:

$$
\begin{aligned}
\Gamma_{a b c d}(\alpha, \hat{s})= & A(\alpha) \delta_{a b} \delta_{c d}+B(\alpha)\left(\delta_{a c} \delta_{b d}+\delta_{b c} \delta_{a d}\right) \\
& +C(\alpha)\left(\delta_{a b} s_{c} s_{d}+\delta_{c d} s_{a} s_{b}\right) \\
& +D(\alpha)\left(\delta_{a c} s_{b} s_{d}+\delta_{a d} s_{b} s_{c}\right. \\
& \left.+\delta_{b c} s_{a} s_{d}+\delta_{b d} s_{a} s_{c}\right)+E(\alpha) s_{a} s_{b} s_{c} s_{d}
\end{aligned}
$$

We then contract Eq. (3.35) with $\delta^{a b} \delta^{c d},\left(\delta^{a c} \delta^{b d}\right.$ $\left.+\delta^{b c} \delta^{a d}\right), \ldots, s^{a} s^{b} s^{c} s^{d}$ to obtain a linear system of equations for the functions $A, B, \ldots, E$ :

$$
\left[\begin{array}{rrrrr}
9 & 6 & 6 & 4 & 1 \\
6 & 24 & 4 & 16 & 2 \\
6 & 4 & 8 & 8 & 2 \\
4 & 16 & 8 & 24 & 4 \\
1 & 2 & 2 & 4 & 1
\end{array}\right]\left[\begin{array}{c}
A \\
B \\
C \\
D \\
E
\end{array}\right](\alpha)=\left[\begin{array}{c}
p \\
q \\
r \\
s \\
t
\end{array}\right](\alpha)
$$

where

$$
\begin{aligned}
p(\alpha):= & \Gamma_{a b c d}(\alpha, \hat{s}) \delta^{a b} \delta^{c d}, \\
q(\alpha):= & \Gamma_{a b c d}(\alpha, \hat{s})\left(\delta^{a c} \delta^{b d}+\delta^{b c} \delta^{a d}\right), \\
r(\alpha):= & \Gamma_{a b c d}(\alpha, \hat{s})\left(\delta^{a b} s^{c} s^{d}+\delta^{c d} s^{a} s^{b}\right), \\
s(\alpha):= & \Gamma_{a b c d}(\alpha, \hat{s})\left(\delta^{a c} s^{b} s^{d}+\delta^{a d} s^{b} s^{c}+\delta^{b c} s^{a} s^{d}\right. \\
& \left.+\delta^{b d} s^{a} s^{c}\right), \\
t(\alpha):= & \Gamma_{a b c d}(\alpha, \hat{s}) s^{a} s^{b} s^{c} s^{d} .
\end{aligned}
$$

From Eq. (3.34), we see that the functions $p, q, \ldots, t$ are scalar integrals that involve contractions of the spin-two polarization tensors $e_{a b}^{A}(\hat{\Omega})$.

To evaluate these integrals, we choose (without loss of generality) a coordinate system where the unit vector $\hat{s}$ coincides with unit vector $\hat{z}$. Then

$$
\hat{\Omega} \cdot \hat{s}=\cos \theta, \quad \hat{m} \cdot \hat{s}=0, \quad \hat{n} \cdot \hat{s}=-\sin \theta,
$$

and

$$
\begin{aligned}
& p(\alpha)=0, \\
& q(\alpha)=10 \int_{-1}^{1} d x e^{i \alpha x}=20 j_{0}(\alpha), \\
& r(\alpha)=0, \\
& s(\alpha)=10 \int_{-1}^{1} d x e^{i \alpha x}\left(1-x^{2}\right)=\frac{40}{\alpha} j_{1}(\alpha), \\
& t(\alpha)=\frac{5}{4} \int_{-1}^{1} d x e^{i \alpha x}\left(1-x^{2}\right)^{2}=\frac{20}{\alpha^{2}} j_{2}(\alpha),
\end{aligned}
$$


where $j_{0}(\alpha), j_{1}(\alpha)$, and $j_{2}(\alpha)$ are the standard spherical Bessel functions:

$$
\begin{aligned}
& j_{0}(\alpha)=\frac{\sin \alpha}{\alpha}, \\
& j_{1}(\alpha)=\frac{\sin \alpha}{\alpha^{2}}-\frac{\cos \alpha}{\alpha}, \\
& j_{2}(\alpha)=3 \frac{\sin \alpha}{\alpha^{3}}-3 \frac{\cos \alpha}{\alpha^{2}}-\frac{\sin \alpha}{\alpha} .
\end{aligned}
$$

Note that $p(\alpha)=0$ and $r(\alpha)=0$ are immediate consequences of the trace-free property of $\Gamma_{a b c d}(\alpha, \hat{s})$.

The above linear system of equations (3.36) can be inverted for the functions $A, B, \ldots, E$. The results are

$$
\left[\begin{array}{c}
A \\
B \\
C \\
D \\
E
\end{array}\right](\alpha)=\frac{1}{2 \alpha^{2}}\left[\begin{array}{rrr}
-5 \alpha^{2} & 10 \alpha & 5 \\
5 \alpha^{2} & -10 \alpha & 5 \\
5 \alpha^{2} & -10 \alpha & -25 \\
-5 \alpha^{2} & 20 \alpha & -25 \\
5 \alpha^{2} & -50 \alpha & 175
\end{array}\right]\left[\begin{array}{c}
j_{0} \\
j_{1} \\
j_{2}
\end{array}\right](\alpha)
$$

Finally, to obtain an expression for the overlap reduction function $\gamma(f)$, we substitute Eq. (3.35) into Eq. (3.33). Since $d_{i}^{a b}(i=1,2)$ is trace-free, it follows that

$$
\begin{aligned}
\gamma(f)= & 2 B(\alpha) d_{1}^{a b} d_{2 a b}+4 D(\alpha) d_{1}^{a b} d_{2 a}{ }^{c} s_{b} s_{c} \\
& +E(\alpha) d_{1}^{a b} d_{2}^{c d} s_{a} s_{b} s_{c} s_{d} .
\end{aligned}
$$

Substituting the expressions for the functions $B, D, E$ given by Eq. (3.41) into Eq. (3.42) yields

$$
\begin{aligned}
\gamma(f)= & \rho_{1}(\alpha) d_{1}^{a b} d_{2 a b}+\rho_{2}(\alpha) d_{1}^{a b} d_{2 a}{ }^{c} s_{b} s_{c} \\
& +\rho_{3}(\alpha) d_{1}^{a b} d_{2}^{c d} s_{a} s_{b} s_{c} s_{d},
\end{aligned}
$$

where

$$
\left[\begin{array}{l}
\rho_{1} \\
\rho_{2} \\
\rho_{3}
\end{array}\right](\alpha)=\frac{1}{2 \alpha^{2}}\left[\begin{array}{rrr}
10 \alpha^{2} & -20 \alpha & 10 \\
-20 \alpha^{2} & 80 \alpha & -100 \\
5 \alpha^{2} & -50 \alpha & 175
\end{array}\right]\left[\begin{array}{c}
j_{0} \\
j_{1} \\
j_{2}
\end{array}\right](\alpha)
$$

This is the desired result.

Note that in Eq. (B6) of Ref. [7], the factor multiplying $j_{1}(\alpha)$ in $\rho_{1}(\alpha)$ is $-2 / \alpha$. As shown in Eq. (3.44), this factor should equal $-10 / \alpha$.

\section{Optimal filtering}

Using the techniques developed in the previous two subsections, we are now in a position to give a rigorous derivation of the optimal signal processing required for the detection of a stochastic background of gravitational radiation. We start by writing the cross-correlation signal $S$ between the outputs of the two detectors in the following form:

$$
S:=\int_{-T / 2}^{T / 2} d t \int_{-T / 2}^{T / 2} d t^{\prime} s_{1}(t) s_{2}\left(t^{\prime}\right) Q\left(t, t^{\prime}\right)
$$

where as before

$$
\begin{aligned}
& s_{1}(t):=h_{1}(t)+n_{1}(t), \\
& s_{2}(t):=h_{2}(t)+n_{2}(t),
\end{aligned}
$$

but now $Q\left(t, t^{\prime}\right)$ is a filter function, which is not necessarily equal to $\delta\left(t-t^{\prime}\right)$ as we assumed in Sec. III A. Because we are assuming in this section that the statistical properties of the stochastic gravity-wave background and noise intrinsic to the detectors are both stationary, the best choice of filter function $Q\left(t, t^{\prime}\right)$ can depend only upon the time difference $\Delta t:=t-t^{\prime}$. The goal is to find the optimal choice of filter function $Q\left(t-t^{\prime}\right):=Q\left(t, t^{\prime}\right)$ in a rigorous way.

The optimal choice of filter function $Q\left(t-t^{\prime}\right)$ will depend upon the locations and orientations of the detectors, as well as on the spectrum of the stochastic gravity-wave background and the noise power spectra of the detectors. It falls off rapidly to zero for time delays $\Delta t=t-t^{\prime}$ whose magnitude is large compared to the light travel time $d / c$ between the two sites. ${ }^{15}$ (See Fig. 5, which is located at the end of this section.) Since a typical observation time $T$ will be $\gg d / c$, we are justified in changing the limits on one of the integrations in Eq. (3.45) to obtain

$$
S=\int_{-T / 2}^{T / 2} d t \int_{-\infty}^{\infty} d t^{\prime} s_{1}(t) s_{2}\left(t^{\prime}\right) Q\left(t-t^{\prime}\right)
$$

This change of limits simplifies the mathematical analysis that follows.

We can also write Eq. (3.48) in the frequency domain. Using the convention

$$
\tilde{g}(f):=\int_{-\infty}^{\infty} d t e^{-i 2 \pi f t} g(t)
$$

for the Fourier transform of $g(t)$, it follows that

$$
S=\int_{-\infty}^{\infty} d f \int_{-\infty}^{\infty} d f^{\prime} \delta_{T}\left(f-f^{\prime}\right) \tilde{s}_{1}^{*}(f) \tilde{s}_{2}\left(f^{\prime}\right) \widetilde{Q}\left(f^{\prime}\right),
$$

where $\widetilde{s}_{1}(f), \widetilde{s}_{2}(f)$, and $\widetilde{Q}(f)$ are the Fourier transforms of $s_{1}(t), s_{2}(t)$, and $Q\left(t-t^{\prime}\right)$, and $\delta_{T}\left(f-f^{\prime}\right)$ is the finite-time approximation to the Dirac delta function $\delta\left(f-f^{\prime}\right)$ defined by Eq. (3.27). Note also that for a real $Q\left(t-t^{\prime}\right), \widetilde{Q}(-f)$ $=\widetilde{Q}^{*}(f)$.

${ }^{15} d / c=10^{-2}$ sec for the LIGO detector pair. 
The optimal choice of filter function also depends on the quantity that we want to maximize. As we shall see in Sec. IV, in the context of stochastic background searches, it is natural to maximize the signal-to-noise ratio

$$
\mathrm{SNR}:=\frac{\mu}{\sigma},
$$

where $\mu$ and $\sigma^{2}$ are the mean value and variance of the cross-correlation signal $S$ defined by Eqs. (3.5) and (3.6). The techniques that we will use to evaluate $\mu$ and $\sigma^{2}$ are very similar to those that we used in Sec. III A for the case of coincident and coaligned detectors.

The calculation of the mean value $\mu$ is straightforward. Since we are assuming that the noises intrinsic to the two detectors are statistically independent of each other and of the gravitational strains, it follows immediately from Eq. (3.50) that

$$
\mu:=\langle S\rangle=\int_{-\infty}^{\infty} d f \int_{-\infty}^{\infty} d f^{\prime} \delta_{T}\left(f-f^{\prime}\right)\left\langle\widetilde{h}_{1}^{*}(f) \widetilde{h}_{2}\left(f^{\prime}\right)\right\rangle \widetilde{Q}\left(f^{\prime}\right)
$$

To calculate the expectation value $\left\langle\widetilde{h}_{1}^{*}(f) \widetilde{h}_{2}\left(f^{\prime}\right)\right\rangle$, we again make use of the plane wave expansion $(2.5)$ and the expectation value (2.16). Since

$$
\widetilde{h}_{i}(f)=\sum_{A} \int_{S^{2}} d \hat{\Omega} h_{A}(f, \hat{\Omega}) e^{-i 2 \pi f \hat{\Omega} \cdot \vec{x}_{i} / c} F_{i}^{A}(\hat{\Omega})
$$

where $i=1,2$ labels the two detectors, we find

$$
\begin{aligned}
\left\langle\widetilde{h}_{1}^{*}(f) \widetilde{h}_{2}\left(f^{\prime}\right)\right\rangle & =\sum_{A} \sum_{A^{\prime}} \int_{S^{2}} d \hat{\Omega} \int_{S^{2}} d \hat{\Omega}^{\prime}\left\langle h_{A}^{*}(f, \hat{\Omega}) h_{A^{\prime}}\left(f^{\prime}, \hat{\Omega}^{\prime}\right)\right\rangle e^{i 2 \pi f \hat{\Omega} \cdot \vec{x}_{1} / c} e^{-i 2 \pi f^{\prime} \hat{\Omega}^{\prime} \cdot \vec{x}_{2} / c} F_{1}^{A}(\hat{\Omega}) F_{2}^{A^{\prime}}\left(\hat{\Omega}^{\prime}\right) \\
& =\frac{3 H_{0}^{2}}{32 \pi^{3}} \delta\left(f-f^{\prime}\right)|f|^{-3} \Omega_{\mathrm{gw}}(|f|) \sum_{A} \int_{S^{2}} d \hat{\Omega} e^{i 2 \pi f \hat{\Omega} \cdot \Delta \vec{x} / c} F_{1}^{A}(\hat{\Omega}) F_{2}^{A}(\hat{\Omega}) \\
& =\frac{3 H_{0}^{2}}{20 \pi^{2}} \delta\left(f-f^{\prime}\right)|f|^{-3} \Omega_{\mathrm{gw}}(|f|) \gamma(|f|),
\end{aligned}
$$

where we used Eq. (2.16) to obtain the second equality and the definition (3.30) of the overlap reduction function to obtain the third. Substituting Eq. (3.56) into Eq. (3.52) yields

$$
\mu=\frac{3 H_{0}^{2}}{20 \pi^{2}} T \int_{-\infty}^{\infty} d f|f|^{-3} \Omega_{\mathrm{gw}}(|f|) \gamma(|f|) \widetilde{Q}(f)
$$

The factor of $T$ on the right hand side (RHS) arises from evaluating $\delta_{T}(0)$.

Before calculating the variance $\sigma^{2}$, it is worthwhile to make a slight digression and study in more detail the expectation value (3.56) derived above. It turns out that this equation has an important physical implication. In terms of the time domain variables $h_{1}(t)$ and $h_{2}\left(t^{\prime}\right)$, Eq. (3.56) can be rewritten as

$$
\left\langle h_{1}(t) h_{2}\left(t^{\prime}\right)\right\rangle=\int_{-\infty}^{\infty} d f e^{i 2 \pi f\left(t-t^{\prime}\right)} H_{12}(f),
$$

where

$$
H_{12}(f)=\frac{3 H_{0}^{2}}{20 \pi^{2}}|f|^{-3} \Omega_{\mathrm{gw}}(|f|) \gamma(|f|) .
$$

In other words, $H_{12}(f)$ is just the Fourier transform of the cross-correlation of the gravitational strains $h_{1}(t)$ and $h_{2}\left(t^{\prime}\right)$ at the two detector sites. Moreover, since the noises intrinsic to the two detectors are statistically independent of one another and of the gravitational strains, it follows that

$$
\left\langle h_{1}(t) h_{2}\left(t^{\prime}\right)\right\rangle=\left\langle s_{1}(t) s_{2}\left(t^{\prime}\right)\right\rangle .
$$

Thus, $H_{12}(f)$ is the Fourier transform of the cross-correlation of the outputs of the two detectors. But this correlation is something that we can measure (or at least estimate) given enough data. ${ }^{16}$ This in turn implies that we can measure (or at least estimate) $\Omega_{\mathrm{gw}}(|f|)$. Explicitly, given the measured

\footnotetext{
${ }^{16}$ For example, suppose we measure the outputs of the two detectors for a total observation time of $1 \mathrm{yr}$. To estimate the crosscorrelation $\left\langle s_{1}(t) s_{2}\left(t^{\prime}\right)\right\rangle$, we simply form the products of $s_{1}(t)$ and $s_{2}\left(t^{\prime}\right)$ for all $t$ and $t^{\prime}$ having the same $\Delta t=t-t^{\prime}$ (e.g., $1 \mathrm{msec}$ ), and then average the results. We then repeat this procedure for $\Delta t$ $=2 \mathrm{msec}, 3 \mathrm{msec}$, etc.
} 
values of $\left\langle s_{1}(t) s_{2}\left(t^{\prime}\right)\right\rangle$, we take their Fourier transform (with respect $t-t^{\prime}$ ), multiply by $|f|^{3}$, and divide by $\left(3 H_{0}^{2} / 20 \pi^{2}\right) \gamma(|f|)$, to determine $\Omega_{\mathrm{gw}}(|f|)$. This will yield a good approximation to the real stochastic gravity-wave spectrum provided that the noise intrinsic to the detectors is not too large or, equivalently, if we measure the detector outputs for a long enough period of time $T$. Also, the approximation of $\Omega_{\mathrm{gw}}(|f|)$ will be best for frequencies $1 / T<f<c / d$ (where $d / c$ is the light travel time between the two detectors): For $f<1 / T$, the Fourier transform lacks sufficient time domain data to provide useful information; for $f>c / d$, the overlap reduction function quickly approaches zero, and so the division by $\gamma(f)$ makes $\Omega_{\mathrm{gw}}(|f|)$ ill-behaved.

Let us return now to the calculation of the signal-to-noise ratio $\mathrm{SNR}:=\mu / \sigma$. To calculate the variance $\sigma^{2}$, we assume (as in Sec. III A) that the noises intrinsic to the two detectors are much larger in magnitude than the stochastic gravitywave background. Then

$$
\begin{aligned}
\sigma^{2} & :=\left\langle S^{2}\right\rangle-\langle S\rangle^{2} \approx\left\langle S^{2}\right\rangle \\
& \approx \int_{-\infty}^{\infty} d f \int_{-\infty}^{\infty} d f^{\prime} \int_{-\infty}^{\infty} d k \int_{-\infty}^{\infty} d k^{\prime} \delta_{T}\left(f-f^{\prime}\right) \delta_{T}\left(k-k^{\prime}\right)\left\langle\tilde{n}_{1}^{*}(f) \tilde{n}_{2}\left(f^{\prime}\right) \tilde{n}_{1}^{*}(k) \tilde{n}_{2}\left(k^{\prime}\right)\right\rangle \widetilde{Q}\left(f^{\prime}\right) \widetilde{Q}\left(k^{\prime}\right) \\
& \approx \int_{-\infty}^{\infty} d f \int_{-\infty}^{\infty} d f^{\prime} \int_{-\infty}^{\infty} d k \int_{-\infty}^{\infty} d k^{\prime} \delta_{T}\left(f-f^{\prime}\right) \delta_{T}\left(k-k^{\prime}\right)\left\langle\tilde{n}_{1}^{*}(f) \tilde{n}_{1}(-k)\right\rangle\left\langle\tilde{n}_{2}^{*}\left(-f^{\prime}\right) \tilde{n}_{2}\left(k^{\prime}\right)\right\rangle \widetilde{Q}\left(f^{\prime}\right) \widetilde{Q}\left(k^{\prime}\right),
\end{aligned}
$$

where we used the statistical independence and reality of $n_{1}(t)$ and $n_{2}(t)$ to obtain the last line.

In Sec. III A, we defined the noise power spectrum $P_{i}(|f|)$ in terms of the expectation value $\left\langle n_{i}(t) n_{i}\left(t^{\prime}\right)\right\rangle$ of the time domain random variables $n_{i}(t)$. [See Eq. (3.23).] An analogous expression holds in the frequency domain as well. Using definition (3.49) for the Fourier transform $\tilde{n}_{i}(f)$ and definition (3.23) for the noise power spectrum $P_{i}(|f|)$, it follows that

$$
\left\langle\tilde{n}_{i}^{*}(f) \tilde{n}_{i}\left(f^{\prime}\right)\right\rangle=\frac{1}{2} \delta\left(f-f^{\prime}\right) P_{i}(|f|) .
$$

Substituting this result into Eq. (3.63) then yields

$$
\begin{aligned}
\sigma^{2} \approx & \frac{1}{4} \int_{-\infty}^{\infty} d f \int_{-\infty}^{\infty} d f^{\prime} \delta_{T}^{2}\left(f-f^{\prime}\right) \\
& \times P_{1}(|f|) P_{2}\left(\left|f^{\prime}\right|\right) \widetilde{Q}(f) \widetilde{Q}^{*}\left(f^{\prime}\right) \\
\approx & \frac{T}{4} \int_{-\infty}^{\infty} d f P_{1}(|f|) P_{2}(|f|)|\widetilde{Q}(f)|^{2},
\end{aligned}
$$

where we replaced one of the finite-time delta functions $\delta_{T}\left(f-f^{\prime}\right)$ by an ordinary Dirac delta function, and evaluated the other at $f=f^{\prime}$ to obtain the last line.

To summarize,

$$
\begin{aligned}
\mu & =\frac{3 H_{0}^{2}}{20 \pi^{2}} T \int_{-\infty}^{\infty} d f|f|^{-3} \Omega_{\mathrm{gw}}(|f|) \gamma(|f|) \widetilde{Q}(f), \\
\sigma^{2} & \approx \frac{T}{4} \int_{-\infty}^{\infty} d f P_{1}(|f|) P_{2}(|f|)|\widetilde{Q}(f)|^{2} .
\end{aligned}
$$

The problem now is to find the filter function $\widetilde{Q}(f)$ that maximizes the signal-to-noise ratio (3.51), with $\mu$ and $\sigma^{2}$ as given above. This turns out to be remarkably simple if we first introduce an inner product $(A, B)$ for any pair of complex functions $A(f)$ and $B(f)$. The inner product of $A(f)$ and $B(f)$ is a complex number defined by

$$
(A, B):=\int_{-\infty}^{\infty} d f A^{*}(f) B(f) P_{1}(|f|) P_{2}(|f|) .
$$

Since $P_{i}(|f|)>0$, it follows that $(A, A) \geqslant 0$, and $(A, A)=0$ if and only if $A(f)=0$. In addition, $(A, B)=(B, A)^{*}$ and $(A, B+\lambda C)=(A, B)+\lambda(A, C)$ for any complex number $\lambda$. Thus, $(A, B)$ is a positive-definite inner product. It satisfies

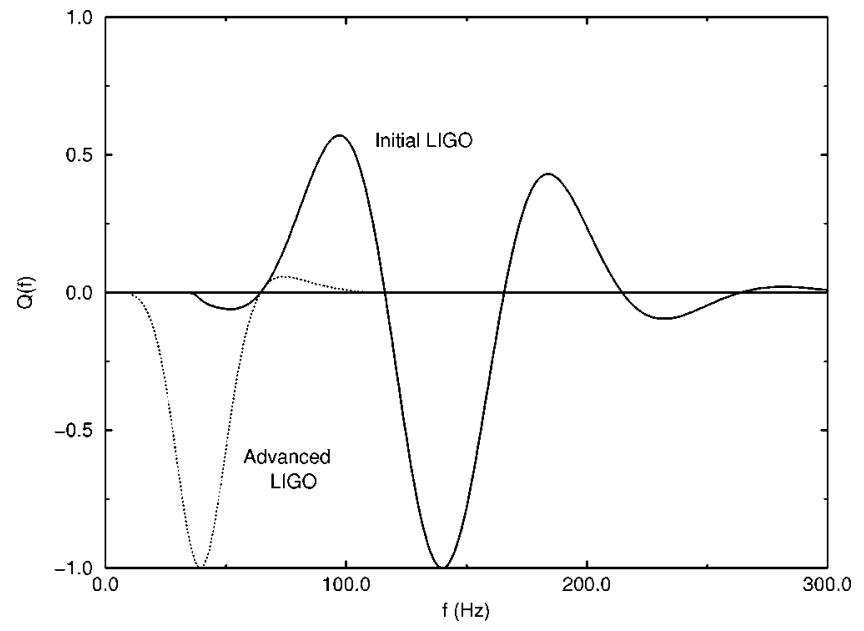

FIG. 4. Optimal filter functions $\widetilde{Q}(f)$ for the initial and advanced LIGO detector pairs, for a stochastic background having a constant frequency spectrum $\Omega_{\mathrm{gw}}(f)=\Omega_{0}$. Both filters are normalized to have maximum magnitude equal to unity. 
all of the properties of an ordinary dot product of vectors in three-dimensional Euclidean space.

In terms of this inner product, the mean value $\mu$ and variance $\sigma^{2}$ can be written as

$$
\begin{aligned}
\mu & =\frac{3 H_{0}^{2}}{20 \pi^{2}} T\left(\widetilde{Q}, \frac{\gamma(|f|) \Omega_{\mathrm{gw}}(|f|)}{|f|^{3} P_{1}(|f|) P_{2}(|f|)}\right), \\
\sigma^{2} & \approx \frac{T}{4}(\widetilde{Q}, \widetilde{Q}) .
\end{aligned}
$$

The problem is to choose $\widetilde{Q}(f)$ so that it maximizes the signal-to-noise ratio (3.51) or equivalently, the squared signal-to-noise ratio

$$
\mathrm{SNR}^{2}=\frac{\mu^{2}}{\sigma^{2}} \approx\left(\frac{3 H_{0}^{2}}{10 \pi^{2}}\right)^{2} T \frac{\left(\widetilde{Q}, \frac{\gamma(|f|) \Omega_{\mathrm{gw}}(|f|)}{|f|^{3} P_{1}(|f|) P_{2}(|f|)}\right)^{2}}{(\widetilde{Q}, \widetilde{Q})} .
$$

But this is trivial. For suppose we are given a fixed threedimensional vector $\vec{A}$, and are asked to find the threedimensional vector $\vec{Q}$ that maximizes the ratio $(\vec{Q} \cdot \vec{A})^{2} / \vec{Q}$ - $\vec{Q}$. Since this ratio is proportional to the squared cosine of the angle between the two vectors, it is maximized by choosing $\vec{Q}$ to point in the same direction as $\vec{A}$. The problem of maximizing Eq. (3.72) is identical. The solution is

$$
\widetilde{Q}(f)=\lambda \frac{\gamma(|f|) \Omega_{\mathrm{gw}}(|f|)}{|f|^{3} P_{1}(|f|) P_{2}(|f|)},
$$

where $\lambda$ is a (real) overall normalization constant.

One of the curious things about expression (3.73) for the optimal filter $\widetilde{Q}(f)$ is that it depends upon the spectrum $\Omega_{\mathrm{gw}}(f)$ of the stochastic gravity-wave background. This is a function that we do not know a priori. ${ }^{17}$ In practice this means that we cannot use a single optimal filter when performing the data analysis; we will need to use a set of such filters. For example, within the bandwidth of interest for the ground-based interferometers, it is reasonable to assume that the spectrum is given by a power law $\Omega_{\mathrm{gw}}(f)=\Omega_{\alpha} f^{\alpha}$ (where $\Omega_{\alpha}=$ const). ${ }^{18}$ We could then construct a set of optimal filters $\widetilde{Q}_{\alpha}(f)$ (say, for $\left.\alpha=-4,-7 / 2, \ldots, 7 / 2,4\right)$ with the overall normalization constants $\lambda_{\alpha}$ chosen so that

$$
\mu=\Omega_{\alpha} T \text {. }
$$

With this choice of normalization, the optimal filter functions $\widetilde{Q}_{\alpha}(f)$ are completely specified by the exponent $\alpha$, the overlap reduction function, and the noise power spectra of the

\footnotetext{
${ }^{17}$ See, however, the discussion surrounding Eqs. (3.58) and (3.59).

${ }^{18}$ The $\alpha$ in $\Omega_{\alpha}$ and $f^{\alpha}$ is just a number; it is not an index label.
}

two detectors. We would then analyze the outputs of the two detectors for each of these filters separately. Figure 4 shows the optimal filter functions (displayed in the frequency domain) for both the initial and advanced LIGO detector pairs, for a stochastic background having a constant frequency spectrum $\Omega_{\mathrm{gw}}(f)=\Omega_{0}$ (i.e., $\alpha=0$ ). ${ }^{19}$ Figure 5 shows these same optimal filter functions displayed in the time domaini.e., as a function of the lag $t-t^{\prime}$.

Having found the optimal choice of filter function $\widetilde{Q}(f)$, it is now straightforward to calculate the signal-to-noise ratio for a given pair of detectors. Substituting Eq. (3.73) into Eq. (3.72) and taking the square root gives

$$
\mathrm{SNR} \approx \frac{3 H_{0}^{2}}{10 \pi^{2}} \sqrt{T}\left[\int_{-\infty}^{\infty} d f \frac{\gamma^{2}(|f|) \Omega_{\mathrm{gw}}^{2}(|f|)}{f^{6} P_{1}(|f|) P_{2}(|f|)}\right]^{1 / 2} .
$$

We will use this result in later sections to calculate signalto-noise ratios and sensitivity levels for different detector pairs, assuming that the stochastic gravity-wave background has a constant frequency spectrum $\Omega_{\mathrm{gw}}(f)=\Omega_{0}$. Tables $\mathrm{I}-\mathrm{V}$ in Sec. VI D contain the results of these calculations.

\section{DETECTION, ESTIMATION, AND SENSITIVITY LEVELS}

Once the detectors have gone "on-line", and are generating data that needs to be analyzed, we will be confronted with the following questions: (i) How do we decide, from the experimental data, if we have detected a stochastic gravitywave signal? (ii) Assuming that a stochastic gravity-wave signal is present, how do we estimate its strength? (iii) Assuming that a stochastic gravity-wave signal is present, what is the minimum value of $\Omega_{0}$ required to detect it $95 \%$ of the time? In this section, we answer these questions, using a frequentist approach to the theory of probability and statistics. $^{20}$

\section{A. Statistical considerations}

When performing a search for a stochastic background of gravitational radiation, it is convenient to break the data set

\footnotetext{
${ }^{19}$ Figures $21-30$ in Sec. VI C show the analogous optimal filter functions for different detector pairs.

${ }^{20}$ There are actually two approaches that one can take when analyzing data: the frequentist (or classical frequency probability) approach, which is adopted in this paper, and the Bayesian (or subjective probability) approach, which is adopted in Refs. [18,19]. Although we will not describe the similarities and differences of these two approaches in any detail in this paper, it is important to emphasize that the frequentist and Bayesian approaches are inequivalent methods of analyzing data. Frequentists and Bayesians ask different questions about data and hypotheses, and consequently obtain different answers and draw different conclusions. In fact, there are certain questions that one can ask and answer in the Bayesian approach that are ill-defined for a frequentist. Interested readers should see Refs. $[18,19]$ for a detailed discussion of the frequentist and Bayesian approaches applied to gravitational-wave data analysis with multiple detectors.
} 


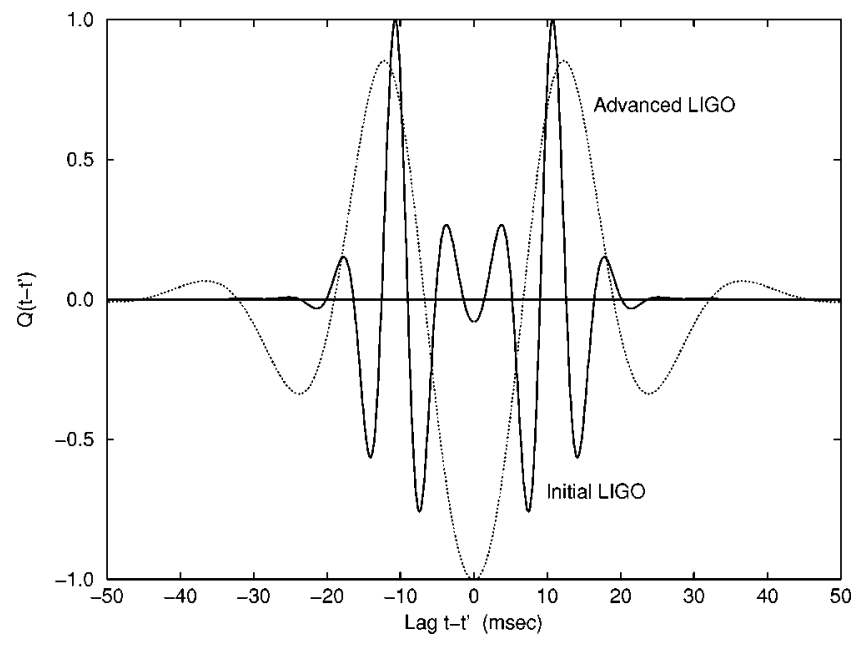

FIG. 5. Optimal filter functions $Q\left(t-t^{\prime}\right)$ for the initial and advanced LIGO detector pairs, for a stochastic background having a constant frequency spectrum $\Omega_{\mathrm{gw}}(f)=\Omega_{0}$. Both filters are normalized to have maximum magnitude equal to unity.

(which might be hours, days, or weeks in length) into shorter stretches of $N$ points, which we can then fast Fourier transform (FFT) and correlate with data from other detectors. Depending on the choice of $N$ and on the sampling rate of the detector, these shorter segments of data will typically last on the order of seconds. ${ }^{21}$ From Eq. (3.50), we see that in a measurement over a single observation period $T \approx 4 \mathrm{sec}$, the signal $S$ is a sum (over $f$ and $f^{\prime}$ ) of approximately 400 statistically independent random variables (products of the Fourier amplitudes of the signals). This is because $\tilde{s}_{1}(f)$ and $\tilde{s}_{2}\left(f^{\prime}\right)$ are correlated only when $\left|f-f^{\prime}\right|<1 / T \approx .25 \mathrm{~Hz}$, and the bandwidth over which the integral in Eq. (3.50) gets its major contribution is $\sim 100 \mathrm{~Hz}$ wide. Thus, by virtue of the central limit theorem, $S$ is well approximated by a Gaussian random variable, provided we are not too far away from the mode of the distribution. Equivalently, the values of $S$ in a set of measurements over statistically independent time intervals (each of length $T$ ) are normally distributed. The mean value of this distribution is $\mu:=\langle S\rangle$, and the variance is $\sigma^{2}:=\left\langle S^{2}\right\rangle-\langle S\rangle^{2}$.

Let $\mathbf{s}:=\left(S_{1}, S_{2}, \ldots, S_{n}\right)$ be a set of such measurements over statistically independent time intervals, each of length $T .^{22}$ We can think of these measurements as $n$ independent samples drawn from a normal distribution having mean $\mu$ and variance $\sigma^{2}$. The set $\mathbf{s}$ represents the outcome of a single

\footnotetext{
${ }^{21}$ For example, for $N=65536=2^{16}$ and a sampling rate of $20 \mathrm{kHz}$, $T=3.2768 \mathrm{sec}$. For the same $N$ and a sampling rate of $16.384 \mathrm{kHz}=2^{14} \mathrm{~Hz}, T=4.0 \mathrm{sec}$. Since the relevant bandwith for stochastic background detection is $f<300 \mathrm{~Hz}$, the data stream could, in principle, be decimated to sampling rates which are substantially smaller-i.e., $1024 \mathrm{~Hz}$.

${ }^{22}$ In order that the measurements be statistically independent, the time intervals should be non-overlapping, and $T$ should be $\gg$ the light travel time $d / c$ between the two detectors. For the LIGO detector pair, this corresponds to $T \gg 10^{-2} \mathrm{sec}$, which is satisfied for $T \approx 4 \mathrm{sec}$.
}

experiment. From these samples, we can construct the sample mean

$$
\hat{\mu}:=\frac{1}{n} \sum_{i=1}^{n} S_{i}
$$

and the sample variance

$$
\hat{\sigma}^{2}:=\frac{1}{n-1} \sum_{i=1}^{n}\left(S_{i}-\hat{\mu}\right)^{2} .
$$

Given the values of these estimators $\hat{\mu}$ and $\hat{\sigma}^{2}$, we would like to decide, in some reliable way, whether or not we have detected a stochastic gravity-wave signal.

To make such a decision, we will apply a standard theorem from the theory of probability and statistics (see, e.g., p. 178 of Ref. [20]). The theorem states that if $\hat{\mu}$ is the sample mean of a set of $n$ independent samples drawn from a normal distribution having mean $\mu$ and variance $\sigma^{2}$, then

$$
t=\frac{\hat{\mu}-\mu}{\hat{\sigma} / \sqrt{n}}
$$

is the value of a random variable having Student's $t$-distribution with parameter $\nu=n-1$. This is the classic Student's $t$-test. It is used to compare the means of two normal distributions that have the same variance, when the variance $\sigma^{2}$ is unknown.

Since tables of Student's $t$-distribution and its associated cumulative probability distribution function can be found in most handbooks on statistics (see, e.g., [21]), we could do all of the remaining calculations in this section in terms of the $t$-distribution. The drawback to this approach, however, is that the $t$-distribution depends on the parameter $\nu=n-1$. This means that all of our results would depend on the number of observations $n$ that constitute a single experiment. For stochastic background searches, this undesirable feature can be avoided by choosing $n$ large enough so that the $t$-distribution and standard normal distribution are virtually indistinguishable. Since a typical total observation time will be on the order of months or years $\left(\sim 10^{7} \mathrm{sec}\right)$, while $T$ (the duration of a single observation period) is typically on the order of seconds, it is no problem to choose $n \sim 10^{3}$ or more. ${ }^{23}$ For such large $n$, Eq. (4.3) can be rewritten as

$$
z \approx \frac{\hat{\mu}-\mu}{\hat{\sigma} / \sqrt{n}}
$$

where $z$ is the value of a random variable having the standard normal distribution-i.e., $z$ is a Gaussian random variable having zero mean and unit variance. Note that the approximation becomes a strict equality if $\hat{\sigma}$ is replaced by its expected value $\sigma$. This is because a linear combination of $n$

\footnotetext{
${ }^{23}$ In fact, even for $n=30$, the $2 \sigma$ values for the $t$-distribution and standard normal distribution differ by less than $5 \%$. As $n \rightarrow \infty$, this difference goes to zero.
} 
Gaussian random variables (e.g., $\hat{\mu}$ ) is also a Gaussian random variable, independent of $n$.

In the calculations that follow, we will want to assign probabilities to different events. From a frequentist point of view, this means that we should perform (or imagine performing) some fixed experiment many, many times. The probability of an event is then defined as the frequency of occurrence of that event, in the limit of an infinite number of repeated, independent experiments.

\section{B. Signal detection}

In order to decide whether or not we have detected a stochastic gravity-wave signal, we need a rule that, given a set of measured data, will select for us one of two alternative hypotheses:

$H_{0}$ : A stochastic gravity-wave signal is absent.

$H_{1}$ : A stochastic gravity-wave signal is present, characterized by some fixed, but unknown, mean value $\mu>0$.

Moreover, we would like this rule to be "optimal" with respect to some chosen set of criteria. This method of decision making, or "hypothesis testing" as it is more formally called, is a well-studied branch of frequentist statistics. As such, we will not go into any of the details here. After making the appropriate definitions, we will simply state the rule that we adopt for our stochastic background searches and explain in what sense it is optimal. Interested readers should see Ref. [22] for a much more thorough discussion of the statistical theory of signal detection.

To begin, let us note that the two hypotheses $H_{0}$ and $H_{1}$ are exhaustive and mutually exclusive-i.e., a stochastic gravity-wave signal is either absent or present. And, if present, it will be characterized by some fixed mean value $\mu$, which is proportional to $\Omega_{0}$ for a stochastic background of gravitational radiation having a constant frequency spectrum $\Omega_{\mathrm{gw}}(f)=\Omega_{0}$. The only requirement is that $\mu>0 . H_{0}$ is a simple hypothesis, since it does not depend on any unknown parameters. $H_{1}$ is a complex (or composite) hypothesis, since it depends on a range of the unknown parameter $\mu$. Explicitly,

$$
H_{1}=\bigcup_{n=0}^{\infty} H_{n d \mu, d \mu}
$$

where $H_{\mu, d \mu}$ is the hypothesis that a stochastic gravity-wave signal is present, characterized by a fixed mean value lying in the range $(\mu, \mu+d \mu]$.

As before, let $\mathbf{s}:=\left(S_{1}, S_{2}, \ldots, S_{n}\right)$ be a set of $n$ statistically independent measurements of the cross-correlation signal $S$. Because of the noise intrinsic to the detectors and errors inherent to the measurement process, the outcome of an experiment $\mathbf{s}$ is a random variable. It is described statistically by the probability density functions:

$p(\mathbf{s} \mid 0)$ : Probability density function for the outcome of an experiment to be $\mathbf{s}$, given that a stochastic gravity-wave signal is absent.

$p(\mathbf{s} \mid \mu)$ : Probability density function for the outcome of an experiment to be $\mathbf{s}$, given that a stochastic gravity-wave signal is present, characterized by the fixed mean value $\mu$ $>0$.

From Eq. (4.4), it follows that

$$
\begin{aligned}
& p(\mathbf{s} \mid 0)=\left(2 \pi \hat{\sigma}^{2}\right)^{-n / 2} \exp \left[-\sum_{i=1}^{n} \frac{S_{i}^{2}}{2 \hat{\sigma}^{2}}\right], \\
& p(\mathbf{s} \mid \mu)=\left(2 \pi \hat{\sigma}^{2}\right)^{-n / 2} \exp \left[-\sum_{i=1}^{n} \frac{\left(S_{i}-\mu\right)^{2}}{2 \hat{\sigma}^{2}}\right] .
\end{aligned}
$$

The fact that we can use the sample variance $\hat{\sigma}^{2}$ (instead of the true variance $\sigma^{2}$ ) on the right-hand sides of Eqs. (4.6) and (4.7) follows from the large $n$ approximation that we used to obtain Eq. (4.4).

A decision rule that, given the outcome of an experiment, selects for us either $H_{0}$ or $H_{1}$ is equivalent to a division of the space of all possible experimental outcomes into two disjoint regions $R_{0}$ and $R_{1}$ : If $\mathbf{s} \in R_{0}$, then $H_{0}$ is chosen; if $\mathbf{s} \in R_{1}$, then $H_{1}$ is chosen. The success and failure of such a rule are characterized by two types of errors: A type $I$ (or false alarm) error occurs when the decision rule chooses $H_{1}$ when $H_{0}$ is really true. A type II (or false dismissal) error occurs when the decision rule chooses $H_{0}$ when $H_{1}$ is really true. In terms of $p(\mathbf{s} \mid 0), p(\mathbf{s} \mid \mu), R_{0}$, and $R_{1}$, we have

$$
\begin{gathered}
\alpha \equiv \text { false alarm rate }:=\int_{R_{1}} d \mathbf{s} p(\mathbf{s} \mid 0), \\
\beta(\mu) \equiv \text { false dismissal rate }:=\int_{R_{0}} d \mathbf{s} p(\mathbf{s} \mid \mu) .
\end{gathered}
$$

Note that, for the complex hypothesis $H_{1}$, the false dismissal rate is actually a function of the mean value $\mu>0$. Note also that $1-\alpha$ is the fraction of experimental outcomes that the decision rule correctly identifies the absence (not presence) of a stochastic gravity-wave signal. If we want to talk about detection, we should evaluate

$$
\gamma(\mu) \equiv \text { detection rate }:=1-\beta(\mu),
$$

which is the fraction of experimental outcomes that the decision rule correctly identifies the presence of a stochastic gravity-wave signal, characterized by the fixed mean value $\mu>0 .{ }^{24}$

In order for the decision rule to choose the regions $R_{0}$ and $R_{1}$ in an "optimal" way, we must first select some set of criteria with respect to which "optimal" can be defined. For stochastic background searches, where one does not know $a$ priori the "costs" that one should associate with false alarm and false dismissal errors, it is reasonable to choose a decision rule that minimizes the false dismissal rate $\beta(\mu)$ for a fixed value of the false alarm rate $\alpha$. Equivalently, one chooses a decision rule that maximizes the probability of

\footnotetext{
${ }^{24}$ The detection rate $\gamma(\mu)$ should not be confused with the overlap reduction function $\gamma(f)$, which was defined in Sec. III B.
} 
detecting a stochastic gravity-wave signal, while keeping the false alarm rate fixed. This decision criterion is known in the literature as the Neyman-Pearson criterion. In general, for a complex hypothesis, the Neyman-Pearson criterion yields regions $R_{0}$ and $R_{1}$ that depend on the unknown parameter $\mu$. But for this case, where $H_{1}$ involves all parameter values $\mu>0, R_{0}$ and $R_{1}$ are actually independent of $\mu .{ }^{25}$ Without going into details here, let us simply state the result: Namely, in the context of stochastic background searches as described above, the Neyman-Pearson criterion is satisfied if, given the outcome of an experiment $\mathbf{s}$, we form the estimators $\hat{\mu}$ and $\hat{\sigma}^{2}$ according to Eqs. (4.1) and (4.2), and then

$$
\begin{aligned}
& \text { choose } H_{0} \text { if } \hat{\mu}<z_{\alpha} \hat{\sigma} / \sqrt{n}, \\
& \text { choose } H_{1} \text { if } \hat{\mu} \geqslant z_{\alpha} \hat{\sigma} / \sqrt{n} .
\end{aligned}
$$

Here $z_{\alpha}$ is that value of the random variable $z$ for which the area under the standard normal distribution to its right is equal to $\alpha$. (See Fig. 6.) In terms of the complementary error function

$$
\operatorname{erfc}(z):=\frac{2}{\sqrt{\pi}} \int_{z}^{\infty} d x e^{-x^{2}}
$$

(see Fig. 7),

$$
z_{\alpha}=\sqrt{2} \operatorname{erfc}^{-1}(2 \alpha)
$$

where $\operatorname{erfc}^{-1}$ denotes the inverse of erfc. Since $\hat{\mu}$ and $\hat{\sigma}$ are functions of the outcome of the experiment $\mathbf{s}$, the inequalities $\hat{\mu}<z_{\alpha} \hat{\sigma} / \sqrt{n}$ and $\hat{\mu} \geqslant z_{\alpha} \hat{\sigma} / \sqrt{n}$ define $R_{0}$ and $R_{1}$. Note also that the decision rule can be restated as

$$
\begin{aligned}
& \text { choose } H_{0} \text { if } \sqrt{n} \widehat{\mathrm{SNR}}<z_{\alpha}, \\
& \text { choose } H_{1} \text { if } \sqrt{n} \widehat{\mathrm{SNR}} \geqslant z_{\alpha},
\end{aligned}
$$

where

$$
\sqrt{n} \widehat{\mathrm{SNR}}:=\sqrt{n} \frac{\hat{\mu}}{\hat{\sigma}}
$$

is the measured signal-to-noise ratio after $n$ observation periods. The fact that the signal-to-noise ratio enters the above inequalities is one of the main reasons why we paid so much attention to evaluating it in Sec. III.

\footnotetext{
${ }^{25}$ See pp. $152-153$ of Ref. [22] for more details.
}

Given the above decision rule for signal detection, we can now calculate the false alarm and false dismissal rates defined by Eqs. (4.8) and (4.9). First, for the false alarm rate, one finds

$$
\begin{aligned}
\alpha & =\operatorname{Prob}\left(\hat{\mu} \geqslant z_{\alpha} \hat{\sigma} / \sqrt{n} \mid \mu=0\right) \\
& =\operatorname{Prob}\left(z \geqslant z_{\alpha}\right) \\
& =\frac{1}{2} \operatorname{erfc}\left(\frac{z_{\alpha}}{\sqrt{2}}\right)
\end{aligned}
$$

where we used Eq. (4.4) with $\mu=0$ to obtain the second equality. Thus, our two uses of the symbol $\alpha$ (for the false alarm rate and for the area under the standard normal distribution to the right of $z_{\alpha}$ ) are consistent.

Second, for the false dismissal rate,

$$
\begin{aligned}
\beta(\mu) & =\operatorname{Prob}\left(\hat{\mu}<z_{\alpha} \hat{\sigma} / \sqrt{n} \mid \mu>0 \text { fixed }\right) \\
& =\operatorname{Prob}\left(z<z_{\alpha}-\sqrt{n} \mu / \hat{\sigma}\right) \\
& =1-\frac{1}{2} \operatorname{erfc}\left(\frac{z_{\alpha}-\sqrt{n} \mu / \hat{\sigma}}{\sqrt{2}}\right)
\end{aligned}
$$

where we again used Eq. (4.4) (but this time with $\mu>0$ ) to obtain the second equality. A graph of the detection rate $\gamma(\mu):=1-\beta(\mu)$ is shown in Fig. 8. From this graph, we see that the detection rate $\gamma(\mu)$ approaches $\alpha$ as $\mu \rightarrow 0$; it approaches 1 as $\mu \rightarrow \infty$. Also, the detection rate equals 0.50 for $\mu=z_{\alpha} \hat{\sigma} / \sqrt{n}$ (or, equivalently, for $\sqrt{n} \mu / \hat{\sigma}=z_{\alpha}$ ). Thus, if we replace $\hat{\sigma}$ with its expected value $\sigma$, we see that the detection rate is only $50 \%$ for a signal having a theoretical signal-to-noise ratio after $n$ observation periods equal to $z_{\alpha}$.

It is also interesting to note that the Neyman-Pearson detection criterion, when applied to stochastic background searches, is equivalent to the maximum-likelihood detection criterion [22]. In other words, we will obtain the same decision rule given above if we first construct the likelihood ratio

$$
\Lambda(\mathbf{s} \mid \mu):=\frac{p(\mathbf{s} \mid \mu)}{p(\mathbf{s} \mid 0)}
$$

maximize $\Lambda(\mathbf{s} \mid \mu)$ with respect to variations of the parameter $\mu>0$,

$$
\Lambda_{\max }(\mathbf{s}):=\max _{\mu>0} \Lambda(\mathbf{s} \mid \mu)
$$

and then divide the space of all possible experimental outcomes $\mathbf{S}$ into two regions $R_{0}$ and $R_{1}$ according to the rule

$$
\begin{aligned}
& \text { choose } H_{0} \text { if } \Lambda_{\max }(\mathbf{s})<\Lambda_{0}, \\
& \text { choose } H_{1} \text { if } \Lambda_{\max }(\mathbf{s}) \geqslant \Lambda_{0},
\end{aligned}
$$

where $\Lambda_{0}$ is chosen so that the false alarm rate equals $\alpha$. Explicitly, from Eqs. (4.6) and (4.7), it follows that 


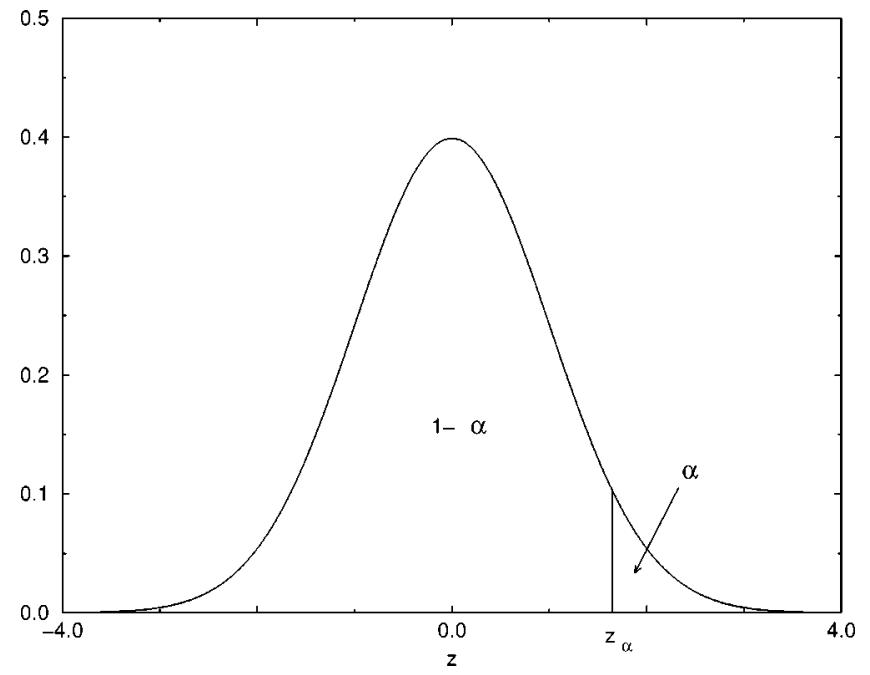

FIG. 6. The standard normal probability distribution for a Gaussian random variable having zero mean and unit variance. $z_{\alpha}$ is that value of $z$ for which the area under the standard normal distribution to its $\overline{r i g h t}$ is equal to $\alpha$. Typical values for $1-\alpha$ are 0.90 , 0.95 , and 0.99 . The corresponding values for $z_{\alpha}$ are $z_{0.10}$ $=1.28, z_{0.05}=1.65$, and $z_{0.01}=2.33$. These are the threshold values appropriate for the one-sided test described in the text.

$$
\Lambda(\mathbf{s} \mid \mu)=\exp \left[\frac{n \mu}{\hat{\sigma}^{2}}\left(\frac{1}{n_{i=1}} \sum_{i}-\frac{1}{2} \mu\right)\right]=\exp \left[\frac{n \mu}{\hat{\sigma}^{2}}\left(\hat{\mu}-\frac{1}{2} \mu\right)\right]
$$

$\Lambda(\mathbf{s} \mid \mu)$ is maximized when $\mu$ equals the sample mean $\hat{\mu}:{ }^{26}$

$$
\Lambda_{\max }(\mathbf{s})=\Lambda(\mathbf{s} \mid \hat{\mu})=\exp \left[\frac{1}{2} \frac{n \hat{\mu}^{2}}{\hat{\sigma}^{2}}\right]
$$

The decision surface $\Lambda_{0}$ is obtained by setting $\hat{\mu}$ equal to the threshold value $z_{\alpha} \hat{\sigma} / \sqrt{n}$ :

$$
\Lambda_{0}=\Lambda_{\max }\left(z_{\alpha} \hat{\sigma} / \sqrt{n}\right)=\exp \left[\frac{1}{2} z_{\alpha}^{2}\right]
$$

\section{Parameter estimation}

Assuming that a stochastic gravity-wave signal is present, characterized by some fixed, but unknown, mean value $\mu$ $>0$, parameter estimation attempts to answer the question, what is the value of $\mu$ ? It does this by first constructing the sample mean $\hat{\mu}$ and sample variance $\hat{\sigma}^{2}$ of a set $\mathbf{s}$ $:=\left(S_{1}, S_{2}, \ldots, S_{n}\right)$ of $n$ statistically independent measurements of the cross-correlation signal $S$, as described in Sec. IV A. From Eq. (4.4), we then know that

$$
z \approx \frac{\hat{\mu}-\mu}{\hat{\sigma} / \sqrt{n}}
$$

\footnotetext{
${ }^{26}$ The sample mean $\hat{\mu}$ is said to be the maximum-likelihood estimator for this problem.
}

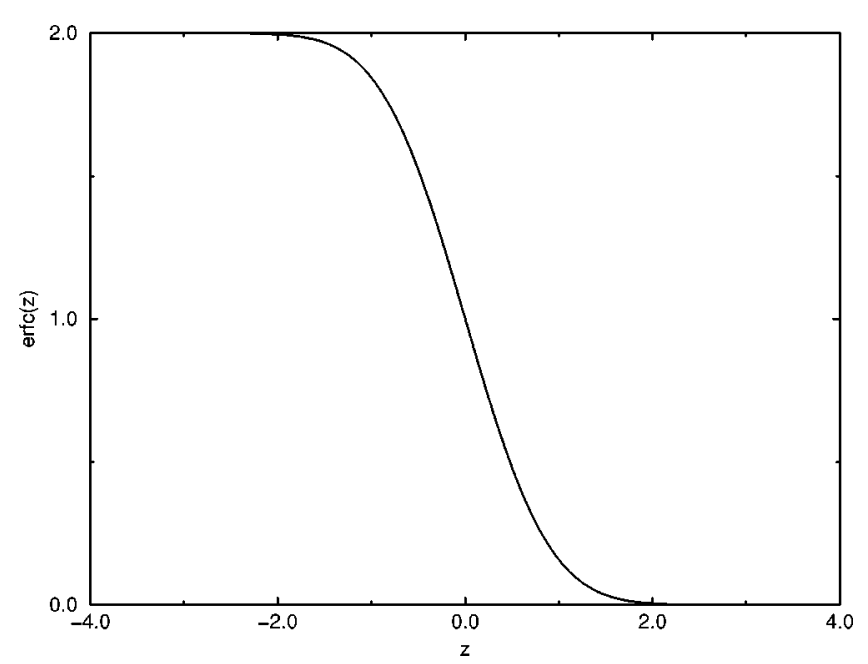

FIG. 7. The complementary error function.

is the value of a Gaussian random variable having zero mean and unit variance. Thus, if $z_{\alpha / 2}$ is that value of the random variable $z$ for which the area under the standard normal distribution to its right is equal to $\alpha / 2$ (see Fig. 9), then (1 $-\alpha) \times 100 \%$ of the time

$$
-z_{\alpha / 2}<z<z_{\alpha / 2}
$$

or, equivalently,

$$
\hat{\mu}-z_{\alpha / 2} \hat{\sigma} / \sqrt{n}<\mu<\hat{\mu}+z_{\alpha / 2} \hat{\sigma} / \sqrt{n} \text {. }
$$

Said another way, in an ensemble of observations of the same stochastic background, a fraction $1-\alpha$ of the intervals

$$
I_{\alpha}:=\left[\hat{\mu}-z_{\alpha / 2} \hat{\sigma} / \sqrt{n}, \hat{\mu}+z_{\alpha / 2} \hat{\sigma} / \sqrt{n}\right]
$$

constructed from the measured data, will contain the value of the true mean $\mu$. Equivalently, $\alpha$ is the fraction of intervals $I_{\alpha}$ that fail to contain the value of the true mean $\mu$. Of course, given the outcome of a single experiment $\mathbf{s}$, the interval $I_{\alpha}$ either contains or does not contain the value of $\mu$. And the value of $\mu$, if it is contained in $I_{\alpha}$, need not be any closer to the center of the interval than to either of its edges. Thus, the confidence that one associates with the above estimation procedure is not equivalent to our degree of belief that the true mean $\mu$ lies within a given interval. (This is a Bayesian interpretation of probability.) Rather, it is the fraction of experimental outcomes that our estimation procedure will produce an interval that contains the true mean $\mu$, in the limit of an infinite number of repeated, independent experiments.

\section{Sensitivity levels}

Let us assume once again that a stochastic gravity-wave signal is present, characterized by some fixed, but unknown, mean value $\mu>0$. Then it is reasonable to ask what is the minimum value of $\mu$ required so that our decision rule correctly identifies the presence of a signal at least $\gamma \times 100 \%$ of the time? 


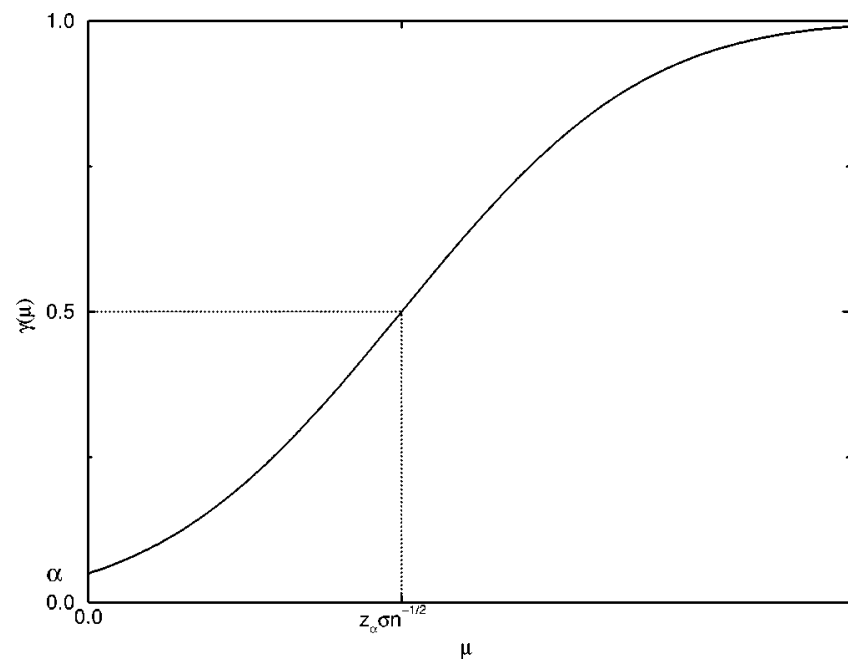

FIG. 8. The detection rate $\gamma(\mu):=1-\beta(\mu)$ plotted as a function of the mean value $\mu$.

The answer to this question can be obtained by applying the results of Sec. IV B. Namely, we require that the detection rate $\gamma(\mu):=1-\beta(\mu)$ be greater than or equal to the desired rate $\gamma$, and then solve the resulting inequality for $\mu$. Explicitly, from Eq. (4.21), it follows that

$$
1-\beta(\mu)=\frac{1}{2} \operatorname{erfc}\left(\frac{z_{\alpha}-\sqrt{n} \mu / \hat{\sigma}}{\sqrt{2}}\right) \geqslant \gamma
$$

or, equivalently,

$$
z_{\alpha}-\sqrt{n} \mu / \sigma \leqslant \sqrt{2} \operatorname{erfc}^{-1}(2 \gamma)
$$

where we replaced the estimator $\hat{\sigma}$ by its expected value $\sigma$ to obtain the LHS of the above equation. Thus,

$$
\begin{aligned}
\mu & \geqslant \frac{\sigma}{\sqrt{n}}\left[z_{\alpha}-\sqrt{2} \operatorname{erfc}^{-1}(2 \gamma)\right] \\
& =\frac{\sigma}{\sqrt{n}} \sqrt{2}\left[\operatorname{erfc}^{-1}(2 \alpha)-\operatorname{erfc}^{-1}(2 \gamma)\right] .
\end{aligned}
$$

Equivalently,

$$
\sqrt{n} \mathrm{SNR} \geqslant \sqrt{2}\left[\operatorname{erfc}^{-1}(2 \alpha)-\operatorname{erfc}^{-1}(2 \gamma)\right]
$$

where $\sqrt{n} \mathrm{SNR}:=\sqrt{n} \mu / \sigma$ is the theoretical signal-to-noise ratio after $n$ observation periods. Note that the right-hand sides of Eqs. (4.35) and (4.36) depend on both $\alpha$ and $\gamma \cdot{ }^{27}$ This means that to calculate the minimum value of $\mu$ (or the minimum signal-to-noise ratio), we must specify the desired detection rate $\gamma$ in addition to the false alarm rate $\alpha$. In the past (see, e.g., Refs. $[7,8]$ ), physicists have only specified the false alarm rate $\alpha$. It seems that they have mistakenly assumed that the probability of correctly identifying the pres-

\footnotetext{
${ }^{27}$ The dependence on $\alpha$ is via the threshold value $z_{\alpha}$, which defines the decision rule (4.14).
}

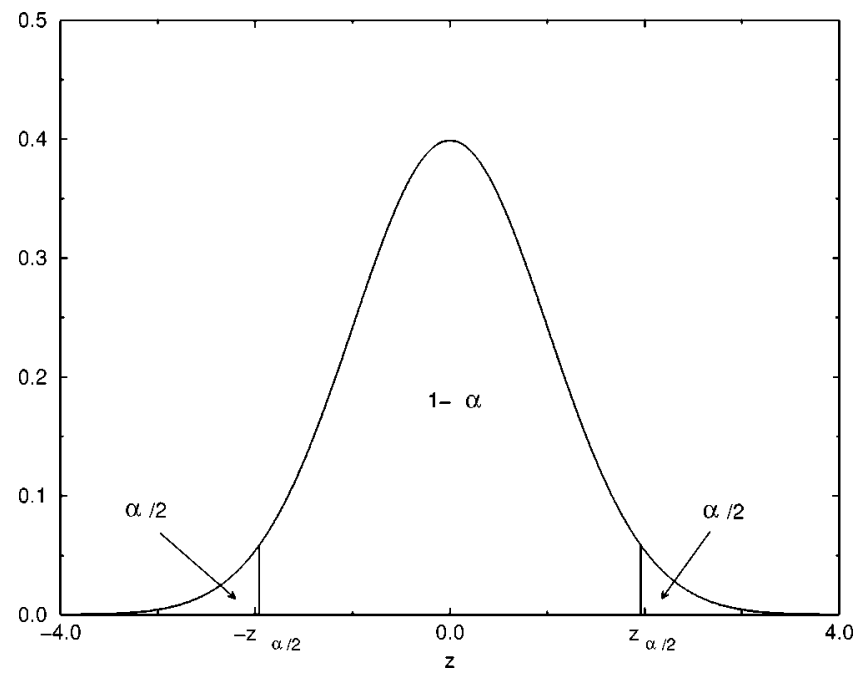

FIG. 9. The standard normal probability distribution for a Gaussian random variable having zero mean and unit variance. $z_{\alpha / 2}$ is that value of $z$ for which the area under the standard normal distribution to its right is equal to $\alpha / 2$. The area under the standard normal distribution between $-z_{\alpha / 2}$ and $z_{\alpha / 2}$ is thus $1-\alpha$. Typical values for $1-\alpha$ are $0.90,0.95$, and 0.99 . The corresponding values for $z_{\alpha / 2}$ are $z_{0.05}=1.65, z_{0.025}=1.96$, and $z_{0.005}=2.58$.

ence of a signal was $1-\alpha$. But as mentioned in Sec. IV B, $1-\alpha$ is the probability that the decision rule correctly identifies the absence of a signal. To talk about detection (and the associated minimum value of $\mu$ ), one needs to include the additional parameter $\gamma$.

For stochastic background searches, we can rewrite Eq. (4.35) in terms of $\Omega_{0}, \gamma(f)$, and the noise power spectra $P_{1}(f)$ and $P_{2}(f)$ of the two detectors:

$$
\begin{aligned}
\Omega_{0} \geqslant & \frac{1}{\sqrt{T_{\text {tot }}}} \frac{10 \pi^{2}}{3 H_{0}^{2}}\left[\int_{-\infty}^{\infty} d f \frac{\gamma^{2}(|f|)}{f^{6} P_{1}(|f|) P_{2}(|f|)}\right]^{-1 / 2} \\
& \times \sqrt{2}\left[\operatorname{erfc}^{-1}(2 \alpha)-\operatorname{erfc}^{-1}(2 \gamma)\right]
\end{aligned}
$$

where $T_{\text {tot }}:=n T$ is the duration of a single experiment $\mathbf{s}$. This result follows from Eq. (3.75) for a stochastic background of gravitational radiation having a constant frequency spectrum $\Omega_{\mathrm{gw}}(f)=\Omega_{0}$. Let us now evaluate the minimum value of $\Omega_{0}$ for 4 months of observation (i.e., $T_{\text {tot }}$ $=10^{7} \mathrm{sec}$ ), for a false alarm rate $\alpha=0.05$, and for a detection rate $\gamma=0.95$. Denoting the solution by $\Omega_{0}^{95 \%, 5 \%}$, we find $^{28}$

(i) $\Omega_{0}^{95 \%, 5 \%} h_{100}^{2}=5.74 \times 10^{-6}$ for the initial LIGO detector pair,

(ii) $\Omega_{0}^{95 \%, 5 \%} h_{100}^{2}=5.68 \times 10^{-11}$ for the advanced LIGO detector pair.

\footnotetext{
${ }^{28}$ There is nothing special about the choice $\alpha=0.05$ and $\gamma$ $=0.95$. For example, $\alpha$ and $\gamma$ need not sum to 1 . We could equally well have chosen $\alpha=0.10$ and $\gamma=0.95$, and calculated a different minimum value $\Omega_{0}^{95 \%, 10 \%}$.
} 
Note, however, that these values disagree with those quoted in the literature (see, e.g., Refs $[7,8]$ ). In Refs. [7,8], the minimum value of $\Omega_{0}$ is determined by the equation

$$
\begin{aligned}
\Omega_{0}= & \frac{1}{\sqrt{T_{\text {tot }}}} \frac{10 \pi^{2}}{3 H_{0}^{2}}\left[\int_{-\infty}^{\infty} d f \frac{\gamma^{2}(|f|)}{f^{6} P_{1}(|f|) P_{2}(|f|)}\right]^{-1 / 2} \\
& \times \sqrt{2} \operatorname{erfc}^{-1}(\alpha) .
\end{aligned}
$$

There are two mistakes: First, the argument of the inverse complementary error function is $\alpha$ instead of $2 \alpha$. But this is appropriate for a two-sided test, which would be correct if the mean value $\mu$ could be either positive or negative. Second, and more importantly, there is no term proportional to $\operatorname{erfc}^{-1}(2 \gamma)$. As mentioned above, it seems that the authors mistakenly assumed that the probability of correctly identifying the presence of a signal was $1-\alpha$. Even the $\alpha$ $=0.10, \gamma=0.90$ value of Eq. (4.37) does not agree with the values of $\Omega_{0}^{90 \%}$ quoted in Refs. $[7,8]$. Thus, by $\Omega_{0}^{90 \%}$ the authors do not mean the minimum value of $\Omega_{0}$ for a false alarm rate equal to $10 \%$ and a detection rate equal to $90 \%$.

Alternatively, the absence of the term $\operatorname{erfc}^{-1}(2 \gamma)$ from Eq. (4.38) is equivalent to calculating the minimum value of $\Omega_{0}$ for a detection rate $\gamma=0.50$. This is because $\operatorname{erfc}^{-1}(1)$ $=0$. Thus, the values of $\Omega_{0}^{90 \%}$ quoted in Refs. [7,8] are for a false alarm rate equal to $5 \%$ and a detection rate equal to $50 \%$ - not $90 \%$ as they claim. This is why the minimum values of $\Omega_{0}$ quoted in those papers are smaller than those found here.

Table I in Sec. VI D contains theoretical signal-to-noise ratios after 4 months of observation, for a stochastic background having a constant frequency spectrum $\Omega_{\mathrm{gw}}(f)=\Omega_{0}$ $=6 \times 10^{6} h_{100}^{-2}$.

Table II in Sec. VI D contains minimum values of $\Omega_{0} h_{100}^{2}$ for 4 months of observation, for a false alarm rate equal to $5 \%$, and for a detection rate equal to $95 \%$.

\section{E. Summary}

We started this section by asking a series of questions. To conclude, we summarize the answers obtained above, and address a couple of other related issues.

(i) How do we decide, from the experimental data, if we have detected a stochastic gravity-wave signal?

Answer: We compare the measured signal-to-noise ratio after $n$ observation periods to the threshold value $z_{\alpha}$. If $\sqrt{n} \widehat{\mathrm{SNR}}<z_{\alpha}$, we conclude that a stochastic gravity-wave signal is absent. If $\sqrt{n} \widehat{\mathrm{SNR}} \geqslant z_{\alpha}$, we conclude that a stochastic gravity-wave signal is present, characterized by some fixed, but unknown, mean value $\mu>0$.

Note, however, that we can never conclude, with $100 \%$ confidence, that a stochastic gravity-wave signal is absent or present. Our decision rule leads us to infer one of these two possibilities, but the rule is not perfect. The false alarm rate $\alpha$ and false dismissal rates $\beta(\mu)$ [defined by Eqs. (4.8) and (4.9)] are the error rates associated with the rule. Thus, claims about the absence or presence of a stochastic gravity- wave signal should always be made with these error rates in mind.

Moreover, one has to be very careful about trying to define "termination" criteria. For example, it would be misleading to try to terminate an experiment by correlating the outputs of two gravity-wave detectors until the measured signal-to-noise ratio for the total observation period exceeds some threshold value $z_{\alpha}$. One can show that the false alarm rate associated with such a rule is $100 \%$. In other words, the conclusion drawn from such an experiment would always be that a stochastic gravity-wave signal is present, even in the absence of a signal. Noises intrinsic to the detectors and errors inherent to the measurement process are sufficient to guarantee that the measured signal-to-noise ratio for the total observation period will eventually exceed any $z_{\alpha}$.

If, however, the value of the threshold level increases with observation time in an appropriate manner, then one can define termination criteria that have false alarm rates less than $100 \%$. A famous theorem of probability and statistics, called the law of the iterated logarithm [23], states that if $S_{1}, S_{2}, \ldots$ are statistically independent and identically distributed random variables with zero mean and finite variance $\sigma^{2}$, then $^{29}$

$$
\operatorname{Prob}\left(\limsup _{n \rightarrow \infty} \frac{S_{1}+S_{2}+\cdots+S_{n}}{\sigma \sqrt{2 n \log [\log (n)]}}=1\right)=1 .
$$

This means that if $\lambda<1$, there is unit probability that

$$
\frac{S_{1}+S_{2}+\cdots+S_{n}}{\sigma \sqrt{2 n \log [\log (n)]}}>\lambda
$$

for infinitely many $n$. If $\lambda>1$, there is unit probability that Eq. (4.40) holds for only finitely many $n$. In terms of the measured signal-to-noise ratio after $n$ observation periods, Eq. (4.40) can be rewritten as

$$
\sqrt{n} \widehat{\mathrm{SNR}}>\lambda \sqrt{2 \log [\log (n)]},
$$

where we have replaced $\hat{\sigma}$ in the definition of $\widehat{\mathrm{SNR}}$ by its expected value $\sigma$. Thus, if we want to define a termination criterion with a false alarm rate less than $100 \%$, we should compare $\sqrt{n} \widehat{\mathrm{SNR}}$ with threshold levels $t_{n}$ satisfying

$$
\lim _{n \rightarrow \infty} \frac{t_{n}}{\sqrt{\log [\log (n)]}}=\infty
$$

This is consistent with the claim made in the previous paragraph that constant threshold levels $t_{n}=z_{\alpha}$ will always be exceeded for some $n$. Unfortunately, we have not been able to write down a simple analytic expression for the false alarm rate, for arbitrary threshold levels $t_{n}$ satisfying Eq.

\footnotetext{
${ }^{29}$ The $\lim \sup _{n \rightarrow \infty}$ of a sequence $x_{1}, x_{2}, \ldots$ is defined as follows: Let $a_{m}$ equal the least upper bound of the subsequence $x_{m}, x_{m+1}, \ldots$. (Note that $a_{1} \geqslant a_{2} \geqslant \ldots$.) Then $\lim \sup _{n \rightarrow \infty} x_{n}$ $:=\lim _{m \rightarrow \infty} a_{m}$.
} 
(4.42). The probability that $\sqrt{n} \widehat{\mathrm{SNR}} \geqslant t_{n}$ for some $n$ involves integrals of products of the Gaussian probability density function with the complementary error function.

(ii) Assuming that a stochastic gravity-wave signal is present, how do we estimate its strength?

Answer: Assuming that a stochastic gravity-wave signal is present, characterized by some fixed, but unknown, mean value $\mu>0$, we estimate $\mu$ by constructing the interval

$$
I_{\alpha}:=\left[\hat{\mu}-z_{\alpha / 2} \hat{\sigma} / \sqrt{n}, \hat{\mu}+z_{\alpha / 2} \hat{\sigma} / \sqrt{n}\right]
$$

In an ensemble of observations of the same stochastic background, $1-\alpha$ is the fraction of intervals $I_{\alpha}$ constructed in this way that contain the value of the true mean $\mu$.

We should emphasize that parameter estimation assumes the presence of a signal. First, as mentioned above, we can never be $100 \%$ certain that a stochastic gravity-wave signal is present. Second, it does not make any sense to try to estimate the parameters of something that we assume does not exist.

(iii) Assuming that a stochastic gravity-wave signal is present, what is the minimum value of $\Omega_{0}$ required to detect it $\gamma \times 100 \%$ of time?

Answer: Assuming that a stochastic gravity-wave signal is present, characterized by some fixed, but unknown, mean value $\mu>0$, the minimum value of $\Omega_{0}$, for an observation time $T_{\text {tot }}$, false alarm rate $\alpha$, and detection rate $\gamma$, is given by

$$
\begin{aligned}
\Omega_{0}= & \frac{1}{\sqrt{T_{\text {tot }}}} \frac{10 \pi^{2}}{3 H_{0}^{2}}\left[\int_{-\infty}^{\infty} d f \frac{\gamma^{2}(|f|)}{f^{6} P_{1}(|f|) P_{2}(|f|)}\right]^{-1 / 2} \\
& \times \sqrt{2}\left[\operatorname{erfc}^{-1}(2 \alpha)-\operatorname{erfc}^{-1}(2 \gamma)\right]
\end{aligned}
$$

For fixed $\alpha$ and $\gamma$, the factor of $T_{\text {tot }}^{-1 / 2}$ implies that the minimum value of $\Omega_{0}$ decreases with increasing observation time. Thus, the sensitivity improves as the total observation time increases. This means that as $T_{\text {tot }}$ increases we can put a tighter "upper limit" on the value of $\Omega_{0}$ for a stochastic gravity-wave signal that we will falsely dismiss more than $(1-\gamma) \times 100 \%$ of the time.

Alternatively, for a fixed $\Omega_{0}$ and a fixed false alarm rate $\alpha$, the factor of $T_{\text {tot }}^{-1 / 2}$ implies that the false dismissmal rate $1-\gamma$, for a stochastic gravity-wave signal having a strength equal to $\Omega_{0}$, decreases with increasing observation time. For example, suppose that a particular theory-such as cosmic strings-predicts a value of $\Omega_{0}=10^{-7}$. Moreover, suppose that, in successive years of observation, we fail to detect the presence of a signal at this level of sensitivity. Then we can still say that the probability of our falsely dismissing a stochastic gravity-wave signal having a strength equal to $\Omega_{0}$ $=10^{-7}$ has decreased over the course of the observation.

The above upper limit on stochastic background signal strengths [defined by Eq. (4.44)] is different from that obtained by setting the measured signal-to-noise ratio for the total observation period equal to the threshold value $z_{\alpha}$ :

$$
\begin{aligned}
\Omega_{0}= & \frac{1}{\sqrt{T_{\text {tot }}}} \frac{10 \pi^{2}}{3 H_{0}^{2}}\left[\int_{-\infty}^{\infty} d f \frac{\gamma^{2}(|f|)}{f^{6} P_{1}(|f|) P_{2}(|f|)}\right]^{-1 / 2} \\
& \times \sqrt{2} \operatorname{erfc}^{-1}(2 \alpha) .
\end{aligned}
$$

This alternative upper limit is the maximum value of the test statistic leading to the conclusion that a stochastic gravitywave signal is absent. The decision rule-by its very construction-will never allow one to conclude that a stochastic gravity-wave signal is present with an $\Omega_{0}$ less than this upper limit.

The above two definitions of upper limit agree when the detection rate $\gamma=0.50$. This is because $\operatorname{erfc}^{-1}(1)=0$. This means that, if a stochastic gravity-wave signal is present, with an $\Omega_{0}$ less than or equal to this maximum value, then there is more than a $50 \%$ chance of falsely dismissing it. Note also that if we change the argument of the inverse complementary error function from $\alpha$ to $2 \alpha$ in Eq. (4.38), then Eqs. (4.38) and (4.45) agree. Thus, the minimum values of $\Omega_{0}$ quoted in Refs. $[7,8]$ can be interpreted as either the $\gamma=0.50, \alpha=0.05$ upper limit defined by Eq. (4.44) or the $\alpha$ $=0.05$ upper limit defined by Eq. (4.45).

\section{COMPLICATIONS}

In Sec. III, we discussed optimal filtering under the assumptions that the noises intrinsic to the detectors were (i) stationary, (ii) Gaussian, (iii) statistically independent of one another and of the stochastic gravity-wave background, and (iv) much larger in magnitude than the stochastic gravitywave background. In this section, we describe the modifications that are necessary when most of these assumptions are removed. ${ }^{30}$ We will also describe how one can correlate the outputs of 4 or more detectors, and how one can combine data from multiple detector pairs to increase the sensitivity of a stochastic background search.

\section{A. Signal-to-noise ratios for arbitrarily large stochastic backgrounds}

In Sec. III C, we calculated the signal-to-noise ratio for a stochastic background of gravitational radiation, assuming that the noises intrinsic to the detectors were much larger in magnitude than the gravitational strains. Although this assumption is most likely valid, there are at least two reasons why we want to consider stochastic background signals whose magnitudes are comparable to (or larger than) the noise intrinsic to the detectors: (i) Computer simulations for stochastic background searches allow one to "dial-in" arbitrarily large signal strengths. Thus, in order to compare theoretical predictions with the results of computer simulations, we need to be able to analyze the large signal case. (ii) $\mathrm{Fu}-$ ture generations of gravity-wave detectors might have intrinsic detector noise levels comparable to the level of a real

\footnotetext{
${ }^{30} \mathrm{We}$ will always assume that the noise intrinsic to a detector is Gaussian and statistically independent of the gravitational strains.
} 
stochastic background signal. For this case, optimal filtering of large signal data will also be necessary.

To begin, let us recall the main results of Sec. III C. Under the assumption that the noises intrinsic to the detectors were much larger than the gravitational strains, the calculation of the variance $\sigma^{2}:=\left\langle S^{2}\right\rangle-\langle S\rangle^{2}$ simplified considerably. For the large noise case, $\sigma^{2}$ was dominated by the pure detector noise contribution:

$$
\sigma^{2} \approx \frac{T}{4} \int_{-\infty}^{\infty} d f P_{1}(|f|) P_{2}(|f|)|\widetilde{Q}(f)|^{2} .
$$

This is Eq. (3.68) from Sec. III C.

If, however, the magnitude of the stochastic background signal is comparable to the noises intrinsic to the detectors, the calculation of the signal-to-noise ratio $\mathrm{SNR}:=\mu / \sigma$ is more involved. Although the mean value $\mu$ is independent of the relative size of the stochastic background signal and the detector noise, ${ }^{31}$

$$
\mu=\frac{3 H_{0}^{2}}{20 \pi^{2}} T \int_{-\infty}^{\infty} d f|f|^{-3} \Omega_{\mathrm{gw}}(|f|) \gamma(|f|) \widetilde{Q}(f),
$$

the variance $\sigma^{2}$ is not. Explicitly,

$$
\sigma^{2}=\frac{T}{4} \int_{-\infty}^{\infty} d f|\widetilde{Q}(f)|^{2} R(f)
$$

where

$$
\begin{aligned}
R(f):= & {\left[P_{1}(|f|) P_{2}(|f|)+\left(\frac{3 H_{0}^{2}}{10 \pi^{2}}\right) \frac{\Omega_{\mathrm{gw}}(|f|)}{|f|^{3}}\right.} \\
& \times\left(P_{1}(|f|)+P_{2}(|f|)\right)+\left(\frac{3 H_{0}^{2}}{10 \pi^{2}}\right)^{2} \frac{\Omega_{\mathrm{gw}}^{2}(|f|)}{f^{6}} \\
& \left.\times\left(1+\gamma^{2}(|f|)\right)\right] .
\end{aligned}
$$

Note the additional terms that contribute to the variance. Roughly speaking, they can be thought of as two "signal +noise" cross-terms and a "pure signal" variance term. ${ }^{32}$ When $\Omega_{\mathrm{gw}}(|f|)$ is negligible compared to the detector noise power spectra $P_{i}(|f|) \quad(i=1,2)$, Eqs. (5.3) and (5.4) for $\sigma^{2}$ reduce to the "pure noise" variance term (5.1) as they should.

At this stage of the analysis, the filter function $\widetilde{Q}(f)$ in Eqs. (5.2) and (5.3) is arbitrary. For the case of large detector noise, we were able to make an optimal choice for $\widetilde{Q}(f)$, which maximized the signal-to-noise ratio. This was facilitated by introducing an inner product

\footnotetext{
${ }^{31}$ See the discussion surrounding Eqs. (3.52)-(3.57) in Sec. III C.

${ }^{32}$ These are the terms proportional to $\Omega_{\mathrm{gw}}(|f|)$ and $\Omega_{\mathrm{gw}}^{2}(|f|)$, respectively.
}

$$
(A, B):=\int_{-\infty}^{\infty} d f A^{*}(f) B(f) P_{1}(|f|) P_{2}(|f|),
$$

and writing $\mu$ and $\sigma^{2}$ in terms of this inner product. The squared signal-to-noise ratio then took the form

$$
\mathrm{SNR}^{2}=\left(\frac{3 H_{0}^{2}}{10 \pi^{2}}\right)^{2} T \frac{\left(\widetilde{Q}, \frac{\gamma(|f|) \Omega_{\mathrm{gw}}(|f|)}{|f|^{3} P_{1}(|f|) P_{2}(|f|)}\right)^{2}}{(\widetilde{Q}, \widetilde{Q})},
$$

which was maximized by choosing

$$
\widetilde{Q}(f)=\lambda \frac{\gamma(|f|) \Omega_{\mathrm{gw}}(|f|)}{|f|^{3} P_{1}(|f|) P_{2}(|f|)} .
$$

Although $\widetilde{Q}(f)$ depended on $\Omega_{\mathrm{gw}}(f)$, we could construct a set of optimal filters $\widetilde{Q}_{\alpha}(f)$ for stochastic backgrounds having power-law spectra $\Omega_{\mathrm{gw}}(f)=\Omega_{\alpha} f^{\alpha}$ (where $\Omega_{\alpha}=$ const). The proportionality constants $\Omega_{\alpha}$ could always be absorbed into the normalization constants $\lambda_{\alpha}$. The resulting set of optimal filters was then completely specified by the exponent $\alpha$, the overlap reduction function, and the noise power spectra of the two detectors.

For the case where the stochastic background is comparable to the noise intrinsic to the detectors, we can try to do something similar. We can define a new inner product

$$
(A, B):=\int_{-\infty}^{\infty} d f A^{*}(f) B(f) R(f),
$$

where $R(f)$ is given by Eq. (5.4). Although this inner product is more complicated in form than the original inner product (5.5), it is still positive-definite, since $R(f)$ is real and positive. In terms of Eq. (5.8),

$$
\mu=\frac{3 H_{0}^{2}}{20 \pi^{2}} T(\widetilde{Q}, A) \quad \text { and } \quad \sigma^{2}=\frac{T}{4}(\widetilde{Q}, \widetilde{Q}),
$$

where

$$
A(f):=|f|^{-3} \Omega_{\mathrm{gw}}(|f|) \gamma(|f|) R^{-1}(f) .
$$

The squared signal-to-noise ratio is thus

$$
\mathrm{SNR}^{2}=\left(\frac{3 H_{0}^{2}}{10 \pi^{2}}\right)^{2} T \frac{(\widetilde{Q}, A)^{2}}{(\widetilde{Q}, \widetilde{Q})},
$$

which has the same form as Eq. (5.6), although with a much more complicated expression for $A(f)$. But the same argument for maximizing the squared signal-to-noise ratio still goes through. The optimal choice of filter function is

$$
\widetilde{Q}(f)=\lambda A(f),
$$

where $\lambda$ is a (real) overall normalization constant.

But this is where the similarity with the large detector noise case ends, and where the complications start to arise. The main problem is that the optimal filter function $\widetilde{Q}(f)$ has 
a complicated functional dependence on the stochastic gravity-wave spectrum $\Omega_{\mathrm{gw}}(f)$. For the large detector noise case, this problem did not exist. As mentioned earlier, we could always consider stochastic backgrounds having powerlaw spectra $\Omega_{\mathrm{gw}}(f)=\Omega_{\alpha} f^{\alpha}$, and then construct a set of optimal filters $\widetilde{Q}_{\alpha}(f)$ labeled by the different values of $\alpha$. But for the optimal filter function $\widetilde{Q}(f)$ given by Eqs. (5.12), (5.10), and (5.4), this idea of constructing a set of filters labeled by only the power-law exponents $\alpha$ fails. The proportionality constants $\Omega_{\alpha}$ cannot be absorbed into the normalization constants $\lambda_{\alpha}$. For example, if we consider a stochastic background having a constant frequency spectrum $\Omega_{\mathrm{gw}}(f)=\Omega_{0}$ (i.e., $\alpha=0$ ), then

$$
\begin{aligned}
\widetilde{Q}(f)= & \lambda \Omega_{0}|f|^{-3} \gamma(|f|)\left[P_{1}(|f|) P_{2}(|f|)\right. \\
& +\left(\frac{3 H_{0}^{2}}{10 \pi^{2}}\right) \frac{\Omega_{0}}{|f|^{3}}\left(P_{1}(|f|)+P_{2}(|f|)\right) \\
& \left.+\left(\frac{3 H_{0}^{2}}{10 \pi^{2}}\right)^{2} \frac{\Omega_{0}^{2}}{f^{6}}\left(1+\gamma^{2}(|f|)\right)\right]^{-1}
\end{aligned}
$$

Although the factor of $\Omega_{0}$ in the numerator can be absorbed into the normalization constant $\lambda$, the factors of $\Omega_{0}$ and $\Omega_{0}^{2}$ in the denominator cannot. In other words, if we want to construct a set of optimal filters for arbitrarily large stochastic backgrounds having power-law spectra $\Omega_{\mathrm{gw}}(f)=\Omega_{\alpha} f^{\alpha}$, we need to specify the proportionality constants $\Omega_{\alpha}$ in addition to the exponents $\alpha$. The "space of optimal filters" thus becomes a much larger set, parametrized by $\left(\alpha, \Omega_{\alpha}\right)$. Although in principle this poses no problem, in practice it requires a more sophisticated search algorithm; the detector outputs will have to be analyzed for each of the filters $\widetilde{Q}_{\left(\alpha, \Omega_{\alpha}\right)}(f)$ separately.

\section{B. Nonstationary detector noise}

It is not at all uncommon for the power spectra of the noises intrinsic to the detectors to change over the course of time. There will be periods of time when the detectors are relatively "quiet," and other periods of time when the detectors are relatively "noisy." 33 These changes in the power spectra will, in turn, lead to measurements whose statistical properties also change with time. For example, during the quiet periods of detector operation, the measurements ${ }^{(1)} S_{1},{ }^{(1)} S_{2}, \ldots,{ }^{(1)} S_{n_{1}}$ will have an associated variance ${ }^{(1)} \sigma^{2}$ that will be smaller than the variance ${ }^{(2)} \sigma^{2}$ associated with the measurements ${ }^{(2)} S_{1},{ }^{(2)} S_{2}, \ldots,{ }^{(2)} S_{n_{2}}$ taken during the noisy periods. Moreover, if the optimal filter function is normalized [by an appropriate choice of $\lambda$ in Eq.

\footnotetext{
${ }^{33}$ Although the frequency of these more "noisy" periods should decrease as the detectors are gradually improved over the course of months or years, variations in the detector noise power spectra will still inevitably occur.
}

(3.73)] so that the theoretical mean $\mu$ is equal to some fixed value [e.g., $\mu=\Omega_{\alpha} T$ for a stochastic background having a power-law spectrum $\Omega_{\mathrm{gw}}(f)=\Omega_{\alpha} f^{\alpha}$ ], then the quiet periods will have a correspondingly larger signal-to-noise ratio. Thus, a natural question that arises in this context is, can one combine the different sets of measurements, corresponding to the quiet and noisy periods of detector operation, so as to maximize the overall signal-to-noise ratio? The answer to this question is yes, and the proof is sketched below.

In order to handle the most general case, let us consider $m$ different sets of measurements

$$
{ }^{(i)} S_{1},{ }^{(i)} S_{2}, \ldots,{ }^{(i)} S_{n_{i}}
$$

corresponding to $m$ different levels of detector noise or $m$ different periods of detector operation. (Here $i$ $=1,2, \ldots, m$.) Each of these measurements is taken over an identical time interval of length $T$. These measurements can be thought of as realizations of $m$ random variables ${ }^{(i)} S$, each having the same theoretical mean

$$
{ }^{(i)} \mu:=\left\langle{ }^{(i)} S\right\rangle=: \mu,
$$

but different theoretical variances

$$
{ }^{(i)} \sigma^{2}:=\left\langle{ }^{(i)} S^{2}\right\rangle-\left\langle{ }^{(i)} S\right\rangle^{2} .
$$

The equality of the mean values follows because we assume identical normalization conventions on the optimal filters-e.g., $\mu=\Omega_{\alpha} T$ for a power-law spectrum $\Omega_{\mathrm{gw}}(f)$ $=\Omega_{\alpha} f^{\alpha}$. We also assume that all of the measurements ${ }^{(1)} S_{1},{ }^{(1)} S_{2}, \ldots,{ }^{(m)} S_{n_{m}}$ are statistically independent of one another. ${ }^{34}$

For each set of measurements, we can construct the sample mean (or estimator)

$$
\text { (i) } \hat{\mu}:=\frac{1}{n_{i}} \sum_{j=1}^{n_{i}}{ }^{(i)} S_{j} \text {. }
$$

Viewed as a random variable in its own right, ${ }^{(i)} \hat{\mu}$ has mean value

$$
\mu_{i}:=\left\langle{ }^{(i)} \hat{\mu}\right\rangle=\mu
$$

and variance

$$
\sigma_{i}^{2}:=\left\langle{ }^{(i)} \hat{\mu}^{2}\right\rangle-\left\langle{ }^{(i)} \hat{\mu}\right\rangle^{2}=\frac{{ }^{(i)} \sigma^{2}}{n_{i}} .
$$

What we want to do now is combine all the measurements ${ }^{(1)} S_{1},{ }^{(1)} S_{2}, \ldots,{ }^{(m)} S_{n_{m}}$ (or, equivalently, combine the sample means $\left.{ }^{(1)} \hat{\mu},{ }^{(2)} \hat{\mu}, \ldots,{ }^{(m)} \hat{\mu}\right)$ so as to maximize the overall signal-to-noise ratio. We thus use a weighted average to define the estimator

\footnotetext{
${ }^{34}$ As mentioned in Sec. IV A, this means that the measurements are taken over distinct, nonoverlapping periods of operation, with $T \gg$ the light travel time $d / c$ between the two detectors.
} 


$$
\hat{\mu}:=\frac{\sum_{i=1}^{m} \lambda_{i}{ }^{(i)} \hat{\mu}}{\sum_{j=1}^{m} \lambda_{j}},
$$

and then choose $\lambda_{i}>0$ to maximize the signal-to-noise ratio of $\hat{\mu}$. From Eq. (5.20), it follows that $\hat{\mu}$ has mean value

$$
\mu_{\hat{\mu}}:=\langle\hat{\mu}\rangle=\mu
$$

(which is independent of the choice of $\lambda_{i}$ ) and variance

$$
\sigma_{\hat{\mu}}^{2}:=\left\langle\hat{\mu}^{2}\right\rangle-\langle\hat{\mu}\rangle^{2}=\frac{\sum_{i=1}^{m} \lambda_{i}^{2} \sigma_{i}^{2}}{\left(\sum_{j=1}^{m} \lambda_{j}\right)^{2}} .
$$

The squared signal-to-noise ratio is thus

$$
\operatorname{SNR}_{\hat{\mu}}^{2}:=\frac{\mu_{\hat{\mu}}^{2}}{\sigma_{\hat{\mu}}^{2}}=\mu^{2} \frac{\left(\sum_{j=1}^{m} \lambda_{j}\right)^{2}}{\sum_{i=1}^{m} \lambda_{i}^{2} \sigma_{i}^{2}} .
$$

To find the $\lambda_{i}$ which maximize $\operatorname{SNR}_{\hat{\mu}}^{2}$ (and hence which tell us how to optimally combine data from periods of quiet and noisy detector operation) is quite easy. This is because Eq. (5.23) can be written as a ratio of inner products, just as we were able to write the squared signal-to-noise ratio (3.72) in Sec. III C as a ratio of inner products. Explicitly,

$$
\operatorname{SNR}_{\hat{\mu}}^{2}=\mu^{2} \frac{\left(\lambda, \sigma^{-2}\right)^{2}}{(\lambda, \lambda)}
$$

where the inner product $(\alpha, \beta)$ is defined by

$$
(\alpha, \beta):=\sum_{i=1}^{m} \alpha_{i}^{*} \beta_{i} \sigma_{i}^{2},
$$

for any pair of (complex) sequences $\alpha_{i}$ and $\beta_{i}$ ( $i$ $=1,2, \ldots, m)$. The inner product $(\alpha, \beta)$ is positive-definite, since $\sigma_{i}^{2}$ is real and positive, and it satisfies all of the properties of the ordinary dot product of vectors in threedimensional Euclidean space. Thus, choosing

$$
\lambda_{i} \propto \sigma_{i}^{-2}
$$

maximizes Eq. (5.24) in the same way that choosing $\vec{A}$ proportional to $\vec{B}$ maximizes the ratio $(\vec{A} \cdot \vec{B})^{2} /(\vec{A} \cdot \vec{A})$. By averaging each set of measurements with the inverse of its associated theoretical variance, we give more weight to signal values that are measured when the detectors are quiet than to signal values that are measured when the detectors are noisy. This weighting maximizes the overall signal-to-noise ratio.

For the optimal choice of weights $\lambda_{i}=\sigma_{i}^{-2}$, the inverse of the variance of the optimal estimator

$$
\hat{\mu}_{\text {optimal }}:=\left.\hat{\mu}\right|_{\lambda_{i}=\sigma_{i}^{-2}}
$$

has a very simple form

$$
\sigma_{\text {optimal }}^{-2}=\sum_{i=1}^{m} \sigma_{i}^{-2}=\sum_{i=1}^{m} n_{i}^{(i)} \sigma^{-2} .
$$

This result says that the variances for the optimal estimator add like electrical resistors in parallel. The squared signal-tonoise ratio of the optimal estimator also has a very simple form

$$
\mathrm{SNR}_{\mathrm{optimal}}^{2}=\sum_{i=1}^{m} \mathrm{SNR}_{i}^{2}=\sum_{i=1}^{m} n_{i}{ }^{(i)} \mathrm{SNR}^{2},
$$

where

$$
\mathrm{SNR}_{i}:=\frac{\mu_{i}}{\sigma_{i}}=\frac{\mu}{\sigma_{i}}
$$

and

$$
{ }^{(i)} \mathrm{SNR}:=\frac{{ }^{(i)} \mu}{{ }^{(i)} \sigma}=\frac{\mu}{{ }^{(i)} \sigma} .
$$

Thus, the squared signal-to-noise ratio of the optimal estimator is simply a sum of the squared signal-to-noise ratios for each ${ }^{(i)} S$ after $n_{i}$ observation periods, each of length $T$.

It is instructive to compare the results for the optimal estimator $\hat{\mu}_{\text {optimal }}$ with those for the "naive" estimator

$$
\hat{\mu}_{\text {naive }}:=\frac{1}{n_{\text {tot }}} \sum_{i=1}^{m} \sum_{j=1}^{n_{i}}{ }^{(i)} S_{j}
$$

which simply averages all of the signal estimates, paying no attention to the different variances ${ }^{(i)} \sigma^{2}$ associated with the ${ }^{(i)} S$. The naive estimator $\hat{\mu}_{\text {naive }}$ corresponds to Eq. (5.20) with $\lambda_{i}=n_{i}$ and $n_{\text {tot }}:=\sum_{i=1}^{m} n_{i}$. One can show that

$$
\sigma_{\text {naive }}^{2}=\frac{1}{n_{\text {tot }}^{2}} \sum_{i=1}^{m} n_{i}^{2} \sigma_{i}^{2}=\frac{1}{n_{\text {tot }}^{2}} \sum_{i=1}^{m} n_{i}^{(i)} \sigma^{2}
$$

and

$$
\mathrm{SNR}_{\text {naive }}^{-2}=\frac{1}{n_{\text {tot }}^{2}} \sum_{i=1}^{m} n_{i}^{2} \mathrm{SNR}_{i}^{-2}=\frac{1}{n_{\text {tot }}^{2}} \sum_{i=1}^{m} n_{i}{ }^{(i)} \mathrm{SNR}^{-2} .
$$

Note that in addition to the factors of $1 / n_{\text {tot }}^{2}$, the signs of the exponents for the variances and signal-to-noise ratios in Eqs. (5.33) and (5.34) are opposite to those in Eqs. (5.28) and (5.29).

To give a numerical example, suppose we have just two different periods of detector operation, with the second period twice as noisy as the first-i.e., ${ }^{(2)} \sigma=2 x^{(1)} \sigma$. Since $\sigma_{i}^{2}={ }^{(i)} \sigma^{2} / n_{i}$, it follows that if there are 4 times as many measurements during the noisy period (i.e., $n_{2}=4 n_{1}$ ), then $\sigma_{1}^{2}=\sigma_{2}^{2}$. Intuition suggests that the optimal way of combining the measurements for this case is to weight the estimators 
${ }^{(1)} \hat{\mu}$ and ${ }^{(2)} \hat{\mu}$ equally (i.e., $\lambda_{1}=\lambda_{2}=\sigma_{1}^{-2}$ ). This agrees with the above mathematical analysis, and yields a squared signal-to-noise ratio for the optimal estimator equal to

$$
\mathrm{SNR}_{\text {optimal }}^{2}=2 n_{1}{ }^{(1)} \mathrm{SNR}^{2}=2 n_{1} \frac{\mu^{2}}{(1) \sigma^{2}} \text {. }
$$

For the naive estimator,

$$
\mathrm{SNR}_{\text {naive }}^{2}=\frac{25}{17} n_{1}{ }^{(1)} \mathrm{SNR}^{2}=\frac{25}{17} n_{1} \frac{\mu^{2}}{(1) \sigma^{2}}
$$

Thus, for this particular example, the signal-to-noise ratio for the optimal estimator is $\sqrt{34 / 25}=1.17$ times larger than that for the naive estimator.

\section{Multiple detector pairs}

Combining measurements from multiple detector pairs in order to increase the sensitivity of a stochastic background search is identical to combining different sets of measurements corresponding to quiet and noisy periods of detector operation in order to maximize the overall signal-to-noise ratio. The only difference between the two is one of interpretation and notation. In Sec. V B,

$$
{ }^{(i)} S_{1},{ }^{(i)} S_{2}, \ldots{ }^{(i)} S_{n_{i}}
$$

denoted $n_{i}$ different measurements, each of length $T$, of the optimally filtered cross-correlation signal ${ }^{(i)} S$ when the level of detector noise was characterized by the variance

$$
{ }^{(i)} \sigma_{i}^{2}:=\left\langle{ }^{(i)} S^{2}\right\rangle-\left\langle{ }^{(i)} S\right\rangle^{2} \text {. }
$$

In this section, for multiple detector pairs,

$$
{ }^{(i j)} S_{1},{ }^{(i j)} S_{2}, \ldots,{ }^{(i j)} S_{n_{i j}}
$$

denote $n_{i j}$ different measurements, again each of length $T$, of the optimally filtered cross-correlation signal ${ }^{(i j)} S$ between the $i$ th and $j$ th detectors. ${ }^{35}$ As usual,

$$
{ }^{(i j)} \sigma_{i}^{2}:=\left\langle{ }^{(i j)} S^{2}\right\rangle-\left\langle{ }^{(i j)} S\right\rangle^{2}
$$

denotes the variance of the cross-correlation signal ${ }^{(i j)} S$.

Provided that each cross-correlation signal measurement occurs over the same time interval $T$ (which should be $\gg$ the light travel time between any pair of detectors), and that the optimal filter functions for each detector pair have identical normalizations [e.g., $\lambda$ in Eq. (3.73) is chosen so that ${ }^{(i j)} \mu$ $=\left\langle{ }^{(i j)} S\right\rangle=\Omega_{\alpha} T$ for a stochastic gravity-wave background

\footnotetext{
${ }^{35}$ In Sec. V B, $i=1,2, \ldots, m$ labeled $m$ different levels of detector noise or $m$ different periods of detector operation. In this section, $i, j=1,2, \ldots, l$ label $l$ different detectors, and $m:=l(l-1) / 2$ is the number of different detector pairs.
}

having a power-law spectrum $\left.\Omega_{\mathrm{gw}}(f)=\Omega_{\alpha} f^{\alpha}\right]$, the mathematical analysis of Sec. V B goes through unchanged. ${ }^{36}$ The optimal way of combining the estimators

$$
{ }^{(i j)} \hat{\mu}:=\frac{1}{n_{i j}} \sum_{k=1}^{n_{i j}}{ }^{(i j)} S_{k}
$$

for each detector pair is

$$
\hat{\mu}:=\frac{\sum_{i=1}^{l} \sum_{j<i}^{l} \lambda_{i j}{ }^{(i j)} \hat{\mu}}{\sum_{i=1}^{l} \sum_{j<i}^{l} \lambda_{i j}},
$$

where $^{37}$

$$
\lambda_{i j}=\sigma_{i j}^{-2}=n_{i j}{ }^{(i j)} \sigma^{-2} .
$$

The inverse of the variance for the optimal estimator

$$
\hat{\mu}_{\text {optimal }}:=\left.\hat{\mu}\right|_{\lambda_{i j}=\sigma_{i j}^{-2}}
$$

is given by

$$
\sigma_{\text {optimal }}^{-2}=\sum_{i=1}^{l} \sum_{j<i}^{l} n_{i j}{ }^{(i j)} \sigma^{-2} .
$$

The squared signal-to-noise ratio for the optimal estimator is given by

$$
\mathrm{SNR}_{\text {optimal }}^{2}=\sum_{i=1}^{l} \sum_{j<i}^{l} n_{i j}{ }^{(i j)} \mathrm{SNR}^{2} .
$$

These equations should be compared with Eqs. (5.28) and (5.29) in Sec. V B.

Using Eq. (5.45) for the inverse variance of the optimal estimator, we can derive an expression for the minimum value of $\Omega_{0}$ after 4 months of observation (i.e., $T_{\text {tot }}$ $=10^{7} \mathrm{sec}$ ), for a false alarm rate equal to $5 \%$ and a detec-

\footnotetext{
${ }^{36}$ Although cross-correlation signals ${ }^{(i j)} S$ and ${ }^{(k l)} S$ taken during the same time interval $T$ are correlated, i.e.,

$$
\operatorname{cov}\left\{{ }^{(i j)} S,{ }^{(k l)} S\right\}:=\left\langle{ }^{(i j)} S{ }^{(k l)} S\right\rangle-\left\langle\langle { } ^ { ( i j ) } S \rangle \left\langle\left\langle{ }^{(k l)} S\right\rangle \neq 0,\right.\right.
$$

the variance-covariance matrix $C_{(i j)(k l)}:=\operatorname{cov}\left\{{ }^{(i j)} S,{ }^{(k l)} S\right\}$ is dominated by the diagonal terms $C_{(i j)(i j)}=\operatorname{cov}\left\{{ }^{(i j)} S,{ }^{(i j)} S\right\}$ $=:^{(i j)} \sigma^{2}$ in the large noise approximation. Thus, in practice, one can treat all of the measurements ${ }^{(12)} S_{1},{ }^{(12)} S_{2}, \ldots,{ }^{(l-1, l)} S_{n_{l-1, l}}$, as effectively uncorrelated. This is because the detector noises (which are statistically independent of one another) are the only contributors to ${ }^{(i j)} \sigma^{2}$ in this approximation.

${ }^{37}$ For completeness, we note that the optimal way of combining correlated random variables $x_{1}, \ldots, x_{m}$ is given by $\hat{\mu}$ $:=\sum_{i=1}^{m} \lambda_{i} x_{i} / \sum_{j=1}^{m} \lambda_{j}$, where $\lambda_{i}=\sum_{j=1}^{m}\left(C^{-1}\right)_{i j}$ and $\left(C^{-1}\right)_{i j}$ is the inverse of the variance-covariance matrix $C_{i j}:=\operatorname{cov}\left\{x_{i}, x_{j}\right\}$. When the variance-covariance matrix is dominated by the diagonal terms $C_{i i}=\operatorname{cov}\left\{x_{i}, x_{i}\right\}=: \sigma_{i}^{2}$, the optimal combination of data reduces (approximately) to the uncorrelated result $\lambda_{i}=\sigma_{i}^{-2}$. As argued in the previous footnote, this is what happens for the cross-correlation signals $x_{i} \leftrightarrow^{(i j)} S$.
} 
tion rate equal to $95 \%$, where we optimally combine data from different detector pairs. In order to simplify the analysis, we will assume that $T_{\text {tot }}=n T$, where $n:=n_{i j}$ is the same for all pairs of detectors.

For a single pair of detectors, the minimum value of $\Omega_{0}$ is given by

$$
{ }^{(i j)} \Omega_{0}^{95 \%, 5 \%}=\frac{2 \times 1.65}{10^{7} \sec } \sqrt{n}{ }^{(i j)} \sigma,
$$

where

$$
{ }^{(i j)} \sigma^{2} \approx T\left(\frac{10 \pi^{2}}{3 H_{0}^{2}}\right)^{2}\left[\int_{-\infty}^{\infty} d f \frac{\gamma^{2}(|f|)}{f^{6} P_{i}(|f|) P_{j}(|f|)}\right]^{-1} .
$$

The above expression for ${ }^{(i j)} \sigma^{2}$ follows from Eqs. (3.68) and (3.73), together with the normalization condition $\mu=\Omega_{0} T$ for a stochastic background having a constant frequency spectrum $\Omega_{\mathrm{gw}}(f)=\Omega_{0}$. The factor of $2 \times 1.65$ in Eq. (5.47) comes from the choice of the false alarm and detection rates. [See, e.g., Eq. (4.37).] Table II in Sec. VI D contains values of ${ }^{(i j)} \Omega_{0}^{95 \%, 5 \%} h_{100}^{2}$ for different detector pairs.

For the optimal combination of data from multiple detector pairs, we have

$$
\left(\left.\Omega_{0}^{95 \%, 5 \%}\right|_{\text {optimal }}\right)^{-2}=\left(\frac{2 \times 1.65}{10^{7} \mathrm{sec}}\right)^{-2} n^{-1} \sum_{i=1}^{l} \sum_{j<i}^{l}{ }^{(i j)} \sigma^{-2} .
$$

This can also be written as

$$
\left.\left(\left.\Omega_{0}^{95 \%, 5 \%}\right|_{\text {optimal }}\right)^{-2}=\sum_{i=1}^{l} \sum_{j<i}^{l}{ }^{\left({ }^{(i j)}\right)} \Omega_{0}^{95 \%, 5 \%}\right)^{-2} .
$$

Tables III and IV in Sec. VID contain values of $\left.\Omega_{0}^{95 \%, 5 \%}\right|_{\text {optimal }}$ for the optimal combination of data from multiple detector pairs, for all possible triples and quadruples of the five major interferometers.

\section{Four-detector correlation}

Rather than combine data from multiple detector pairs as described in the previous section, one can directly correlate the outputs of 4 detectors (or, in general, $2 N$ detectors) in a manner analogous to the single 2-detector correlation described in Sec. III C. ${ }^{38}$ In fact, it turns out that one can write down expressions for the optimally filtered squared signalto-noise ratio and the inverse of the square of $\Omega_{0}^{95 \%, 5 \%}$ for the 4-detector correlation in terms of a simple sum of products of the corresponding quantities for the individual detector pairs. As noted above, the analysis given here can easily be generalized to the case of $2 N$ detectors. At the end of the section, we summarize our results by writing down the key equations in the general $2 N$-detector form.

To begin, we define the 4-detector correlation signal $S$ to be the integrated product

$$
S:=\int_{-T / 2}^{T / 2} d t_{1} \cdots \int_{-T / 2}^{T / 2} d t_{4} s_{1}\left(t_{1}\right) \cdots s_{4}\left(t_{4}\right) Q\left(t_{1}, \ldots, t_{4}\right),
$$

where

$$
s_{i}(t):=h_{i}(t)+n_{i}(t)
$$

are the outputs of the detectors $(i=1, \ldots, 4)$, and $Q\left(t_{1}, \ldots, t_{4}\right)$ is an arbitrary filter function, which we will determine shortly. To save some writing in what follows, we will use the shorthand notation

$$
\int_{-T / 2}^{T / 2} d^{4} t \equiv \int_{-T / 2}^{T / 2} d t_{1} \ldots \int_{-T / 2}^{T / 2} d t_{4}
$$

Throughout, we will assume that the gravitational strains $h_{i}(t)$ satisfy the statistical properties listed in Sec. II B. We will also assume that the noises $n_{i}(t)$ intrinsic to the detectors are (i) stationary, (ii) Gaussian, (iii) statistically independent of one another and of the gravitational strains, and (iv) much larger in magnitude than the gravitational strains. The goal is to determine the filter function $Q\left(t_{1}, \ldots, t_{4}\right)$ that maximizes the signal-to-noise ratio $\mathrm{SNR}:=\mu / \sigma$ of $S$.

Let us start by calculating the variance $\sigma^{2}$. Since we are assuming that the noises intrinsic to the detectors are statistically independent of one another and of the gravitational strains, and that they are much larger in magnitude than the gravitational strains, it follows that

$$
\begin{aligned}
\sigma^{2}: & =\left\langle S^{2}\right\rangle-\langle S\rangle^{2} \approx\left\langle S^{2}\right\rangle \\
& =\int_{-T / 2}^{T / 2} d^{4} t \int_{-T / 2}^{T / 2} d^{4} t^{\prime}\left\langle s_{1}\left(t_{1}\right) \cdots s_{4}\left(t_{4}\right) s_{1}\left(t_{1}^{\prime}\right) \cdots s_{4}\left(t_{4}^{\prime}\right)\right\rangle Q\left(t_{1}, \ldots, t_{4}\right) Q\left(t_{1}^{\prime}, \ldots, t_{4}^{\prime}\right) \\
& \approx \int_{-T / 2}^{T / 2} d^{4} t \int_{-T / 2}^{T / 2} d^{4} t^{\prime}\left\langle n_{1}\left(t_{1}\right) \cdots n_{4}\left(t_{4}\right) n_{1}\left(t_{1}^{\prime}\right) \cdots n_{4}\left(t_{4}^{\prime}\right)\right\rangle Q\left(t_{1}, \ldots, t_{4}\right) Q\left(t_{1}^{\prime}, \ldots, t_{4}^{\prime}\right) \\
& =\int_{-T / 2}^{T / 2} d^{4} t \int_{-T / 2}^{T / 2} d^{4} t^{\prime}\left\langle n_{1}\left(t_{1}\right) n_{1}\left(t_{1}^{\prime}\right)\right\rangle \cdots\left\langle n_{4}\left(t_{4}\right) n_{4}\left(t_{4}^{\prime}\right)\right\rangle Q\left(t_{1}, \ldots, t_{4}\right) Q\left(t_{1}^{\prime}, \ldots, t_{4}^{\prime}\right) .
\end{aligned}
$$

[See Eqs. (3.19)-(3.22).] Using the definition (3.23) of the noise power spectra $P_{i}(|f|)$, Eq. (5.57) can be rewritten as

\footnotetext{
${ }^{38}$ The correlation of 3 detectors (or, in general, $2 N+1$ detectors) yields a signal that has zero mean.
} 


$$
\begin{aligned}
\sigma^{2} \approx & \left(\frac{1}{2}\right)^{4} \int_{-\infty}^{\infty} d^{4} f P_{1}\left(\left|f_{1}\right|\right) \cdots P_{4}\left(\left|f_{4}\right|\right) \int_{-T / 2}^{T / 2} d^{4} t e^{i 2 \pi f_{1} t_{1}} \cdots e^{i 2 \pi f_{4} t_{4}} Q\left(t_{1}, \ldots, t_{4}\right) \\
& \times \int_{-T / 2}^{T / 2} d^{4} t^{\prime} e^{-i 2 \pi f_{1} t_{1}^{\prime}} \ldots e^{-i 2 \pi f_{4} t_{4}^{\prime}} Q\left(t_{1}^{\prime}, \ldots, t_{4}^{\prime}\right)
\end{aligned}
$$

where

$$
\int_{-\infty}^{\infty} d^{4} f \equiv \int_{-\infty}^{\infty} d f_{1} \cdots \int_{-\infty}^{\infty} d f_{4} .
$$

If we further define the (finite-time) Fourier transform

$$
\widetilde{Q}\left(f_{1}, \ldots, f_{4}\right):=\int_{-T / 2}^{T / 2} d^{4} t e^{-i 2 \pi f_{1} t_{1}} \ldots e^{-i 2 \pi f_{4} t_{4}} Q\left(t_{1}, \ldots, t_{4}\right),
$$

which has as its inverse (for $-T / 2<t_{1}, \ldots, t_{4}<T / 2$ )

$$
Q\left(t_{1}, \ldots, t_{4}\right)=\int_{-\infty}^{\infty} d^{4} f e^{i 2 \pi f_{1} t_{1}} \ldots e^{i 2 \pi f_{4} t_{4}} \widetilde{Q}\left(f_{1}, \ldots, f_{4}\right),
$$

we obtain

$$
\sigma^{2} \approx\left(\frac{1}{2}\right)^{4} \int_{-\infty}^{\infty} d^{4} f P_{1}\left(\left|f_{1}\right|\right) \cdots P_{4}\left(\left|f_{4}\right|\right)\left|\widetilde{Q}\left(f_{1}, \ldots, f_{4}\right)\right|^{2} .
$$

This can be written in an even more convenient form if we define an inner product

$$
(A, B):=\int_{-\infty}^{\infty} d^{4} f P_{1}\left(\left|f_{1}\right|\right) \cdots P_{4}\left(\left|f_{4}\right|\right) A^{*}\left(f_{1}, \ldots, f_{4}\right) B\left(f_{1}, \ldots, f_{4}\right),
$$

where $A\left(f_{1}, \ldots, f_{4}\right)$ and $B\left(f_{1}, \ldots, f_{4}\right)$ are any two complex-valued functions of four variables. [See Eq. (3.69).] Note that the inner product is positive-definite since $P_{i}(|f|)$ $>0$. Using Eq. (5.63) it follows that

$$
\sigma^{2} \approx\left(\frac{1}{2}\right)^{4}(\widetilde{Q}, \widetilde{Q})
$$

To calculate the mean value $\mu$, we proceed in a similar manner. Since the noises intrinsic to the detectors are statistically independent of one another and of the gravitational strains, it follows that

$$
\begin{aligned}
\mu & :=\langle S\rangle \\
& =\int_{-T / 2}^{T / 2} d^{4} t\left\langle s_{1}\left(t_{1}\right) \cdots s_{4}\left(t_{4}\right)\right\rangle Q\left(t_{1}, \ldots, t_{4}\right) \\
& =\int_{-T / 2}^{T / 2} d^{4} t\left\langle h_{1}\left(t_{1}\right) \cdots h_{4}\left(t_{4}\right)\right\rangle Q\left(t_{1}, \ldots, t_{4}\right) .
\end{aligned}
$$

This can be expanded to

$$
\begin{aligned}
\mu= & \int_{-T / 2}^{T / 2} d^{4} t\left(\left\langle h_{1}\left(t_{1}\right) h_{2}\left(t_{2}\right)\right\rangle\left\langle h_{3}\left(t_{3}\right) h_{4}\left(t_{4}\right)\right\rangle\right. \\
& +\langle 13\rangle\langle 24\rangle+\langle 14\rangle\langle 23\rangle) Q\left(t_{1}, \ldots, t_{4}\right)
\end{aligned}
$$

by using the "factorization", property

$$
\left\langle x_{1} x_{2} x_{3} x_{4}\right\rangle=\left\langle x_{1} x_{2}\right\rangle\left\langle x_{3} x_{4}\right\rangle+\left\langle x_{1} x_{3}\right\rangle\left\langle x_{2} x_{4}\right\rangle+\left\langle x_{1} x_{4}\right\rangle\left\langle x_{2} x_{3}\right\rangle
$$

for Gaussian random variables $x_{1}, x_{2}, x_{3}, x_{4}$ each having zero mean. $^{39}$

To express $\mu$ in terms of the inner product (5.63), we first use Eq. (5.61) to rewrite $Q\left(t_{1}, \ldots, t_{4}\right)$ in terms of its Fourier transform $\widetilde{Q}\left(f_{1}, \ldots, f_{4}\right)$ :

$$
\begin{aligned}
\mu= & \int_{-T / 2}^{T / 2} d^{4} t\left(\left\langle h_{1}\left(t_{1}\right) h_{2}\left(t_{2}\right)\right\rangle\left\langle h_{3}\left(t_{3}\right) h_{4}\left(t_{4}\right)\right\rangle\right. \\
& +\langle 13\rangle\langle 24\rangle+\langle 14\rangle\langle 23\rangle) \\
& \times \int_{-\infty}^{\infty} d^{4} f e^{i 2 \pi f_{1} t_{1}} \ldots e^{i 2 \pi f_{4} t_{4}} \widetilde{Q}\left(f_{1}, \ldots, f_{4}\right) .
\end{aligned}
$$

Then by interchanging the order of integrations and rearranging terms in the integrand, we see that

$$
\begin{aligned}
\mu= & \int_{-\infty}^{\infty} d^{4} f \int_{-T / 2}^{T / 2} d^{4} t e^{i 2 \pi f_{1} t_{1}} \ldots e^{i 2 \pi f_{4} t_{4}}\left(\left\langle h_{1}\left(t_{1}\right) h_{2}\left(t_{2}\right)\right\rangle\right. \\
& \left.\times\left\langle h_{3}\left(t_{3}\right) h_{4}\left(t_{4}\right)\right\rangle+\langle 13\rangle\langle 24\rangle+\langle 14\rangle\langle 23\rangle\right) \\
& \times \widetilde{Q}\left(f_{1}, \ldots, f_{4}\right)
\end{aligned}
$$

\footnotetext{
${ }^{39}$ In Eq. (5.68), $\langle 13\rangle\langle 24\rangle$ is used as a shorthand notation for $\left\langle h_{1}\left(t_{1}\right) h_{3}\left(t_{3}\right)\right\rangle\left\langle h_{2}\left(t_{2}\right) h_{4}\left(t_{4}\right)\right\rangle$, etc.
} 
or, equivalently,

$$
\mu=(A, \widetilde{Q}),
$$

where

$$
\begin{aligned}
A\left(f_{1}, \ldots, f_{4}\right):= & \frac{1}{P_{1}\left(\left|f_{1}\right|\right) \cdots P_{4}\left(\left|f_{4}\right|\right)} \int_{-T / 2}^{T / 2} d^{4} t \\
& \times e^{-i 2 \pi f_{1} t_{1}} \cdots e^{-i 2 \pi f_{4} t_{4}}\left(\left\langle h_{1}\left(t_{1}\right) h_{2}\left(t_{2}\right)\right\rangle\right. \\
& \left.\times\left\langle h_{3}\left(t_{3}\right) h_{4}\left(t_{4}\right)\right\rangle+\langle 13\rangle\langle 24\rangle+\langle 14\rangle\langle 23\rangle\right) .
\end{aligned}
$$

To simplify this expression for $A\left(f_{1}, \ldots, f_{4}\right)$, we expand the expectation values $\left\langle h_{i}\left(t_{i}\right) h_{j}\left(t_{j}\right)\right\rangle$ as

$$
\left\langle h_{i}\left(t_{i}\right) h_{j}\left(t_{j}\right)\right\rangle=\int_{-\infty}^{\infty} d f e^{i 2 \pi f\left(t_{i}-t_{j}\right)} H_{i j}(f),
$$

where

$$
H_{i j}(f)=\frac{3 H_{0}^{2}}{20 \pi^{2}}|f|^{-3} \Omega_{\mathrm{gw}}(|f|) \gamma_{i j}(|f|),
$$

and $\gamma_{i j}(f)$ denotes the overlap reduction function between the $i j$ detector pair. [See Eqs. (3.58) and (3.59).] Substituting Eq. (5.74) into (5.73) yields

$$
\begin{aligned}
A\left(f_{1}, \ldots, f_{4}\right):= & \frac{1}{P_{1}\left(\left|f_{1}\right|\right) \cdots P_{4}\left(\left|f_{4}\right|\right)} \\
& \times \int_{-\infty}^{\infty} d f \int_{-\infty}^{\infty} d f^{\prime} H_{12}(f) H_{34}\left(f^{\prime}\right) \\
& \times \int_{-T / 2}^{T / 2} d^{4} t e^{-i 2 \pi f_{1} t_{1}} \cdots e^{-i 2 \pi f_{4} t_{4}} \\
& \times e^{i 2 \pi f\left(t_{1}-t_{2}\right)} e^{i 2 \pi f^{\prime}\left(t_{3}-t_{4}\right)}+13,24+14,23,
\end{aligned}
$$

where 13,24 and 14,23 denote the analogous terms with the appropriate interchange of detector indices $1, \ldots, 4$. Since

$$
\delta_{T}(f)=\int_{-T / 2}^{T / 2} d t e^{i 2 \pi f t}
$$

[see Eq. 3.27], we can explicitly integrate over the time variables $t_{1}, \ldots, t_{4}$ :

$$
\begin{aligned}
A\left(f_{1}, \ldots, f_{4}\right):= & \frac{1}{P_{1}\left(\left|f_{1}\right|\right) \cdots P_{4}\left(\left|f_{4}\right|\right)} \\
& \times \int_{-\infty}^{\infty} d f \int_{-\infty}^{\infty} d f^{\prime} H_{12}(f) H_{34}\left(f^{\prime}\right) \\
& \times \delta_{T}\left(f_{1}-f\right) \delta_{T}\left(f_{2}+f\right) \delta_{T}\left(f_{3}-f^{\prime}\right) \\
& \times \delta_{T}\left(f_{4}+f^{\prime}\right)+13,24+14,23 .
\end{aligned}
$$

Also, since the noise power spectra $P_{i}\left(\left|f_{i}\right|\right)$ and filter function $\widetilde{Q}\left(f_{1}, \ldots, f_{4}\right)$ are not expected to vary much over the support, $1 / T$, of $\delta_{T}\left(f_{1}-f\right)$ and $\delta_{T}\left(f_{3}-f^{\prime}\right)$, we are justified in approximating these two finite-time delta functions by ordinary Dirac delta functions $\delta\left(f_{1}-f\right)$ and $\delta\left(f_{3}-f^{\prime}\right)$. This approximation allows us to eliminate the integrations over $f$ and $f^{\prime}$, yielding

$$
\begin{aligned}
A\left(f_{1}, \ldots, f_{4}\right) \approx & \frac{H_{12}\left(f_{1}\right)}{P_{1}\left(\left|f_{1}\right|\right) P_{2}\left(\left|f_{2}\right|\right)} \delta_{T}\left(f_{1}+f_{2}\right) \\
& \times \frac{H_{34}\left(f_{3}\right)}{P_{3}\left(\left|f_{3}\right|\right) P_{4}\left(\left|f_{4}\right|\right)} \delta_{T}\left(f_{3}+f_{4}\right) \\
& +13,24+14,23 .
\end{aligned}
$$

Given the above expressions for $\sigma^{2}, \mu$, and $A\left(f_{1}, \ldots, f_{4}\right)$, it is now a simple matter to evaluate the squared signal-to-noise ratio of the 4-detector correlation $S$, and to determine the filter function $\widetilde{Q}\left(f_{1}, \ldots, f_{4}\right)$ that maximizes this ratio. In terms of the inner product (5.63), we have

$$
\mathrm{SNR}^{2}:=\frac{\mu^{2}}{\sigma^{2}} \approx 2^{4} \frac{(A, \widetilde{Q})^{2}}{(\widetilde{Q}, \widetilde{Q})}
$$

As we saw already in Secs. III C and V A, such a ratio of inner products is maximized by choosing

$$
\widetilde{Q}\left(f_{1}, \ldots, f_{4}\right)=\lambda A\left(f_{1}, \ldots, f_{4}\right),
$$

where $\lambda$ is an arbitrary (real) overall normalization constant. For this choice of $\widetilde{Q}\left(f_{1}, \ldots, f_{4}\right)$, the value of the squared signal-to-noise ratio is given by

$$
\mathrm{SNR}_{\text {optimal }}^{2} \approx 2^{4}(A, A) \text {. }
$$

To find an explicit expression for $\mathrm{SNR}_{\text {optimal }}^{2}$, we substitute Eq. (5.79) into the RHS of Eq. (5.82) and expand the product of $A\left(f_{1}, \ldots, f_{4}\right)$ with itself. This leads to nine different terms: three diagonal (i.e., squared) terms and six offdiagonal terms. The diagonal terms are given by

$$
\begin{aligned}
\text { diagonal terms }= & 2^{4} \int_{-\infty}^{\infty} d^{4} f \frac{H_{12}^{2}\left(f_{1}\right)}{P_{1}\left(\left|f_{1}\right|\right) P_{2}\left(\left|f_{2}\right|\right)} \\
& \times \delta_{T}^{2}\left(f_{1}+f_{2}\right) \frac{H_{34}^{2}\left(f_{3}\right)}{P_{3}\left(\left|f_{3}\right|\right) P_{4}\left(\left|f_{4}\right|\right)} \\
& \times \delta_{T}^{2}\left(f_{3}+f_{4}\right)+13,24+14,23 .
\end{aligned}
$$

A typical off-diagonal term is given by

$$
\begin{aligned}
\text { off-diagonal term }= & 2^{4} \int_{-\infty}^{\infty} d^{4} f \frac{1}{P_{1}\left(\left|f_{1}\right|\right) \cdots P_{4}\left(\left|f_{4}\right|\right)} \\
& \times H_{12}\left(f_{1}\right) H_{34}\left(f_{3}\right) H_{13}\left(f_{1}\right) H_{24}\left(f_{2}\right) \\
& \times \delta_{T}\left(f_{1}+f_{2}\right) \delta_{T}\left(f_{3}+f_{4}\right) \\
& \times \delta_{T}\left(f_{1}+f_{3}\right) \delta_{T}\left(f_{2}+f_{4}\right)
\end{aligned}
$$

Let us evaluate each of these terms separately. First, for the diagonal terms, by approximating one of the two finitetime delta functions in each of the factors $\delta_{T}^{2}\left(f_{1}+f_{2}\right)$ and 
$\delta_{T}^{2}\left(f_{3}+f_{3}\right)$ in Eq. (5.83) by ordinary Dirac delta functions, and by evaluating the others at $f_{1}+f_{2}=0$ and $f_{3}+f_{4}=0$, we eliminate two of the integrations, and introduce a factor of $T^{2}$ :

$$
\begin{aligned}
\text { diagonal terms } \approx & 2{ }^{4} T^{2} \int_{-\infty}^{\infty} d f \frac{H_{12}^{2}(f)}{P_{1}(|f|) P_{2}(|f|)} \int_{-\infty}^{\infty} d f^{\prime} \frac{H_{34}^{2}\left(f^{\prime}\right)}{P_{3}\left(\left|f^{\prime}\right|\right) P_{4}\left(\left|f^{\prime}\right|\right)}+13,24+14,23 \\
= & \left(\frac{3 H_{0}^{2}}{10 \pi^{2}}\right)^{4} T^{2} \int_{-\infty}^{\infty} d f \frac{\gamma_{12}^{2}(|f|) \Omega_{\mathrm{gw}}^{2}(|f|)}{f^{6} P_{1}(|f|) P_{2}(|f|)} \int_{-\infty}^{\infty} d f^{\prime} \frac{\gamma_{34}^{2}\left(\left|f^{\prime}\right|\right) \Omega_{\mathrm{gw}}^{2}\left(\left|f^{\prime}\right|\right)}{f^{\prime 6} P_{3}\left(\left|f^{\prime}\right|\right) P_{4}\left(\left|f^{\prime}\right|\right)} \\
& +13,24+14,23 \\
= & { }^{(12)} \mathrm{SNR}^{2}{ }^{\left({ }^{34}\right)} \mathrm{SNR}^{2}+{ }^{(13)} \mathrm{SNR}^{2}{ }^{(24)} \mathrm{SNR}^{2}+{ }^{(14)} \mathrm{SNR}^{2}{ }^{(23)} \mathrm{SNR}^{2},
\end{aligned}
$$

where ${ }^{(i j)} \mathrm{SNR}^{2}$ denotes the squared signal-to-noise ratios for the optimally filtered cross-correlation signal for the $i j$ detector pair, which we derived in Sec. III C.

For the off-diagonal term (5.84), we can again approximate $\delta_{T}\left(f_{1}+f_{2}\right)$ and $\delta_{T}\left(f_{3}+f_{4}\right)$ by ordinary Dirac delta functions to obtain

$$
\begin{aligned}
\text { off-diagonal term } \approx & 2^{4} \int_{-\infty}^{\infty} d f_{1} \int_{-\infty}^{\infty} d f_{3} \frac{1}{P_{1}\left(\left|f_{1}\right|\right) P_{2}\left(\left|f_{1}\right|\right) P_{3}\left(\left|f_{3}\right|\right) P_{4}\left(\left|f_{3}\right|\right)} \\
& \times H_{12}\left(f_{1}\right) H_{34}\left(f_{3}\right) H_{13}\left(f_{1}\right) H_{24}\left(-f_{1}\right) \delta_{T}^{2}\left(f_{1}+f_{3}\right) .
\end{aligned}
$$

By further approximating one of the finite-time delta functions in $\delta_{T}^{2}\left(f_{1}+f_{3}\right)$ by an ordinary Dirac delta function, and by evaluating the other at $f_{1}+f_{3}=0$, we eliminate one more integration and introduce a factor of $T$ :

off-diagonal term

$$
\approx 2^{4} T \int_{-\infty}^{\infty} d f \frac{H_{12}(f) H_{34}(f) H_{13}(f) H_{24}(f)}{P_{1}(|f|) P_{2}(|f|) P_{3}(|f|) P_{4}(|f|)} .
$$

But since this term grows like $T$, while the diagonal terms grow like $T^{2}$, we can (for large observation times) ignore the contribution of the off-diagonal terms to the optimal signalto-noise ratio. The final result is thus ${ }^{40}$

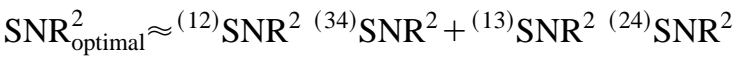

$$
\begin{aligned}
& +{ }^{(14)} \mathrm{SNR}^{2}{ }^{23} \mathrm{SNR}^{2} \text {. }
\end{aligned}
$$

Note that the optimal signal-to-noise ratio for the 4-detector correlation is quadratic in the signal-to-noise ratios for the individual $i j$ detector pairs. This is because the 4-detector correlation signal $S$, given by Eq. (5.51), is quartic in the

\footnotetext{
${ }^{40}$ This expression should be compared with

$$
\mathrm{SNR}_{\text {optimal }}^{2}={ }^{(12)} \mathrm{SNR}^{2}+{ }^{(13)} \mathrm{SNR}^{2}+\cdots+{ }^{(34)} \mathrm{SNR}^{2},
$$
}

which is the squared signal-to-noise ratio that we found in the previous section for the optimal combination of data from multiple detector pairs. [See Eq. (5.46).] detector outputs $s_{i}\left(t_{i}\right)$, while the cross-correlation signal values ${ }^{(i j)} S$ are quadratic in the detector outputs. [See, e.g., Eq. (3.45).]

Given Eq. (5.91), we can now ask the question, what is the minimum value of $\Omega_{0}$ required to detect a stochastic gravity-wave signal $95 \%$ of the time, from data obtained via a 4-detector correlation experiment? Since

$$
\begin{aligned}
S & :=\int_{-T / 2}^{T / 2} d^{4} t s_{1}\left(t_{1}\right) \cdots s_{4}\left(t_{4}\right) Q\left(t_{1}, \ldots, t_{4}\right) \\
& =\int_{-\infty}^{\infty} d^{4} f \tilde{s}_{1}\left(f_{1}\right) \cdots \tilde{s}_{4}\left(f_{4}\right) \widetilde{Q}^{*}\left(f_{1}, \ldots, f_{4}\right)
\end{aligned}
$$

is, effectively, a sum (over $f_{1}, \ldots, f_{4}$ ) of a large number of statistically independent random variables [products of the Fourier amplitudes $\tilde{s}_{i}\left(f_{i}\right)$, which are correlated only when $\left.\left|f_{i}-f_{j}\right|<1 / T\right]$, the central limit theorem guarantees that $S$ will be well-approximated by a Gaussian random variable. Thus, the statistical analysis of Sec. IV is valid for the 4-detector correlation as well. In particular, for a false alarm rate $\alpha=0.05$ and for a detection rate $\gamma=0.95$, the minimum value of $\Omega_{0}$ for the 4-detector correlation is determined by setting the signal-to-noise ratio after $n$ observation periods equal to $2 \times 1.65$ [see Eq. (4.36)]. For a stochastic background having a constant frequency spectrum $\Omega_{\mathrm{gw}}(f)=\Omega_{0}$ and for a total observation time $T_{\text {tot }}:=n T$, the squared signal-to-noise ratio for the optimally filtered 4-detector correlation signal $S$ can be written as 


$$
\begin{aligned}
\operatorname{SNR}_{\text {optimal }}^{2} \approx & \Omega_{0}^{4}\left(\frac{3 H_{0}^{2}}{10 \pi^{2}}\right)^{4} T_{\text {tot }}^{2} \int_{-\infty}^{\infty} d f \frac{\gamma_{12}^{2}(|f|)}{f^{6} P_{1}(|f|) P_{2}(|f|)} \\
& \times \int_{-\infty}^{\infty} d f^{\prime} \frac{\gamma_{34}^{2}\left(\left|f^{\prime}\right|\right)}{f^{\prime 6} P_{3}\left(\left|f^{\prime}\right|\right) P_{4}\left(\left|f^{\prime}\right|\right)} \\
& +13,24+14,23 .
\end{aligned}
$$

Setting $\mathrm{SNR}_{\text {optimal }}=2 \times 1.65$ and rearranging terms yields

$$
\begin{aligned}
& \left(\left.\Omega_{0}^{95 \%, 5 \%}\right|_{\text {optimal }}\right)^{-4}(2 \times 1.65)^{2} \\
& \approx\left(\frac{3 H_{0}^{2}}{10 \pi^{2}}\right)^{4} T_{\text {tot }}^{2} \int_{-\infty}^{\infty} d f \frac{\gamma_{12}^{2}(|f|)}{f^{6} P_{1}(|f|) P_{2}(|f|)} \\
& \quad \times \int_{-\infty}^{\infty} d f^{\prime} \frac{\gamma_{34}^{2}\left(\left|f^{\prime}\right|\right)}{f^{\prime 6} P_{3}\left(\left|f^{\prime}\right|\right) P_{4}\left(\left|f^{\prime}\right|\right)}+13,24+14,23,
\end{aligned}
$$

which can also be written in terms of the minimum values ${ }^{(i j)} \Omega_{0}^{95 \%, 5 \%}$ for the individual $i j$ detector pairs:

$$
\begin{aligned}
& \left(\left.\Omega_{0}^{95 \%, 5 \%}\right|_{\text {optimal }}\right)^{-2} \\
& \approx 2 \times 1.65\left[\left({ }^{(12)} \Omega_{0}^{95 \%, 5 \%}\right)^{-2}\left({ }^{(34)} \Omega_{0}^{95 \%, 5 \%}\right)^{-2}\right. \\
& \quad+13,24+14,23]^{1 / 2} .
\end{aligned}
$$

How does this minimum value of $\Omega_{0}$ compare with those found in previous sections for a single 2-detector correlation and for the optimal combination of data from multiple detector pairs? First, from Eq. (5.95) we see immediately that

$$
\left.\Omega_{0}^{95 \%, 5 \%}\right|_{\text {optimal }} \sim T_{\text {tot }}^{-1 / 2} .
$$

This dependence on the total observation time $T_{\text {tot }}$ is the same as that for ${ }^{(i j)} \Omega_{0}^{95 \%, 5 \%}$ for a single detector pair and for the optimal combination of data from multiple detector pairs:

$$
\begin{aligned}
\left(\left.\Omega_{0}^{95 \%, 5 \%}\right|_{\text {optimal }}\right)^{-2}= & \left({ }^{(12)} \Omega_{0}^{95 \%, 5 \%}\right)^{-2}+\left({ }^{(13)} \Omega_{0}^{95 \%, 5 \%}\right)^{-2} \\
& +\cdots+\left({ }^{(34)} \Omega_{0}^{95 \%, 5 \%}\right)^{-2} .
\end{aligned}
$$

[See Eq. (5.50).] Thus, by correlating 2, 4 (or even $2 N$ ) detectors, one does not change the general dependence of the minimum value of $\Omega_{0}$ on the total observation time $T_{\text {tot }}$. However, the numerical factors multiplying $T_{\text {tot }}^{-1 / 2}$ differ from one another. In fact, it is fairly easy to show that $\left.\Omega_{0}^{95 \%, 5 \%}\right|_{\text {optimal }}$ for the single 4-detector correlation is always greater than that for the optimal combination of data from multiple detector pairs. Thus, in theory, it is better to optimally combine data from multiple detector pairs than to optimally filter a single 4-detector correlation. Table V in Sec. VI D lists values of $\left.\Omega_{0}^{95 \%, 5 \%}\right|_{\text {optimal }} h_{100}^{2}$ for the 4-detector correlations taken from the five major interferometers: LIGOWA, LIGO-LA, VIRGO, GEO-600, and TAMA-300.

Finally, to conclude this section, we rewrite the key equations derived above for the general case of $2 N$ detectors:

(i) The $2 N$-detector correlation signal $S$ is defined by

$$
S:=\int_{-T / 2}^{T / 2} d^{2 N} t s_{1}\left(t_{1}\right) \cdots s_{2 N}\left(t_{2 N}\right) Q\left(t_{1}, \ldots, t_{2 N}\right) .
$$

(ii) The "factorization" property for $2 N$ Gaussian random variables each having zero mean is ${ }^{41}$

$$
\begin{aligned}
\left\langle x_{1} x_{2} \cdots x_{2 N}\right\rangle= & \left\langle x_{1} x_{2}\right\rangle\left\langle x_{3} x_{4}\right\rangle \cdots\left\langle x_{2 N-1} x_{2 N}\right\rangle \\
& + \text { all possible permutations. }
\end{aligned}
$$

(iii) The inner product in terms of which

$$
\mu=(A, \widetilde{Q}) \quad \text { and } \quad \sigma^{2}=\left(\frac{1}{2}\right)^{2 N}(\widetilde{Q}, \widetilde{Q})
$$

is given by

$$
\begin{aligned}
(A, B):= & \int_{-\infty}^{\infty} d^{2 N} f P_{1}\left(\left|f_{1}\right|\right) \cdots P_{2 N}\left(\left|f_{2 N}\right|\right) \\
& \times A^{*}\left(f_{1}, \ldots, f_{2 N}\right) B\left(f_{1}, \ldots, f_{2 N}\right),
\end{aligned}
$$

where $A\left(f_{1}, \ldots, f_{2 N}\right)$ and $B\left(f_{1}, \ldots, f_{2 N}\right)$ are any two complex-valued functions of $2 N$ variables.

(iv) The optimal filter function $Q\left(t_{1}, \ldots, t_{2 N}\right)$ is given in the frequency domain by

$$
\widetilde{Q}\left(f_{1}, \cdots, f_{2 N}\right)=\lambda A\left(f_{1}, \ldots, f_{2 N}\right),
$$

where

$$
\begin{aligned}
A\left(f_{1}, \ldots, f_{2 N}\right)= & \frac{H_{12}\left(f_{1}\right)}{P_{1}\left(\left|f_{1}\right|\right) P_{2}\left(\left|f_{2}\right|\right)} \delta_{T}\left(f_{1}+f_{2}\right) \\
& \times \frac{H_{34}\left(f_{3}\right)}{P_{3}\left(\left|f_{3}\right|\right) P_{4}\left(\left|f_{4}\right|\right)} \delta_{T}\left(f_{3}+f_{4}\right) \\
& \times \cdots \frac{H_{2 N-1,2 N}\left(f_{2 N-1}\right)}{P_{2 N-1}\left(\left|f_{2 N-1}\right|\right) P_{2 N}\left(\left|f_{2 N}\right|\right)} \\
& \times \delta_{T}\left(f_{2 N-1}+f_{2 N}\right) \\
& + \text { all possible permutations. }
\end{aligned}
$$

(v) The squared signal-to-noise ratio for the optimally filtered $2 \mathrm{~N}$-detector correlation is given by

$$
\begin{aligned}
\mathrm{SNR}_{\mathrm{optimal}}^{2} & { }^{(12)} \mathrm{SNR}^{2(34)} \mathrm{SNR}^{2} \ldots{ }^{(2 N-1,2 N)} \mathrm{SNR}^{2} \\
& + \text { all possible permutations. }
\end{aligned}
$$

(vi) The minimum value of $\Omega_{0}$ required to detect a stochastic gravity-wave signal $95 \%$ of the time, with a false alarm rate equal to $5 \%$, from data obtained via a $2 \mathrm{~N}$-detector correlation experiment, is given by

\footnotetext{
${ }^{41}$ The number of terms on the RHS of the "factorization" equation is given by $(2 N-1)(2 N-3) \cdots 1$. For $N=1,2,3, \ldots$, this corresponds to $1,3,15, \ldots$ terms.
} 


$$
\begin{aligned}
\left(\left.\Omega_{0}^{95 \%, 5 \%}\right|_{\text {optimal }}\right)^{-2}= & (2 \times 1.65)^{(2 N-2) / N} \\
& \times\left[\left({ }^{(12)} \Omega_{0}^{95 \%, 5 \%}\right)^{-2}\left({ }^{(34)} \Omega_{0}^{95 \%, 5 \%}\right)^{-2}\right. \\
& \times \cdots\left({ }^{(2 N-1,2 N)} \Omega_{0}^{95 \%, 5 \%}\right)^{-2} \\
& + \text { all possible permutations }]^{1 / N}
\end{aligned}
$$

where ${ }^{(i j)} \Omega_{0}^{95 \%, 5 \%}$ are the analogous quantities for the $i j$ detector pairs.

\section{E. Correlated detector noise}

We have shown how to carry out an experimental search for a stochastic background of gravitational radiation, by correlating the outputs of widely separated detectors. Our analysis assumed that any correlation between the two outputs arises only from a stochastic gravity-wave background. In this section, we address the validity of this assumption, and look at possible sources of instrumental contamination that give rise to a correlated signal between the separated detectors. Any source of correlated environmental noise or interference in the separated detectors mimics the correlation arising from a stochastic background; so it is important to understand the order-of-magnitude effects of any potential sources of such correlated noise.

This subject has already been considered in some detail, both in the the published paper of Christensen [6] and, in more detail, in Chap. 7 of his Ph.D. thesis [24]. In the thesis work, the following sources of correlated detector noise are analyzed:

(i) Seismic noise, whose effects on initial LIGO are minimal.

(ii) Fluctuations in the residual gas (for two interferometers sharing a common vacuum system such as the LIGO-WA site). The effects on initial LIGO are minimal, and of course there is no effect for separated detectors that do not share a common vacuum envelope.

(iii) Acoustic noise, whose effects on LIGO are minimal.

(iv) Cosmic ray showers, whose effects on LIGO are minimal.

(v) Magnetic field fluctuations, whose effects on initial LIGO might be significant.

In this section, we derive a general formalism for calculating the effects of such correlated detector noise, and illustrate this for the case of correlated magnetic fields, which Christensen concluded would be the most significant source of correlated fluctuations at two widely separated sites.

Although these ideas can be generalized to multiple site locations, for simplicity we present only the two-site case. Correlated noise in two detectors can be described by the cross-spectral function $C(f)$ defined by

$$
\left\langle n_{1}(t) n_{2}\left(t^{\prime}\right)\right\rangle=\frac{1}{2} \int_{-\infty}^{\infty} d f e^{i 2 \pi f\left(t-t^{\prime}\right)} C(f) .
$$

Because the LHS is real, the cross-spectrum satisfies $C(f)$ $=C^{*}(-f)$. If this cross spectrum is non-vanishing, then it produces a correlation between the two detectors which mimics the effect of a stochastic gravity-wave background.

It is straightforward to determine the point at which a non-vanishing cross spectrum $C(f)$ will significantly interfere with a stochastic background search. Such interference will not take place if the correlation arising from the cross spectrum of detector noise is significantly less than that arising from the stochastic gravity-wave background:

$$
\left\langle n_{1}(t) n_{2}\left(t^{\prime}\right)\right\rangle \ll\left\langle h_{1}(t) h_{2}\left(t^{\prime}\right)\right\rangle \text { for }\left|t-t^{\prime}\right| \lesssim d / c,
$$

where $d / c$ is the light travel time between the two sites. Making use of Eqs. (5.107) and (5.74), we see that correlated detector noise will not impair a stochastic background search if

$$
\frac{1}{2}|C(f)| \ll \frac{3 H_{0}^{2}}{20 \pi^{2}}|f|^{-3} \Omega_{\mathrm{gw}}(|f|) \gamma(|f|)
$$

over the range of frequencies included in the optimal filter $Q(f)$. For example, for the initial LIGO detectors, this range of frequencies is from about $40 \mathrm{~Hz}$ to $300 \mathrm{~Hz}$, and in a 4 month search, the expected level of sensitivity is about $\Omega_{0} h_{100}^{2} \sim 10^{-6}$; so the RHS is $\sim 3 \times 10^{-49} h_{100}^{2}$ sec. Thus, in order that correlated sources of noise do not interfere with initial LIGO's 4-month stochastic background search, one must have

$$
|C(f)| \ll 3 \times 10^{-49} h_{100}^{2} \text { sec for } 40 \mathrm{~Hz}<f<300 \mathrm{~Hz} \text {. }
$$

This limit can be stated in an interesting way. If we compare the allowable cross spectrum $C(f)$ with the intrinsic noise power spectrum $P(f) \sim 10^{-45}$ sec in each detector, we see that they differ by about four orders of magnitude. Thus, in order that correlated noise sources do not interfere with a 4-month long stochastic background search for initial LIGO, the correlated sources of noise must not contribute more than $1 \%$ of the motion of the test masses, in the frequency range from $40 \mathrm{~Hz}$ to $300 \mathrm{~Hz}$.

Essentially the same limit on correlated noise can be written in another fashion. If we make use of Eq. (3.52), the contribution of correlated noise to the expected mean of the signal can be written as

$$
\mu_{\text {correlated noise }}^{2}=\left|\frac{1}{2} T \int_{-\infty}^{\infty} d f C(f) \widetilde{Q}(f)\right|^{2} .
$$

Requiring that this be smaller than the magnitude of the variance $\sigma^{2}$ [see Eq. (3.68)] leads immediately to the condition that the correlated noise will not interfere with a stochastic background search over an observation time $T$ if

$$
|C(f)|^{2} \ll \frac{P_{1}(f) P_{2}(f)}{T f} .
$$

As before, the contribution of correlated noise to the motion of the interferometer must be smaller than the intrinsic detector noise motion by a factor of $(T f)^{-1 / 2}$. For an observa- 
tion time $T$ of 4 months (i.e., $10^{7} \mathrm{sec}$ ) and frequencies $f$ $\sim 100 \mathrm{~Hz}$, this gives the same $1 \%$ bound as before.

One can also give a precise formula showing the effects of correlated noise sources on the expected value $\mu$ of the signal. Making use of Eqs. (5.11) and (3.57), we can express the ratio of the signal mean arising from the correlated noise to that arising from the stochastic background as

$$
\begin{aligned}
& \left|\frac{\mu_{\text {correlated noise }}}{\mu_{\text {stochastic background }}}\right| \\
& =\frac{10 \pi^{2}}{3 H_{0}^{2}}\left|\frac{\int_{-\infty}^{\infty} d f C(f) \widetilde{Q}(f)}{\int_{-\infty}^{\infty} d f|f|^{-3} \Omega_{\mathrm{gw}}(|f|) \gamma(|f|) \widetilde{Q}(f)}\right| .
\end{aligned}
$$

This formula allows us to precisely determine the effect of any correlated source of noise on the signal value starting from a model or measurement of the correlation spectrum $C(f)$. In particular, if we assume that the stochastic gravitywave background has a constant frequency spectrum $\Omega_{\mathrm{gw}}(f)=\Omega_{0}$, then

$$
\left|\frac{\mu_{\text {correlated noise }}}{\mu_{\text {stochastic background }}}\right|=\frac{\Omega_{\text {correlated noise limit }}}{\Omega_{0}},
$$

where $\Omega_{\text {correlated noise limit }}$ is (by definition) the smallest value of $\Omega_{0}$ that can be observed before the effects of correlated instrument noise interfere with the measurement.

The effects of a given source of correlated noise can be modeled more precisely. Here, we work through one example: the effects of correlated magnetic field fluctuations. Christensen's work $[6,24]$ concludes that these are the most likely environmental source of correlated noise between two sites.

The LIGO interferometers use small magnets to steer and push the optical elements (i.e., mirrors and beam splitters). These magnets are an integral part of the Length Sensing and Control (LSC) system. Forces are applied to these magnets with electromagnetic coils, and they are part of the servo loop that uses modulation techniques to lock and monitor the path length difference between the test masses. External magnetic fields, present in the environment, exert forces on these magnets thus constituting one of the different sources of instrument noise.

The external magnetic field is of particular concern, because it propagates at the speed of light, and therefore can give rise to correlations between sites on the time scale $d / c$. The magnetic field in the laboratory consists of two parts: a "local" part and a "global" part. The local part is the magnetic field arising from the instrumentation, wiring, and power lines within the laboratory; the global part comes from Schuman resonances of the Earth and the ionosphere, and lightning strikes over the surface of the Earth. The local part of the magnetic field will not correlate between widely separated sites; the global part, however, is likely to be highly correlated. Studies in the Caltech 40-m prototype laboratory [25] have shown that the spectrum of ambient magnetic fields is of order $10^{-7} \mathrm{G} / \sqrt{\mathrm{Hz}}$, but most of this field is local. Christensen reports in his thesis on a number of studies that show that the global magnetic fields are well-modeled above $20 \mathrm{~Hz}$ by a power-law spectrum:

$$
P_{\mathrm{B}}(f)=A\left(\frac{f}{40 \mathrm{~Hz}}\right)^{-0.88}
$$

with $A \sim 1.2 \times 10^{-17} \mathrm{G}^{2} / \mathrm{Hz}$ during magnetically noisy periods such as thunderstorms and $A \sim 1.8 \times 10^{-19} \mathrm{G}^{2} / \mathrm{Hz}$ during magnetically quiet periods. Separate measurements have shown that these fields have a coherence of order $r \sim 1 / 2$ in the frequency range of interest, over widely separated (almost antipodal) points on the Earth's surface. These highly correlated global fields are the main concern here.

In order to reduce the effects of external magnetic fields on the test masses and optics, the four magnets on each optic or test mass are arranged to approximately cancel both the dipole and quadrupole parts of the magnetic field. The magnitude of the resulting force on a test mass may be described by

$$
F=\frac{\mu B}{l}\left[\epsilon_{0}+\frac{\Delta}{l} \epsilon_{1}+\frac{\Delta^{2}}{l^{2}} \epsilon_{2}+\cdots\right] .
$$

Here, $\mu$ is the magnetic dipole moment of one of the magnets, $B$ is the magnitude of the ambient magnetic field, $l$ is the length scale over which the magnetic field is varying in the vicinity of the test masses and optics, and $\Delta$ is the separation between the magnets on the test masses and optics. The quantities $\epsilon_{0}, \epsilon_{1}, \ldots$ are the fractional difference between the dipole, quadrupole, ... moments of the different magnets (which are not perfectly matched). For the initial LIGO detectors, the preliminary design values of these quantities are approximately $\mu=0.11 \mathrm{~A} \mathrm{~m}^{2} / c, \epsilon_{0}=0.05$, $l=6 \mathrm{~cm}, \Delta=15 \mathrm{~cm}$. This leads to a force

$$
F=\kappa B
$$

on the test masses, with $\kappa \sim 0.1 \mathrm{dyn} / \mathrm{G}$ [25]. This force accelerates the test mass, producing an equivalent strain

$$
\tilde{n}(f) \sim \frac{\widetilde{F}(f)}{M(2 \pi f)^{2} L},
$$

where $M=10 \mathrm{~kg}$ is the mass of the optic, and $L=4 \mathrm{~km}$ is the length of the arm. This gives rise to a cross spectrum

$$
C(f)=\frac{r \kappa^{2}}{M^{2}(2 \pi f)^{4} L^{2}} P_{B}(f),
$$

where $r$ is the coherence. Evaluating the integral in Eqs. (5.113) and (5.114) we find that the limits on $\Omega_{0}$ are 


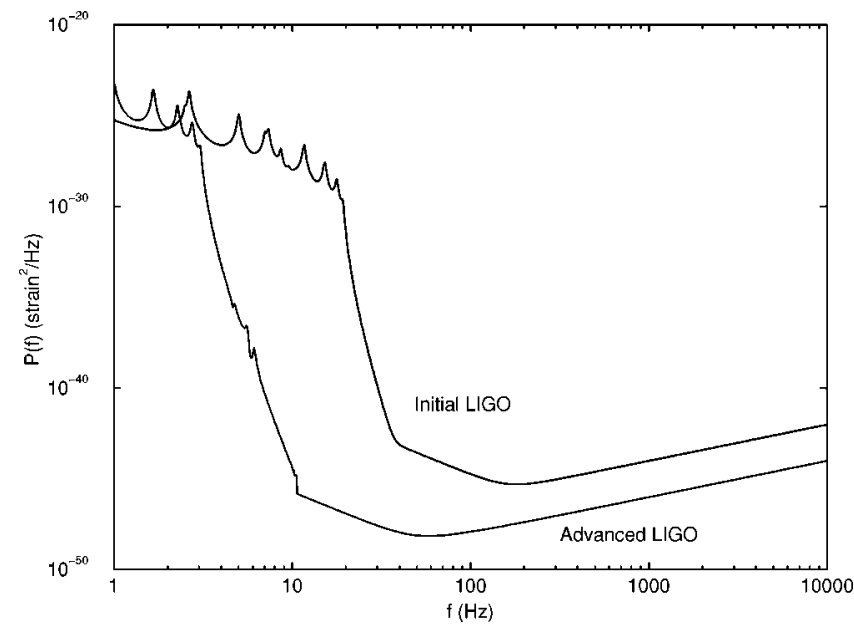

FIG. 10. A log-log plot of the predicted noise power spectra for the initial and advanced LIGO detectors.

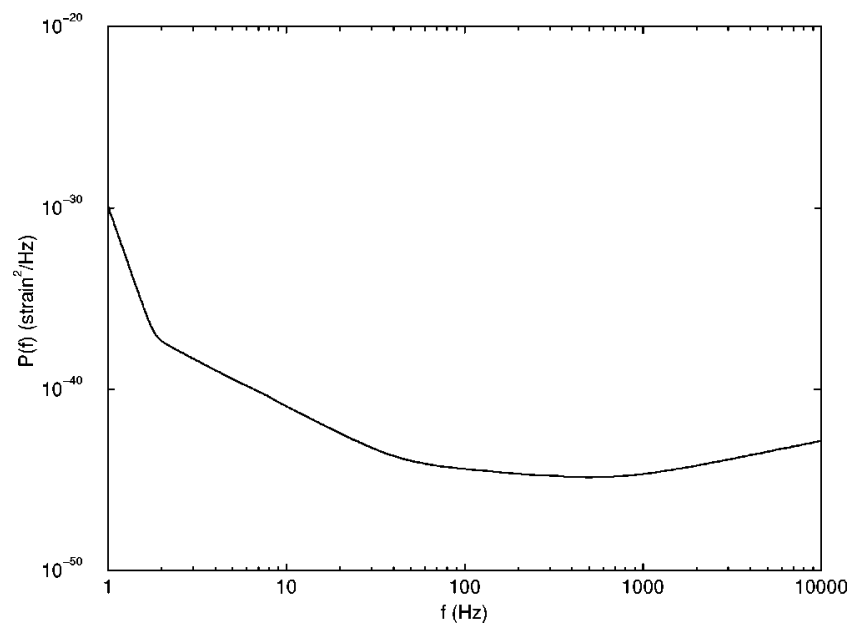

FIG. 11. A log-log plot of the predicted noise power spectrum for the VIRGO detector.

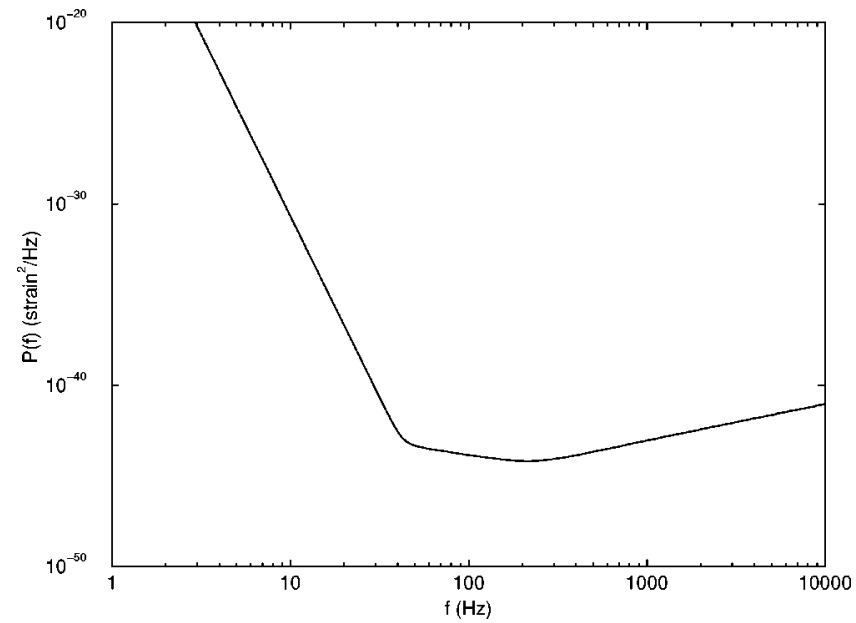

FIG. 12. A log-log plot of the predicted noise power spectrum for the GEO-600 detector.

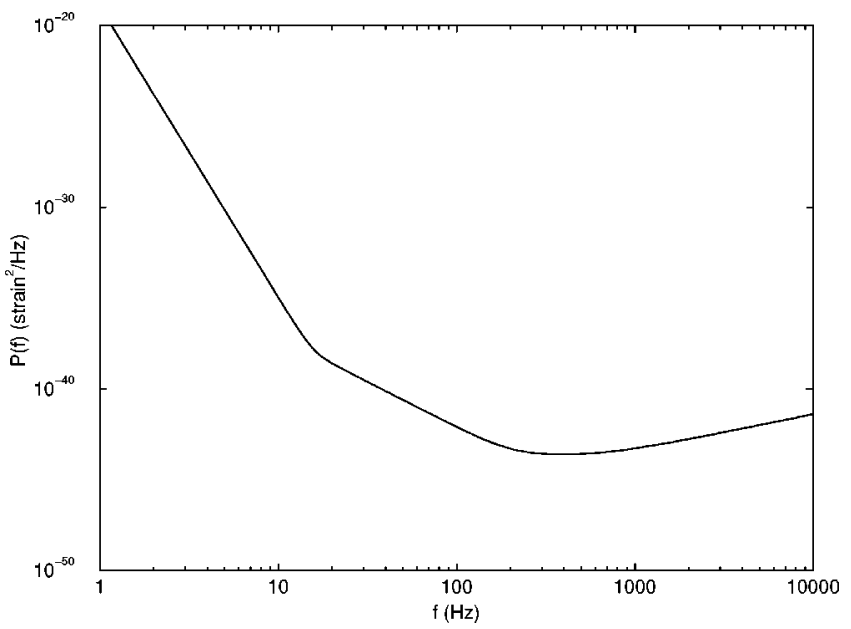

FIG. 13. A log-log plot of the predicted noise power spectrum for the TAMA-300 detector.

$\Omega_{\text {correlated noise limit }}$

$$
= \begin{cases}10^{-7} & \text { during magnetically “"noisy" times, } \\ 1.5 \times 10^{-9} & \text { during magnetically " "quiet” times. }\end{cases}
$$

Our conclusion is that for the initial LIGO design (where sensitivities are on the order of $6 \times 10^{-6}$ for 4 months of observation), magnetic field induced correlations are not a concern. However, for advanced LIGO (where sensitivities are on the order of $6 \times 10^{-11}$ for 4 months of observation), the magnets must be eliminated from the design or they will they will significantly constrain the measurements of (or limits placed on) $\Omega_{0}$.

\section{NUMERICAL DATA}

This section consists of a series of graphs and tables containing numerical data for the five major interferometers: (i) Hanford, WA LIGO detector (LIGO-WA), (ii) Livingston, LA LIGO detector (LIGO-LA), (iii) VIRGO detector

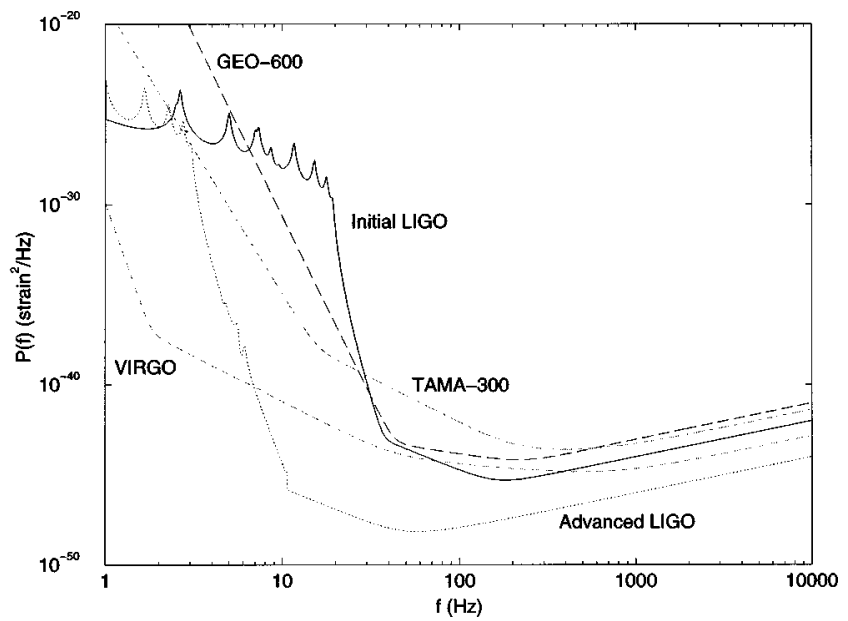

FIG. 14. A log-log plot of the predicted noise power spectra for all the major interferometers. 


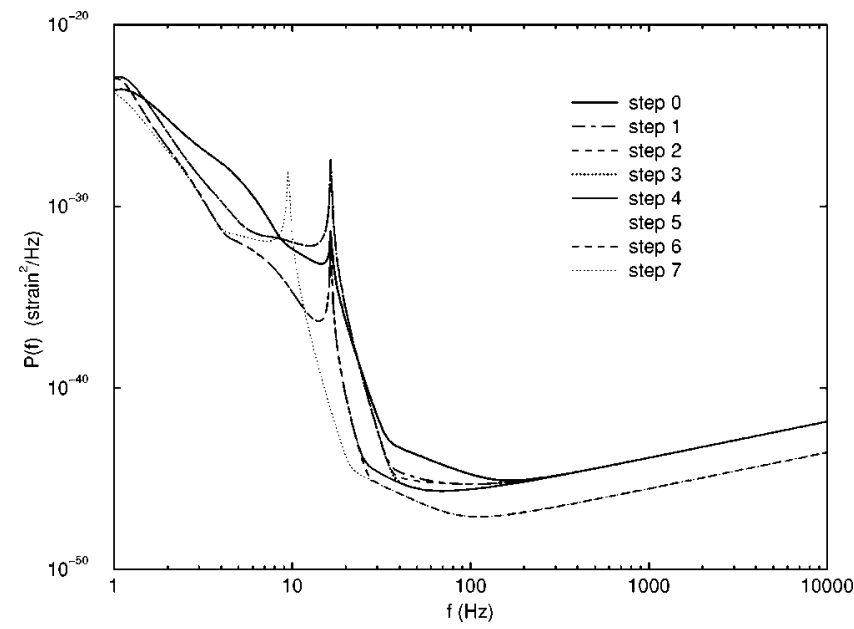

FIG. 15. A log-log plot of the predicted noise power spectra for the "enhanced" LIGO detectors, showing the probable evolution of the detector design over the next decade.

(VIRGO), (iv) GEO-600 detector (GEO-600), and (v) TAMA-300 detector (TAMA-300). These data were derived from published site location and orientation information and detector noise power spectra design goals [26], using the stochastic background data analysis routines contained in GRASP [27]. (See Sec. VII for more information about the computer code that we wrote to perform these calculations.)

\section{A. Noise power spectra}

Figures 10-13 show the predicted noise power spectra for the initial and advanced LIGO detectors, and for the VIRGO, GEO-600, and TAMA-300 detectors. Figure 14 displays all of the noise power spectra on a single graph. Figure 15 shows the predicted noise power spectra for the "enhanced" LIGO detectors, which track the projected performance of the LIGO detector design over the next decade. The data for

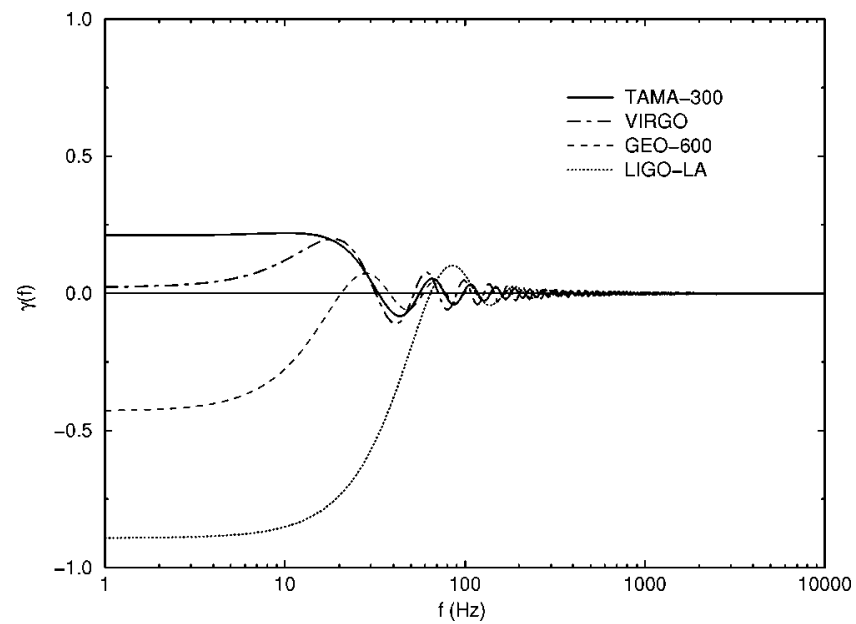

FIG. 16. The overlap reduction function $\gamma(f)$ for the LIGO-WA detector and the other major interferometers. Note that the overlap reduction functions for the more distant detectors have their first zero at lower frequencies than those for the more nearby detectors.

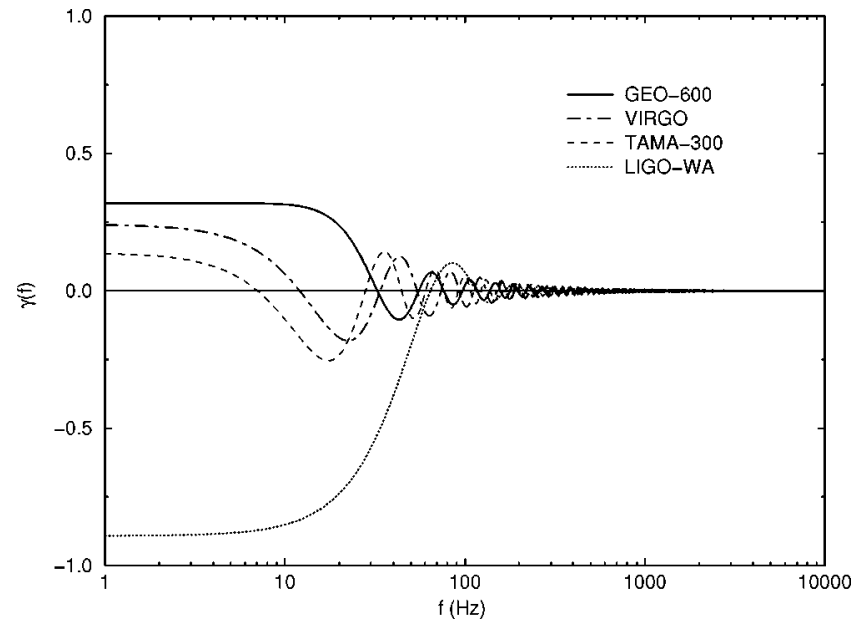

FIG. 17. The overlap reduction function $\gamma(f)$ for the LIGO-LA detector and the other major interferometers.

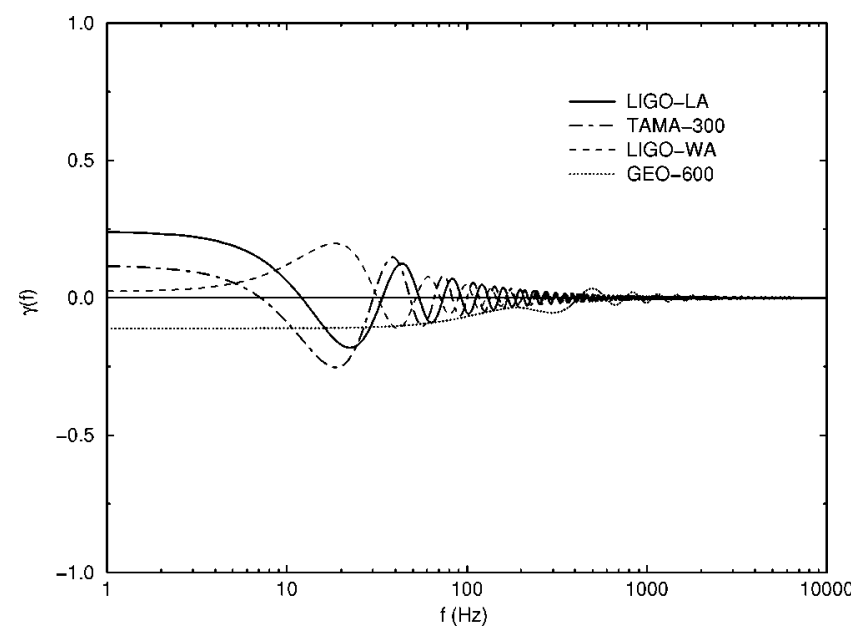

FIG. 18. The overlap reduction function $\gamma(f)$ for the VIRGO detector and the other major interferometers. Note that the VIRGO and GEO-600 detectors are sensitive to almost orthogonal polarizations.

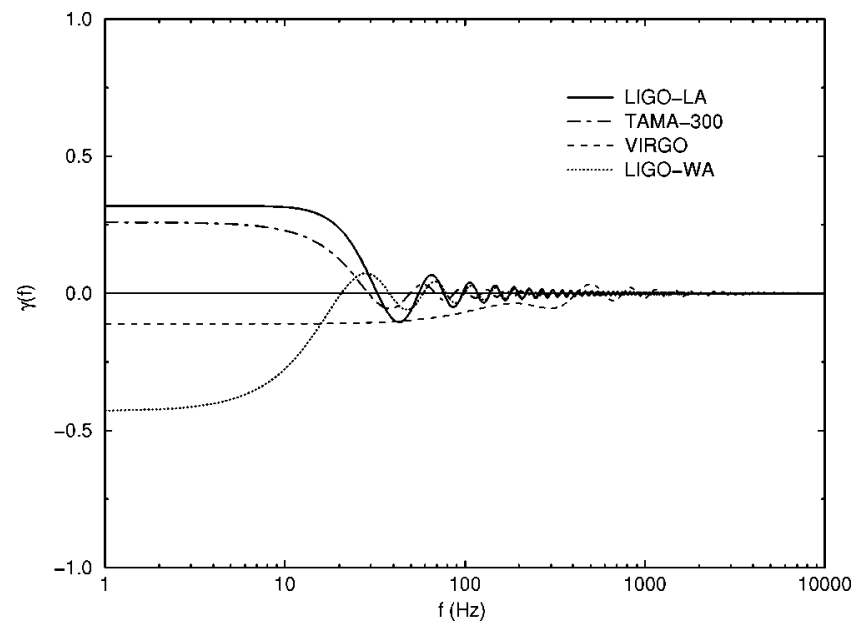

FIG. 19. The overlap reduction function $\gamma(f)$ for the GEO-600 detector and the other major interferometers. Note that the VIRGO and GEO-600 detectors are sensitive to almost orthogonal polarizations. 


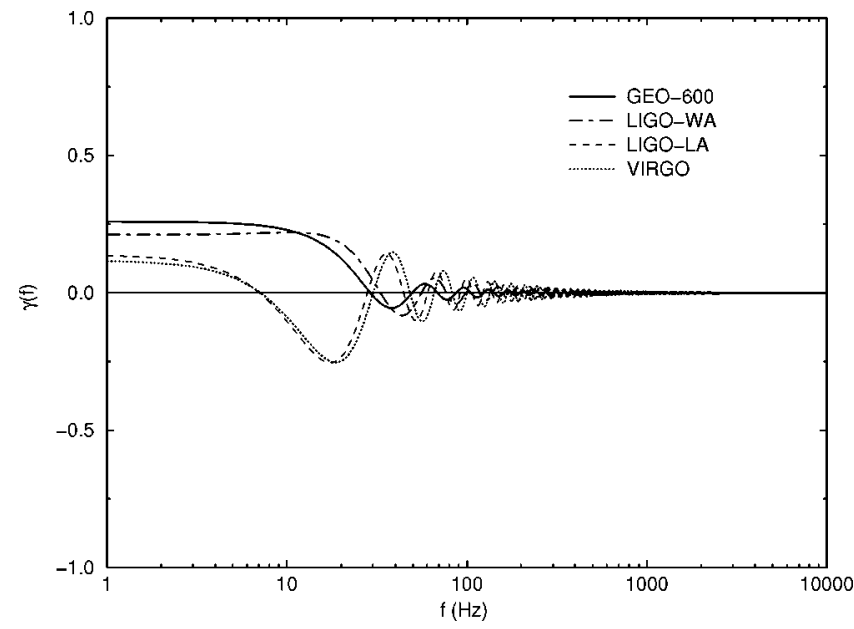

FIG. 20. The overlap reduction function $\gamma(f)$ for the TAMA300 detector and the other major interferometers.

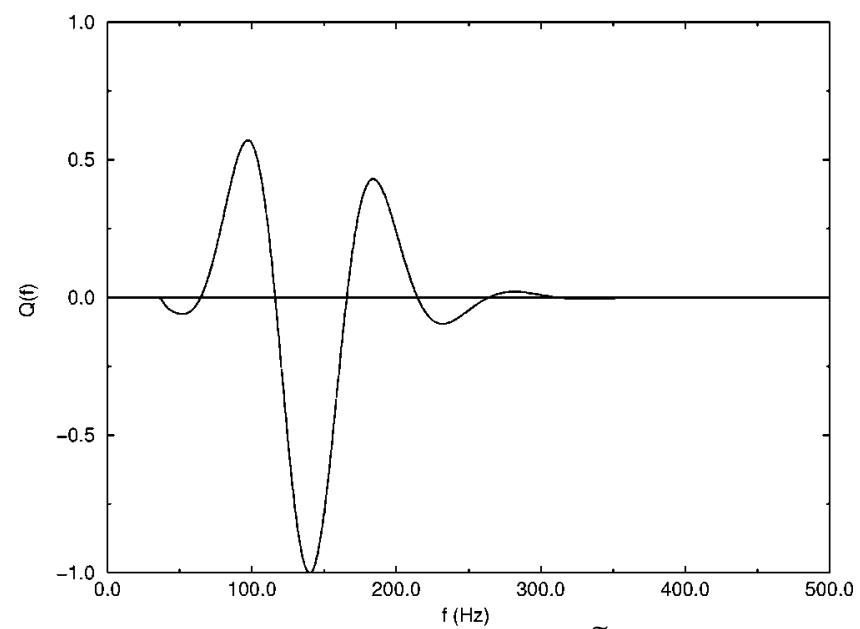

FIG. 21. The optimal filter function $\widetilde{Q}(f)$ for the initial LIGO-WA and initial LIGO-LA detectors, for a stochastic background having a constant frequency spectrum $\Omega_{\mathrm{gw}}(f)=\Omega_{0}$. The optimal filter function is normalized to have maximum magnitude of unity.

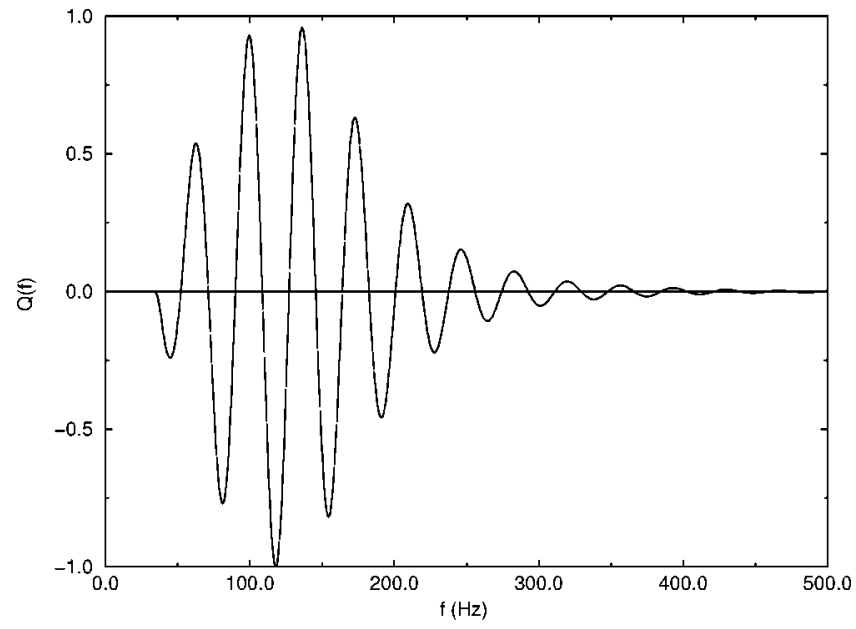

FIG. 22. The optimal filter function $\widetilde{Q}(f)$ for the initial LIGO-WA and VIRGO detectors, for a stochastic background having a constant frequency spectrum $\Omega_{\mathrm{gw}}(f)=\Omega_{0}$. The optimal filter function is normalized to have maximum magnitude of unity.

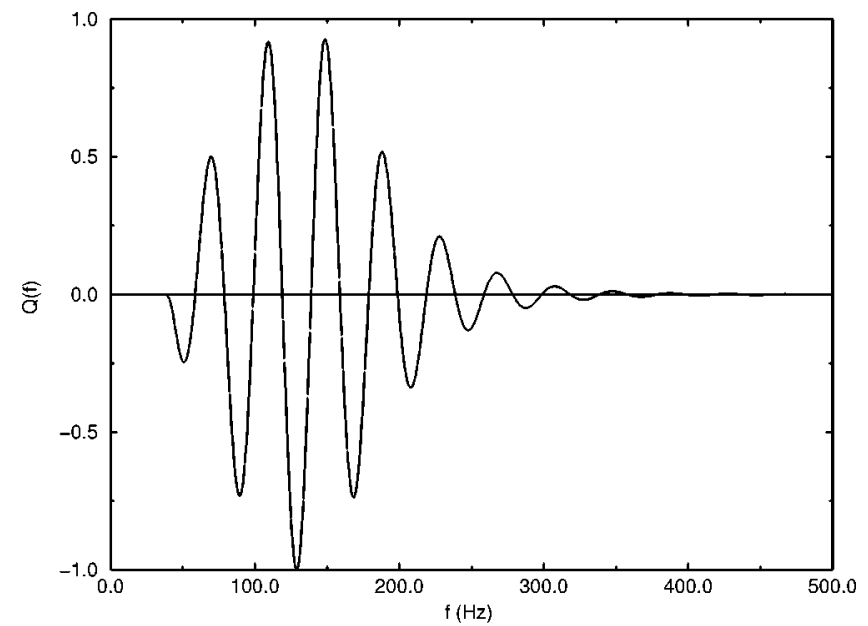

FIG. 23. The optimal filter function $\widetilde{Q}(f)$ for the initial LIGO-WA and GEO-600 detectors, for a stochastic background having a constant frequency spectrum $\Omega_{\mathrm{gw}}(f)=\Omega_{0}$. The optimal filter function is normalized to have maximum magnitude of unity.

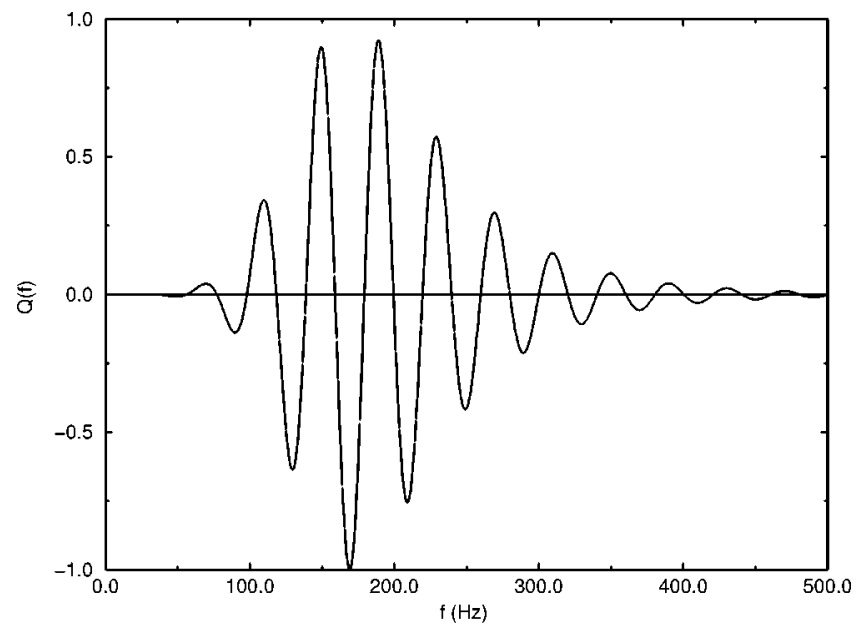

FIG. 24. The optimal filter function $\widetilde{Q}(f)$ for the initial LIGO-WA and TAMA-300 detectors, for a stochastic background having a constant frequency spectrum $\Omega_{\mathrm{gw}}(f)=\Omega_{0}$. The optimal filter function is normalized to have maximum magnitude of unity.

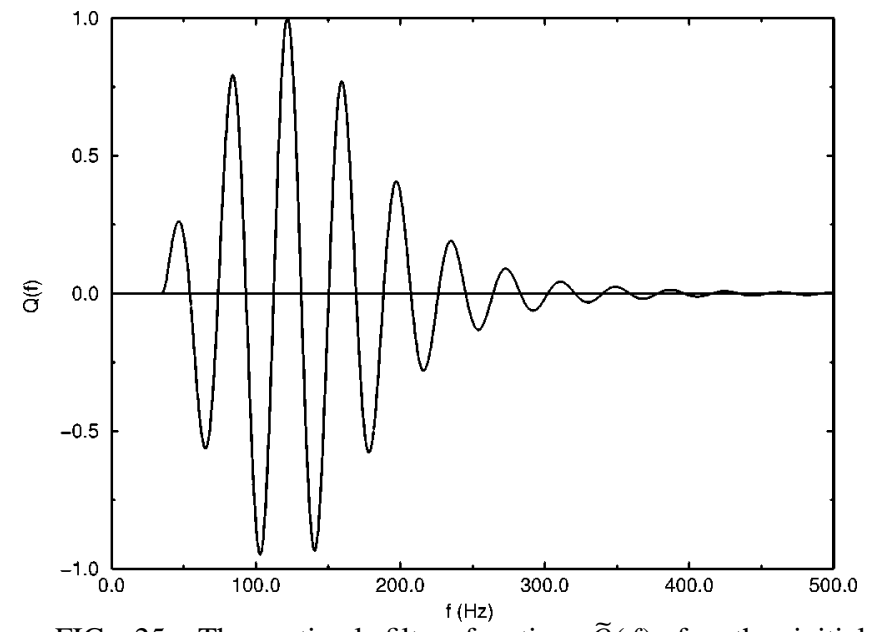

FIG. 25. The optimal filter function $\widetilde{Q}(f)$ for the initial LIGO-LA and VIRGO detectors, for a stochastic background having a constant frequency spectrum $\Omega_{\mathrm{gw}}(f)=\Omega_{0}$. The optimal filter function is normalized to have maximum magnitude of unity. 


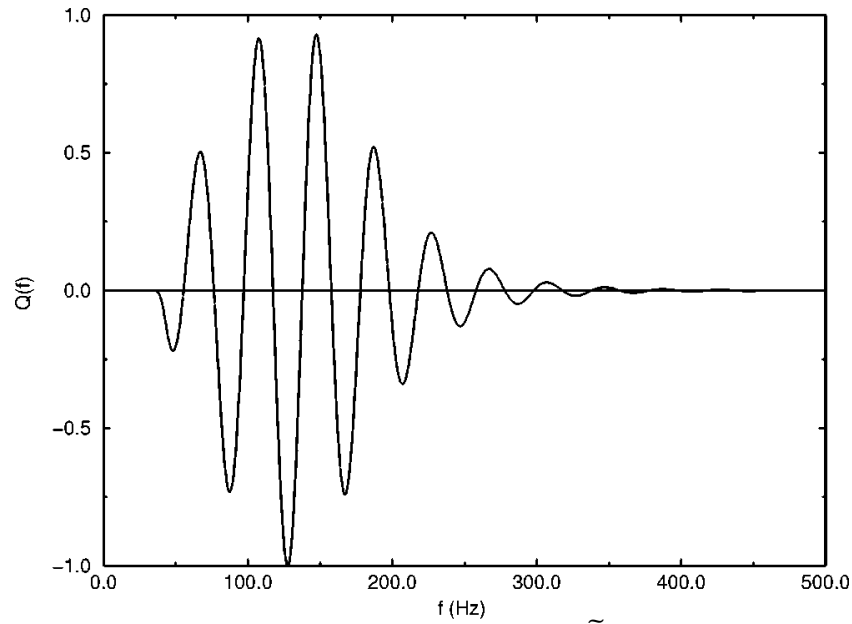

FIG. 26. The optimal filter function $\widetilde{Q}(f)$ for the initial LIGO-LA and GEO-600 detectors, for a stochastic background having a constant frequency spectrum $\Omega_{\mathrm{gw}}(f)=\Omega_{0}$. The optimal filter function is normalized to have maximum magnitude of unity.

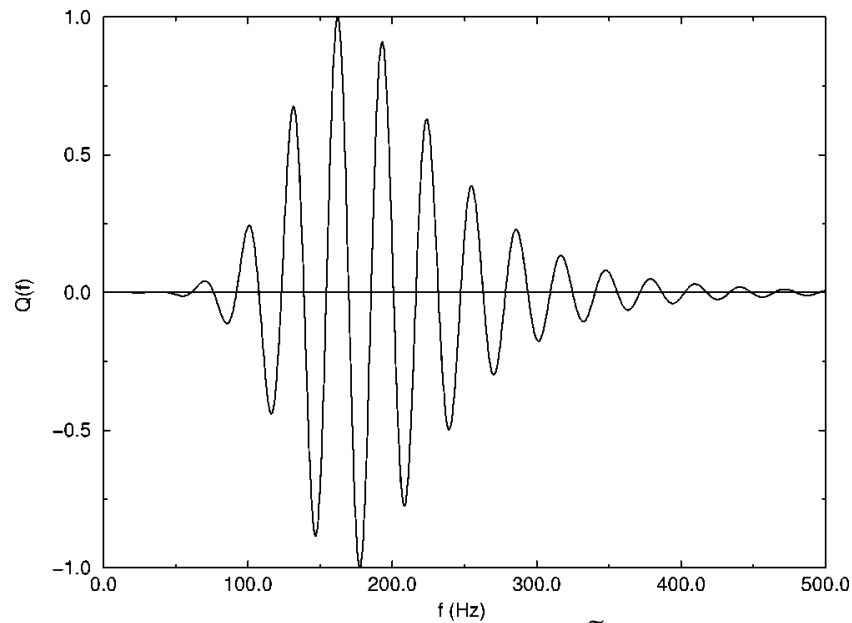

FIG. 27. The optimal filter function $\widetilde{Q}(f)$ for the initial LIGO-LA and TAMA-300 detectors, for a stochastic background having a constant frequency spectrum $\Omega_{\mathrm{gw}}(f)=\Omega_{0}$. The optimal filter function is normalized to have maximum magnitude of unity.

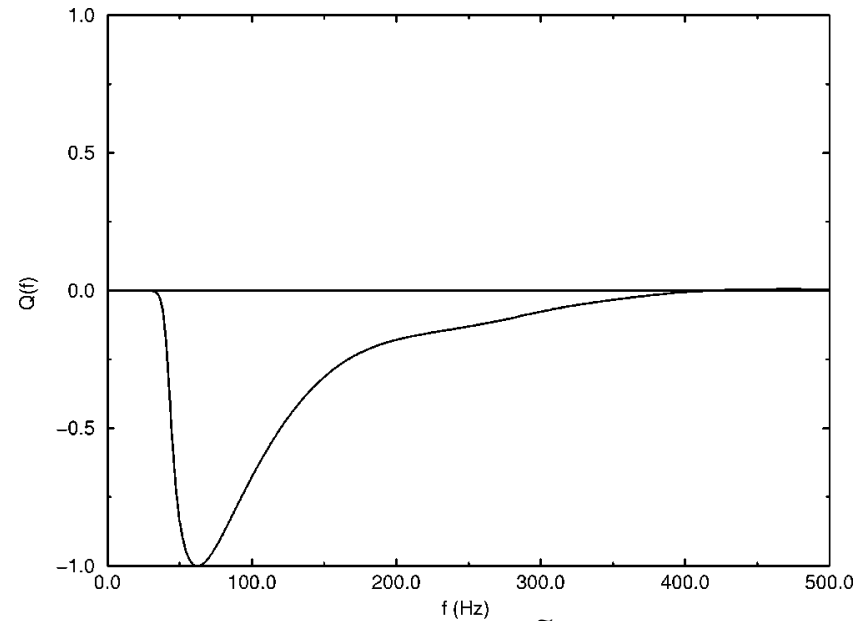

FIG. 28. The optimal filter function $\widetilde{Q}(f)$ for the VIRGO and GEO-600 detectors, for a stochastic background having a constant frequency spectrum $\Omega_{\mathrm{gw}}(f)=\Omega_{0}$. The optimal filter function is normalized to have maximum magnitude of unity.

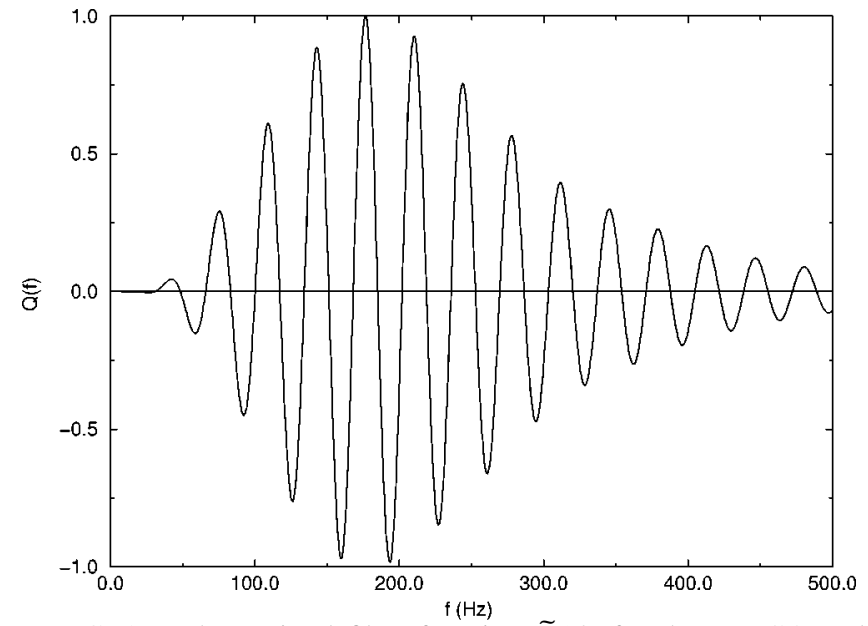

FIG. 29. The optimal filter function $\widetilde{Q}(f)$ for the VIRGO and TAMA-300 detectors, for a stochastic background having a constant frequency spectrum $\Omega_{\mathrm{gw}}(f)=\Omega_{0}$. The optimal filter function is normalized to have maximum magnitude of unity.

the noise power spectra displayed in all of these figures were taken from the published design goals [26].

\section{B. Overlap reduction functions}

Figures 16-20 show the overlap reduction functions $\gamma(f)$ for different detector pairs.

\section{Optimal filter functions}

Figures 21-30 show the optimal filter functions $\widetilde{Q}(f)$ for different detector pairs, for a stochastic background having a constant frequency spectrum $\Omega_{\mathrm{gw}}(f)=\Omega_{0}$. The optimal filter functions are normalized to have maximum magnitude of unity.

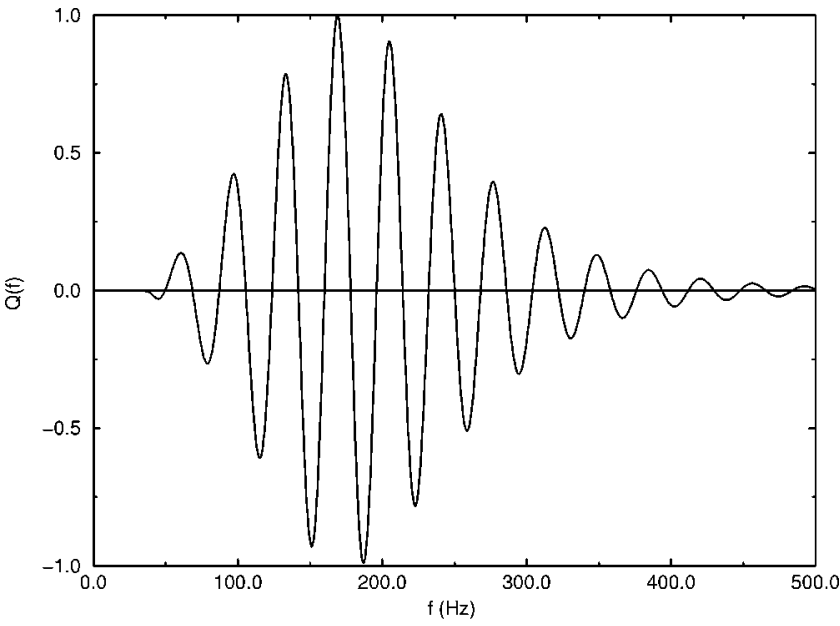

FIG. 30. The optimal filter function $\widetilde{Q}(f)$ for the GEO-600 and TAMA-300 detectors, for a stochastic background having a constant frequency spectrum $\Omega_{\mathrm{gw}}(f)=\Omega_{0}$. The optimal filter function is normalized to have maximum magnitude of unity. 
TABLE I. Theoretical signal-to-noise ratios after 4 months of observation, for the optimally-filtered cross-correlation signal between different detector pairs, for a stochastic background of gravitational radiation having a constant frequency spectrum $\Omega_{\mathrm{gw}}(f)=\Omega_{0}=6 \times 10^{-6} h_{100}^{-2}$.

\begin{tabular}{lccccc}
\hline \hline & LIGO-WA & LIGO-LA & VIRGO & GEO-600 & TAMA-300 \\
\hline LIGO-WA & - & 3.45 & 1.74 & $5.09 \times 10^{-1}$ & $6.12 \times 10^{-2}$ \\
LIGO-LA & 3.45 & - & 2.10 & $7.66 \times 10^{-1}$ & $9.16 \times 10^{-2}$ \\
VIRGO & 1.74 & 2.10 & - & 1.56 & $9.14 \times 10^{-2}$ \\
GEO-600 & $5.09 \times 10^{-1}$ & $7.66 \times 10^{-1}$ & 1.56 & - & $1.32 \times 10^{-2}$ \\
TAMA-300 & $6.12 \times 10^{-2}$ & $9.16 \times 10^{-2}$ & $9.14 \times 10^{-2}$ & $1.32 \times 10^{-2}$ & - \\
\hline \hline
\end{tabular}

\section{Signal-to-noise ratios and sensitivities}

Table I contains the values of the theoretical signal-tonoise ratios after 4 months (i.e., $10^{7} \mathrm{sec}$ ) of observation, for the optimally filtered cross-correlation signal between different detector pairs, for a stochastic background of gravitational radiation having a constant frequency spectrum $\Omega_{\mathrm{gw}}(f)=\Omega_{0}=6 \times 10^{-6} h_{100}^{-2}$. Table II contains the minimum values of $\Omega_{0} h_{100}^{2}$ for 4 months of observation, for a false alarm rate equal to $5 \%$, and for a detection rate equal to $95 \%$, for cross-correlation measurements between different detector pairs. Tables III and IV contain the minimum values $\Omega_{0} h_{100}^{2}$ for 4 months of observation, for a false alarm rate equal to $5 \%$, and for a detection rate equal to $95 \%$, for the optimal combination of cross-correlation measurements between multiple detector pairs, taken from all possible triples and quadruples of the five major interferometers. Table $\mathrm{V}$ contains the minimum values of $\Omega_{0} h_{100}^{2}$ for 4 months of observation, for a false alarm rate equal to $5 \%$, and for a detection rate equal to $95 \%$, for optimally filtered 4-detector correlations. [Note that the calculation of the signal-to-noise ratios and minimum values of $\Omega_{0}$ assumes that the magnitude of the noise intrinsic to the detectors is much larger than the stochastic gravity-wave background. This corresponds to Eq. (3.75) in the text.]

\section{COMPUTER SIMULATION}

In Secs. III-V, we described the data analysis and optimal signal processing required for the detection of a stochastic background of gravitational radiation. This analysis was in terms of continuous time functions and their associated Fourier transforms. But, in reality, when one performs the actual data analysis, continuous time functions will be replaced by discrete time series and Fourier transforms by their discrete frequency counterparts. The discrete data can then be processed by computer code that takes the appropriate FFTs, constructs the optimal filters, whitens and windows the data, etc. We have written a number of functions (in ANSI-C) to do precisely this. These functions constitute part of a generalpurpose data analysis package for gravitational-wave detection, called GRASP (Gravitational Radiation Analysis and Simulation Package) [27]. In this section, we describe a computer simulation (made up of these functions) that mimics the generation and detection of a simulated stochastic gravity-wave signal in the presence of simulated detector noise. Documentation and further information about the code can be found in the GRASP user's manual.

\section{A. Purpose}

The main reason for writing the computer simulation was to verify many of the theoretical calculations that were derived in the previous sections. Specifically, we wanted to see if the theoretically predicted signal-to-noise ratio SNR, for a stochastic background of gravitational radiation having a constant frequency spectrum $\Omega_{\mathrm{gw}}(f)=\Omega_{0}$, would agree with an "experimentally" determined signal-to-noise ratio $\widehat{\mathrm{SNR}}$ produced by the simulation. If the theoretical and experimental signal-to-noise ratios agreed, we could be confident that the theoretical calculations were correct. If they did not agree, we would know that something-either a theoretical calculation or a technical issue related to the simulation itself-needed further investigation.

The theoretical and experimental signal-to-noise ratios were said to be in agreement if the relative error defined by

TABLE II. Minimum values of $\Omega_{0} h_{100}^{2}$ for 4 months of observation, for a false alarm rate equal to $5 \%$, and for a detection rate equal to $95 \%$, for cross-correlation measurements between different detector pairs.

\begin{tabular}{lccccc}
\hline \hline & LIGO-WA & LIGO-LA & VIRGO & GEO-600 & TAMA-300 \\
\hline LIGO-WA & - & $5.74 \times 10^{-6}$ & $1.14 \times 10^{-5}$ & $3.89 \times 10^{-5}$ & $3.24 \times 10^{-4}$ \\
LIGO-LA & $5.74 \times 10^{-6}$ & - & $9.45 \times 10^{-6}$ & $2.58 \times 10^{-5}$ & $2.16 \times 10^{-4}$ \\
VIRGO & $1.14 \times 10^{-5}$ & $9.45 \times 10^{-6}$ & - & $1.27 \times 10^{-5}$ & $2.17 \times 10^{-4}$ \\
GEO-600 & $3.89 \times 10^{-5}$ & $2.58 \times 10^{-5}$ & $1.27 \times 10^{-5}$ & - & $1.50 \times 10^{-3}$ \\
TAMA-300 & $3.24 \times 10^{-4}$ & $2.16 \times 10^{-4}$ & $2.17 \times 10^{-4}$ & $1.50 \times 10^{-3}$ & - \\
\hline \hline
\end{tabular}


TABLE III. Minimum values of $\Omega_{0} h_{100}^{2}$ for 4 months of observation, for a false alarm rate equal to $5 \%$, and for a detection rate equal to $95 \%$, for the optimal combination of cross-correlation measurements between multiple detector pairs, taken from all possible triples of the five major interferometers.

\begin{tabular}{lc}
\hline \hline Detectors & $\left.\Omega_{0}^{95 \%, 5 \%}\right|_{\text {optimal }} h_{100}^{2}$ \\
\hline LIGO-WA, LIGO-LA, VIRGO & $4.50 \times 10^{-6}$ \\
LIGO-WA, LIGO-LA, GEO-600 & $5.55 \times 10^{-6}$ \\
LIGO-WA, LIGO-LA, TAMA-300 & $5.74 \times 10^{-6}$ \\
LIGO-WA, VIRGO, GEO-600 & $8.28 \times 10^{-6}$ \\
LIGO-WA, VIRGO, TAMA-300 & $1.14 \times 10^{-5}$ \\
LIGO-WA, GEO-600, TAMA-300 & $3.86 \times 10^{-5}$ \\
LIGO-LA, VIRGO, GEO-600 & $7.27 \times 10^{-6}$ \\
LIGO-LA, VIRGO, TAMA-300 & $9.43 \times 10^{-6}$ \\
LIGO-LA, GEO-600, TAMA-300 & $2.57 \times 10^{-5}$ \\
VIRGO, GEO-600, TAMA-300 & $1.27 \times 10^{-5}$ \\
\hline \hline
\end{tabular}

$$
\text { relative error }:=\left|\frac{\widehat{\mathrm{SNR}}-\mathrm{SNR}}{\mathrm{SNR}}\right|
$$

was less than (or approximately equal to) the inverse of the theoretical signal-to-noise ratio after $n$ observation periods. ${ }^{42}$ This is the error that one would expect (approximately 68\% of the time) if we approximate the sample variance $\hat{\sigma}^{2}$ by the true variance $\sigma^{2}$, and use the fact that the sample mean

$$
\hat{\mu}:=\frac{1}{n} \sum_{i=1}^{n} S_{i}
$$

can itself be thought of as a random variable with mean $\mu$ and variance $\sigma^{2} / n$. (Recall from Sec. IV that $S_{1}, S_{2}, \ldots, S_{n}$ are $n$ statistically independent measurements of the optimally filtered cross-correlation signal $S$, each associated with one of the $n$ observation periods.) Thus,

$$
\widehat{\mathrm{SNR}}:=\frac{\hat{\mu}}{\hat{\sigma}} \approx \frac{\mu \pm \sigma / \sqrt{n}}{\sigma}=\frac{\mu}{\sigma} \pm \frac{1}{\sqrt{n}}=\mathrm{SNR} \pm \frac{1}{\sqrt{n}},
$$

which implies

$$
\text { relative error }:=\left|\frac{\widehat{\mathrm{SNR}}-\mathrm{SNR}}{\mathrm{SNR}}\right| \approx \frac{1}{\sqrt{n \mathrm{SNR}}} .
$$

This criterion was satisfied by our simulation runs for both the initial and advanced LIGO detector pairs.

\section{B. Flow chart}

A "flow chart" for the simulation is as follows:

(1) Input the parameters defining the simulation. This can be done either interactively or via "\#define" statements at

\footnotetext{
${ }^{42}$ The total observation time is $T_{\text {tot }}:=n T$, where $T$ is the duration of a single observation period. For our simulations, $T$ $=3.2768 \mathrm{sec}$.
}

TABLE IV. Minimum values of $\Omega_{0} h_{100}^{2}$ for 4 months of observation, for a false alarm rate equal to $5 \%$, and for a detection rate equal to $95 \%$, for the optimal combination of cross-correlation measurements between multiple detector pairs, taken from all possible quadruples of the five major interferometers.

\begin{tabular}{lc}
\hline \hline Detectors & $\left.\Omega_{0}^{95 \%, 5 \%}\right|_{\text {optimal }} h_{100}^{2}$ \\
\hline LIGO-WA, LIGO-LA, VIRGO, GEO-600 & $4.17 \times 10^{-6}$ \\
LIGO-WA, LIGO-LA, VIRGO, TAMA-300 & $4.50 \times 10^{-6}$ \\
LIGO-WA, LIGO-LA, GEO-600, TAMA-300 & $5.54 \times 10^{-6}$ \\
LIGO-WA, VIRGO, GEO-600, TAMA-300 & $8.28 \times 10^{-6}$ \\
LIGO-LA, VIRGO, GEO-600, TAMA-300 & $7.27 \times 10^{-6}$ \\
\hline \hline
\end{tabular}

the beginning of the simulation program. These parameters are

(i) the site identification numbers for the two detectors

(ii) the number of time-series data points $N$ to be used when performing the data analysis (i.e., FFTs, crosscorrelations, etc.). $N$ should equal an integral power of 2

(iii) the sampling period $\Delta t$ of the two detectors (Note that $T:=N \Delta t$ is the duration of a single observation period.)

(iv) the constant $\Omega_{0}$ that defines the stochastic background frequency spectrum: $\Omega_{\mathrm{gw}}(f)=\Omega_{0}$

(v) the total number of runs $n$ that make up the simulation (Note that $T_{\text {tot }}:=n T$ is the duration of the total observation period.)

(2) Using the site identification numbers, obtain site location and orientation information, and information about the noise power spectrum and whitening filter of each detector. (This information is contained in input data files.)

(3) Using the site location and orientation information, construct the overlap reduction function $\gamma\left(f_{i}\right)$ for the two detectors. [Here $f_{i}:=i /(N \Delta t)$ where $i=0,1, \ldots, N / 2-1$. By convention, we ignore the value of $\gamma(f)$, or any other function of frequency, at or above the Nyquist critical frequency $f_{\text {Nyquist }}:=1 /(2 \Delta t)$.]

(4) Simulate the generation of a stochastic background of gravitational radiation having a constant frequency spectrum $\Omega_{\mathrm{gw}}(f)=\Omega_{0}$. This can be done in the frequency domain by using a random number generator to construct (complexvalued) Gaussian random variables $\widetilde{h}_{1}\left(f_{i}\right)$ and $\widetilde{h}_{2}\left(f_{i}\right)$ having zero mean and joint expectation values:

$$
\left\langle\tilde{h}_{1}^{*}\left(f_{i}\right) \tilde{h}_{1}\left(f_{j}\right)\right\rangle=\frac{3 H_{0}^{2}}{20 \pi^{2}} T \delta_{i j} f_{i}^{-3} \Omega_{0}
$$

TABLE V. Minimum values of $\Omega_{0} h_{100}^{2}$ for 4 months of observation, for a false alarm rate equal to $5 \%$, and for a detection rate equal to $95 \%$, for optimally filtered 4-detector correlations.

\begin{tabular}{lc}
\hline \hline Detectors & $\left.\Omega_{0}^{95 \%, 5 \%}\right|_{\text {optimal }} h_{100}^{2}$ \\
\hline LIGO-WA, LIGO-LA, VIRGO, GEO-600 & $6.49 \times 10^{-6}$ \\
LIGO-WA, LIGO-LA, VIRGO, TAMA-300 & $2.51 \times 10^{-5}$ \\
LIGO-WA, LIGO-LA, GEO-600, TAMA-300 & $5.44 \times 10^{-5}$ \\
LIGO-WA, VIRGO, GEO-600, TAMA-300 & $4.68 \times 10^{-5}$ \\
LIGO-LA, VIRGO, GEO-600, TAMA-300 & $3.84 \times 10^{-5}$ \\
\hline \hline
\end{tabular}




$$
\begin{aligned}
& \left\langle\widetilde{h}_{2}^{*}\left(f_{i}\right) \widetilde{h}_{2}\left(f_{j}\right)\right\rangle=\frac{3 H_{0}^{2}}{20 \pi^{2}} T \delta_{i j} f_{i}^{-3} \Omega_{0}, \\
& \left\langle\widetilde{h}_{1}^{*}\left(f_{i}\right) \widetilde{h}_{2}\left(f_{j}\right)\right\rangle=\frac{3 H_{0}^{2}}{20 \pi^{2}} T \delta_{i j} f_{i}^{-3} \Omega_{0} \gamma\left(f_{i}\right) .
\end{aligned}
$$

These are just the discrete frequency versions of Eq. (3.56) specialized to the case $\Omega_{\mathrm{gw}}(f)=\Omega_{0}$. Note that the Fourier amplitudes $\widetilde{h}_{1}\left(f_{i}\right)$ and $\tilde{h}_{2}\left(f_{i}\right)$ fall off like $f^{-3 / 2}$.

(5) Simulate the generation of the noise intrinsic to the detectors, using the information contained in the noise power spectrum data files. This can be done in the frequency domain by using a random number generator to construct (complex-valued) Gaussian random variables $\tilde{n}_{1}\left(f_{i}\right)$ and $\tilde{n}_{2}\left(f_{i}\right)$ having zero mean and joint expectation values:

$$
\begin{aligned}
& \left\langle\tilde{n}_{1}^{*}\left(f_{i}\right) \tilde{n}_{1}\left(f_{j}\right)\right\rangle=\frac{1}{2} T \delta_{i j} P_{1}\left(f_{i}\right), \\
& \left\langle\tilde{n}_{2}^{*}\left(f_{i}\right) \tilde{n}_{2}\left(f_{j}\right)\right\rangle=\frac{1}{2} T \delta_{i j} P_{2}\left(f_{i}\right), \\
& \left\langle\tilde{n}_{1}^{*}\left(f_{i}\right) \tilde{n}_{2}\left(f_{j}\right)\right\rangle=0 .
\end{aligned}
$$

These are just the discrete frequency versions of Eq. (3.64).

(6) Construct the Fourier amplitudes

$$
\begin{aligned}
& \tilde{s}_{1}\left(f_{i}\right):=\tilde{h}_{1}\left(f_{i}\right)+\tilde{n}_{1}\left(f_{i}\right), \\
& \tilde{s}_{2}\left(f_{i}\right):=\tilde{h}_{2}\left(f_{i}\right)+\tilde{n}_{2}\left(f_{i}\right) .
\end{aligned}
$$

(7) Whiten the data in the frequency domain by multiplying $\tilde{s}_{1}\left(f_{i}\right)$ and $\tilde{s}_{2}\left(f_{i}\right)$ by the frequency components $\widetilde{W}_{1}\left(f_{i}\right)$ and $\widetilde{W}_{2}\left(f_{i}\right)$ of the whitening filters of the two detectors:

$$
\begin{aligned}
& \widetilde{o}_{1}\left(f_{i}\right):=\widetilde{s}_{1}\left(f_{i}\right) \widetilde{W}_{1}\left(f_{i}\right), \\
& \widetilde{o}_{2}\left(f_{i}\right):=\widetilde{s}_{2}\left(f_{i}\right) \widetilde{W}_{2}\left(f_{i}\right) .
\end{aligned}
$$

This multiplication in the frequency domain corresponds to the convolution of $s_{1}\left(t_{i}\right)$ and $W_{1}\left(t_{i}\right) \quad\left[s_{2}\left(t_{i}\right)\right.$ and $\left.W_{2}\left(t_{i}\right)\right]$ in the time domain. (Note that the purpose of whitening the data is to reduce the dynamic range of the corresponding power spectra.)

(8) FFT $\widetilde{o}_{1}\left(f_{i}\right)$ and $\widetilde{o}_{2}\left(f_{i}\right)$ into the time domain to obtain the corresponding time series $o_{1}\left(t_{i}\right)$ and $o_{2}\left(t_{i}\right)$. (Here $t_{i}$ $:=i \Delta t$ where $i=0,1, \ldots, N-1$.)

(9) Repeat steps (4)-(8) to obtain another set of timeseries data $o_{1}\left(t_{i}\right)$ and $o_{2}\left(t_{i}\right)$. Distinguish these two different sets of data with superscripts: ${ }^{(1)} o_{1}\left(t_{i}\right),{ }^{(1)} o_{2}\left(t_{i}\right)$, ${ }^{(2)} o_{1}\left(t_{i}\right),{ }^{(2)} o_{2}\left(t_{i}\right)$.

(10) Offset ${ }^{(1)} o_{1}\left(t_{i}\right)$ and ${ }^{(2)} o_{1}\left(t_{i}\right)\left[{ }^{(1)} o_{2}\left(t_{i}\right)\right.$ and $\left.{ }^{(2)} o_{2}\left(t_{i}\right)\right]$ by $T / 2$, and combine them with one another and with data left over from the previous observation period to produce a continuous-in-time data set $o_{1}\left(t_{i}\right)\left[o_{2}\left(t_{i}\right)\right]$. $o_{1}\left(t_{i}\right)$ and $o_{2}\left(t_{i}\right)$ represent the "raw" (i.e., whitened) signal+ noise data streams output by the two detectors. (Note that steps (4)-(10) make up the signal generation part of the simulation.)

(11) Test the input data $o_{1}\left(t_{i}\right)$ and $o_{2}\left(t_{i}\right)$ to see if they have probability distributions consistent with that of a Gaussian random variable. If either set fails this test, reject them both, and repeat steps (4)-(10) to obtain new input data $o_{1}\left(t_{i}\right)$ and $o_{2}\left(t_{i}\right)$.

(12) Window the data streams $o_{1}\left(t_{i}\right)$ and $o_{2}\left(t_{i}\right)$ in the time domain, using a Hann window function to reduce sidelobe contamination of the corresponding power spectra.

(13) FFT the windowed data into the frequency domain to obtain the corresponding Fourier amplitudes $\tilde{o}_{1}\left(f_{i}\right)$ and $\tilde{o}_{2}\left(f_{i}\right)$.

(14) Unwhiten the data in the frequency domain by dividing $\widetilde{o}_{1}\left(f_{i}\right)$ and $\tilde{o}_{2}\left(f_{i}\right)$ by the frequency components $\widetilde{W}_{1}\left(f_{i}\right)$ and $\widetilde{W}_{2}\left(f_{i}\right)$ of the whitening filters of the two detectors:

$$
\begin{aligned}
& \tilde{s}_{1}\left(f_{i}\right):=\frac{\widetilde{o}_{1}\left(f_{i}\right)}{\widetilde{W}_{1}\left(f_{i}\right)}, \\
& \tilde{s}_{2}\left(f_{i}\right):=\frac{\widetilde{o}_{2}\left(f_{i}\right)}{\widetilde{W}_{2}\left(f_{i}\right)} .
\end{aligned}
$$

(15) Shift the input data streams $o_{1}\left(t_{i}\right)$ and $o_{2}\left(t_{i}\right)$ forward in time by $T / 2$, and repeats steps $(12)-(14)$, obtaining another set of Fourier components $\tilde{s}_{1}\left(f_{i}\right)$ and $\tilde{s}_{2}\left(f_{i}\right)$. Distinguish these two different sets of data with superscripts: ${ }^{(1)} \widetilde{s}_{1}\left(f_{i}\right),{ }^{(1)} \widetilde{s}_{2}\left(f_{i}\right),{ }^{(2)} \widetilde{s}_{1}\left(f_{i}\right),{ }^{(2)} \widetilde{s}_{2}\left(f_{i}\right)$.

(16) Average ${ }^{(1)} \tilde{s}_{1}\left(f_{i}\right)$ and ${ }^{(2)} \tilde{s}_{1}\left(f_{i}\right)\left[{ }^{(1)} \tilde{s}_{2}\left(f_{i}\right)\right.$ and

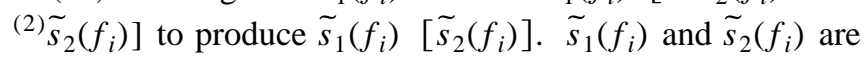
the Fourier components of the unwhitened time-series data $s_{1}\left(t_{i}\right)$ and $s_{2}\left(t_{i}\right)$. [Note that the purpose of this averaging is to reduce the variance in the estimation of the spectra $\tilde{s}_{1}\left(f_{i}\right)$ and $\left.\tilde{s}_{2}\left(f_{i}\right)\right]$.

(17) Construct the optimal filter function $\widetilde{Q}\left(f_{i}\right)$ with the overall normalization constant $\lambda$ chosen so that $\mu=\Omega_{0} T$, using the noise power spectra specified by the input data files.

(18) From $\tilde{s}_{1}\left(f_{i}\right), \tilde{s}_{2}\left(f_{i}\right)$, and $\widetilde{Q}\left(f_{i}\right)$ calculate the optimally filtered cross-correlation signal $S$ corresponding to a single observation period $T$. [Note that steps (11)-(18) make up the signal analysis part of the simulation.]

(19) Repeat steps (4)-(18) $n$ times, generating a set of optimally filtered cross-correlation signal values: $S_{1}, S_{2}, \ldots, S_{n}$.

(20) From $S_{1}, S_{2}, \ldots, S_{n}$ construct the sample mean

$$
\hat{\mu}:=\frac{1}{n} \sum_{i=1}^{n} S_{i}
$$

and sample variance

$$
\hat{\sigma}^{2}:=\frac{1}{n-1} \sum_{i=1}^{n}\left(S_{i}-\hat{\mu}\right)^{2} .
$$

The sample (or "experimental") signal-to-noise ratio produced by the simulation is given by 


$$
\widehat{\mathrm{SNR}}:=\frac{\hat{\mu}}{\hat{\sigma}} \text {. }
$$

(21) Calculate the theoretical signal-to-noise ratio, using a discrete frequency approximation to the integral

$$
\mathrm{SNR}=\Omega_{0} \frac{3 H_{0}^{2}}{10 \pi^{2}} \sqrt{T}\left[2 \int_{0}^{f_{\text {Nyquist }}} d f \frac{\gamma^{2}(f)}{f^{6} P_{1}(f) P_{2}(f)}\right]^{1 / 2} .
$$

See Eq. (3.75). [Note that since the data are discretely sampled, we should only integrate up to the Nyquist critical frequency $f_{\text {Nyquist }}:=1 /(2 \Delta t)$.]

(22) From $\widehat{\text { SNR }}$ and SNR, calculate the relative error

$$
\text { relative error }:=\left|\frac{\widehat{\mathrm{SNR}}-\mathrm{SNR}}{\mathrm{SNR}}\right| \text {. }
$$

As mentioned in Sec. VII A, this should be compared with the inverse of the theoretical signal-to-noise ratio after $n$ observation periods $1 /(\sqrt{n}$ SNR $)$. [Note that steps (20)-(22) make up the statistical analysis part of the simulation.]

Note that in order to obtain signal-to-noise ratios on the order of 10 after $n=1600$ runs, we needed to use rather large values of $\Omega_{0}$ (e.g., $10^{-3}$ for the initial LIGO detectors and $10^{-8}$ for the advanced LIGO detectors). These large values meant that expression (7.20) for the theoretical signalto-noise ratio had to be modified to properly take into account the contributions to the theoretical variance $\sigma^{2}$ that are due to a large stochastic gravity-wave signal. [See Eqs. (5.3) and (5.4).] Without these modifications, the theoretical and experimental signal-to-noise ratios would be more likely to disagree. Thus, instead of Eq. (7.20), we used a "mixed" expression for the theoretical signal-to-noise ratio:

$$
\mathrm{SNR}=\Omega_{0} \frac{3 H_{0}^{2}}{10 \pi^{2}} \sqrt{T} \frac{\sqrt{2} \int_{0}^{f_{\text {Nyquist }}} d f \frac{\gamma^{2}(f)}{f^{6} P_{1}(f) P_{2}(f)}}{\left[\int_{0}^{f_{\text {Nyquist }}} d f \frac{\gamma^{2}(f)}{f^{6} P_{1}^{2}(f) P_{2}^{2}(f)} R(f)\right]^{1 / 2}} .
$$

This involves Eqs. (5.3) and (5.4) for the variance $\sigma^{2}$, but the large noise expression (3.73) for the optimal filter function $\widetilde{Q}(f)$.

\section{CONCLUSION}

In this paper, we derived the optimal signal processing strategy required for stochastic background searches. We discussed signal detection, parameter estimation, and sensitivity levels from a frequentist point of view. We also discussed the complications that arise when one considers (i) arbitrarily large stochastic backgrounds, (ii) non-stationary detector noise, (iii) multiple detector pairs, and (iv) correlated detector noise. We explained how we verified some of the theoretical calculations by writing a computer simulation that mimics the generation and detection of a simulated stochastic gravity-wave signal in the presence of simulated detector noise. And we noted that the "experimental" results and theoretical predictions agreed to within the expected error.
These results suggest that both the theoretical signal processing formulas and the implementation of these formulas in a computer code are correct. But we should not stop here. For example, there are still a number of ways that we can improve the data analysis code before we use it to search for a real stochastic background in the outputs of real interferometers. To conclude this paper, we list some of the desired improvements below:

(i) The first change that we would like to make is to calculate real-time noise power spectra for the detectors, and to use this calculated data (rather than the information contained in the input noise power spectrum data files) to construct the optimal filter function $\widetilde{Q}\left(f_{i}\right)$. [See step (17) in the computer simulation described in Sec. VII B.] Since the realtime noise power spectra will change slightly from one measurement to the next, we could then apply the data analysis strategy discussed in Sec. V B for nonstationary detector noise.

(ii) In order to obtain accurate real-time noise power spectra for the two detectors, it will probably be necessary to use more sophisticated spectral estimation techniques. Currently, we use a Hann window to reduce side-lobe contamination, and we average two overlapped data sets to reduce the variance, when forming our estimates of $\tilde{s}_{1}\left(f_{i}\right)$ and $\tilde{s}_{2}\left(f_{i}\right)$. [See steps (12), (15), and (16) of the computer simulation.] This procedure can be replaced by multitaper spectral estimation methods, which use a special set of window functionscalled Slepian tapers- to form spectral estimates of timeseries data. ${ }^{43}$ GRASP [27] contains a modified version of a public domain package by Lees and Park [30] to perform the multitaper spectral estimation. In addition to providing better spectral estimates, multitaper methods also provide nice techniques for "spectral line" parameter estimation and removal. This feature will be extremely useful when analyzing data produced by a real detector. For example, one will be able to track contamination of a data set by the line harmonic at $300 \mathrm{~Hz}$, and remove a pendulum resonance at, say, 590 Hz. (See GRASP [27] for more information.)

(iii) In addition to being able to identify and to remove "spectral lines" from a real data set, one would also like to be able to test the data to see if the distribution of sampled values is consistent with normal detector operation. For example, one might check the input data set to see if it has a probability distribution consistent with that of a Gaussian random variable. If the test reveals an exceptionally large number of "outlier" points, then that particular data set can be rejected. [See step (11) of the computer simulation.] The GRASP data analysis package already contains a routine that performs this Gaussian test. But we would also like a more rigorously characterized test that compares the distribution of the current data with that during "normal" detector operation, which most likely is not Gaussian.

(iv) Finally, even though it will still be a few years before we can analyze real data from any one of the major interfer-

\footnotetext{
${ }^{43}$ See the original paper by Thomson [28] and the text by Percival and Walden [29] for more details.
} 
ometers, real data from prototypes-like the Caltech 40-m interferometer-can be used in computer simulations. For instance, rather than write a computer simulation (like the one we described in Sec. VII) that mimics the generation and detection of a simulated stochastic gravity-wave signal in the presence of simulated detector noise, we can write a computer simulation that mimics the generation and detection of a simulated stochastic gravity-wave signal in the presence of real detector noise. ${ }^{44}$ The fact that the noise level of a prototype interferometer is much larger than that of a major interferometer poses no problem; we can simply "dial-in" a larger stochastic background signal to be able to detect it in the same amount of observation time. Another nice feature of this fake stochastic background and real detector noise simulation is that we can address all of the issues (i)-(iii) discussed above in a context where we can still compare "experimental" (i.e., simulation) performance against theoretical expectations. We must be totally convinced that the

\footnotetext{
${ }^{44}$ The real detector noise would be provided by the prototype output.
}

data analysis code is working as expected, before we can trust it when searching for a real stochastic background in the outputs of real interferometers.

\section{ACKNOWLEDGMENTS}

This work has been partially supported by NSF grant PHY95-07740 to the University of Wisconsin at Milwaukee and NSF grant PHY93-08728 to Northwestern University. We would like to thank Luca Gammaitoni, Albrecht Rudiger, Kenneth Strain, Masa-Katsu Fujimoto, and Rainer Weiss for kindly supplying the numerical data for the noise power spectra for the VIRGO, GEO-600, TAMA-300, and "enhanced" LIGO detectors. We would also like to thank Sam Finn for carefully proofreading Sec. IV, and for explaining the differences between the Bayesian and frequentist approaches to data analysis, and Eric Key for bringing the law of the iterated logarithm to our attention. B.A. gratefully acknowledges the LIGO visitors program for support under NSF grant PHY96-03177, and useful conversations with Kent Blackburn, Ron Dreever, Eanna Flanagan, B.S. Sathyaprakash, David Shoemaker, Kip Thorne, Robbie Vogt, Rainer Weiss, and Stan Whitcomb.
[1] A. Abramovici et al., Science 256, 325 (1992).

[2] B. Caron et al., in Gravitational Wave Experiments, Proceedings of the Edoardo Amaldi Conference, edited by E. Cocia, G. Pizzella, and F. Ronga (World Scientific, Singapore, 1995), p. 86.

[3] K. Danzmann et al., in Gravitational Wave Experiments [2], p. 100.

[4] K. Tsubono, in Gravitational Wave Experiments [2], p. 112.

[5] P.F. Michelson, Mon. Not. R. Astron. Soc. 227, 933 (1987).

[6] N. Christensen, Phys. Rev. D 46, 5250 (1992).

[7] E. Flanagan, Phys. Rev. D 48, 2389 (1993).

[8] B. Allen, in Proceedings of the Les Houches School on Astrophysical Sources of Gravitational Waves, edited by J.A. Marck and J.P. Lasota (Cambridge University Press, Cambridge, England, 1997), p. 373.

[9] E.W. Kolb and M. Turner, The Early Universe, Frontiers in Physics (Addison-Wesley, Reading, MA, 1990).

[10] B. Allen and A.C. Ottewill, Phys. Rev. D 56, 545 (1997).

[11] D.G. Blair, R. Burman, L. Ju, S. Woodings, M. Mulder, and M.G. Zadnik, "The supernova cosmological background of gravitational waves," report, University of Western Australia, 1997; V. Ferraria, in Proceedings of the XII Italian Conference on GR and Gravitational Physics, edited by M. Bassan et al. (World Scientific, Singapore, 1997); D.G. Blair and L. Ju, Mon. Not. R. Astron. Soc. 283, 648 (1996).

[12] C.W. Misner, K.S. Thorne, and J.A. Wheeeler, Gravitation (Freeman, San Francisco, 1973).

[13] G.F. Smoot et al., Astrophys. J. Lett. 396, L1 (1992).

[14] C.L. Bennett et al., Astrophys. J. Lett. 396, L7 (1992).

[15] C.L. Bennett et al., Astrophys. J. 436, 423 (1994).

[16] C.L. Bennett et al., Astrophys. J. Lett. 464, L1 (1996).
[17] V. Kaspi, J. Taylor, and M. Ryba, Astrophys. J. 428, 713 (1994).

[18] L.S. Finn, "Gravitational-wave data analysis with multiple detectors: The gravitational-wave receiver. I. Deterministic sources"' (in preparation).

[19] L.S. Finn, "Gravitational-wave data analysis with multiple detectors: The gravitational-wave receiver. II. Stochastic signals" (in preparation).

[20] I. Miller and J.E. Freund, Probability and Statistics for Engineers (Prentice-Hall, Englewood Cliffs, NJ, 1985).

[21] W.H. Beyer, CRC Standard Probability and Statistics Tables and Formulae (CRC, Boca Raton, 1991).

[22] C.W. Helstrom, Statistical Theory of Signal Detection, 2nd ed. (Pergamon, Oxford, 1968).

[23] W. Feller, An Introduction to Probability Theory and Its Applications (Wiley, New York, 1950), Vol. 1.

[24] N. Christensen, Ph.D. thesis, Massachusetts Institute of Technology, 1990.

[25] D. Coyne, LIGO project (private communication).

[26] The data for the predicted noise power spectra for the initial and advanced LIGO detectors were taken from [1]. Those for the VIRGO detector were supplied by Luca Gammaitoni (E-mail address: gammaitoni@perugia.infn.it); the GEO-600 detector by Albrecht Rudiger and Kenneth Strain (E-mail address: atr@mpq.mpg.de and kstrain@physics.gla.ac.uk); and the TAMA-300 detector by Masa-Katsu Fujimoto (E-mail address: fujimoto@gravity.mtk.nao.ac.jp). The predicted noise power spectra for the "enhanced" LIGO detectors were supplied by Rainer Weiss (E-mail address: weiss@tristan.mit.edu).

[27] B. Allen, "GRASP: a data analysis package for gravitational wave detection," 1997. An up-to-date 
version of the users manual may be obtained at http://www.ligo.caltech.edu/LIGO_web/Collaboration/ manual.pdf or http://www.ligo.caltech.edu/LIGO_web/ Collaboration/lsc_interm.html. The software package is available upon request.

[28] D.J. Thomson, Proc. IEEE 70, 1055 (1982).
[29] D.B Percival and A.T. Walden, Spectral Analysis for Physical Applications (Cambridge University Press, Cambridge, England, 1993).

[30] J.M. Lees and J. Park, Compu. Geol. 21, 199 (1995). The multitaper spectral estimation public domain package can be found at the website http://love.geology.yale.edu/mtm/. 